\title{
Cooperative Signal Amplification for Molecular Communication in Nanonetworks
}

Author: Sergi Abadal Cavallé

Advisors: Albert Cabellos Aparicio and Ignacio Llatser Martí

September 7, 2011 

NaNoNetworking Center in Catalunya, Universitat Politècnica de Catalunya, Barcelona February, 2011 - June, 2011 


\begin{abstract}
Nanotechnology is enabling the development of devices in a scale ranging from a few to hundreds of nanometers. Communication between these devices greatly expands the possible applications, increasing the complexity and range of operation of the system. In particular, the resulting nanocommunication networks (or nanonetworks) show great potential for applications in the biomedical field, in which diffusion-based molecular communication is regarded as a promising alternative to electromagnetic-based solutions due to the bio-stability and energy-related requirements of this scenario. In this new paradigm, the information is encoded into pulses of molecules that reach the receiver by means of diffusion. However, molecular signals suffer a significant amount of attenuation as they propagate through the medium, thus limiting the transmission range.

In this work we propose, among others, a signal amplification scheme for molecular communication nanonetworks in which a group of emitters jointly transmits a given signal after achieving synchronization by means of Quorum Sensing. By using the proposed methodology, the transmission range is extended proportionally to the number of synchronized emitters. We also provide an analytical model of Quorum Sensing, validated through simulation. This model accounts for the activation threshold (which will eventually determine the resulting amplification level) and the delay of the synchronization process.
\end{abstract}





\section{Contents}

1 Introduction 5

1.1 Nanotechnology . . . . . . . . . . . . . . . . . . . . 5 5

1.2 Nanomachines . . . . . . . . . . . . . . . . . . . . . 6

1.3 Assembling Approaches . . . . . . . . . . . . . . . 7

1.4 Interaction Between Nanomachines . . . . . . . . . . . . . . . . . 10

1.4.1 Molecular Communication . . . . . . . . . . . . . . . 11

1.4.1.1 Diffusion-based Molecular Communication . . . . . . . . 15

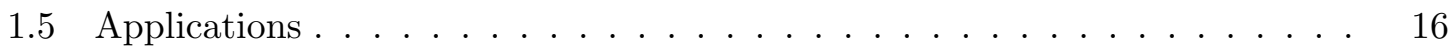

2 Motivation $\quad 18$

3 Quorum Sensing $\quad 21$

3.1 Principles and Mechanisms _. . . . . . . . . . . . . 22

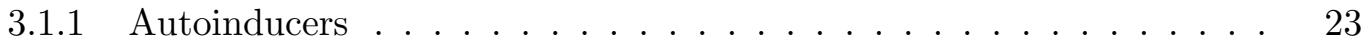

3.1 .2 Thresholds . . . . . . . . . . . . . . . . . . 23

3.2 Types of Quorum Sensing Systems _. . . . . . . . . . . . . 25

3.3 Combination of Quorum Sensing Systems . . . . . . . . . . . 26

3.4 Bacterial Behaviors Controlled by Quorum Sensing . . . . . . . . . . . . 28

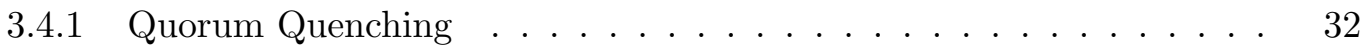

3.5 Communication Aspects of Quorum Sensing . . . . . . . . . . . . 33

3.6 Applications of Quorum Sensing . . . . . . . . . . . . . 34 
4 Framework $\quad 43$

4.1 Analytical Environment . . . . . . . . . . . . . . . . . 43

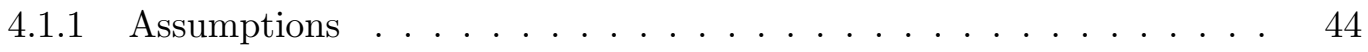

4.1 .2 Diffusion of Autoinducers . . . . . . . . . . . . . . . 47

4.1.2.1 Fick's Laws of Diffusion . . . . . . . . . . . . . 48

4.1.2.2 Finite Differences Method Applied to Fick's Laws . . . . . 50

4.2 Simulated Environment f . . . . . . . . . . . . . . . . 51

4.2 .1 Existing Simulators _. . . . . . . . . . . . . 51

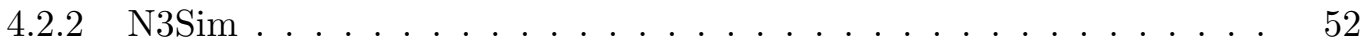

5 Analytical Model and its Validation $\quad 61$

5.1 Individual Emission f . . . . . . . . . . . . . . . . . . . . 62

5.2 Autoinducers Accumulation . . . . . . . . . . . . . . . 64

5.3 Threshold Calculation . . . . . . . . . . . . . . . . . . . 69

5.4 Delay Calculation . . . . . . . . . . . . . . . . . . . 71

6 Amplification $\quad 75$

6.1 Transmission Range . . . . . . . . . . . . . . . . 77

6.2 Threshold Election . . . . . . . . . . . . . . . . . . . . 79

7 Open Issues and Conclusions $\quad 81$

7.1 Open Issues . . . . . . . . . . . . . . . . . . . . . . . . 81

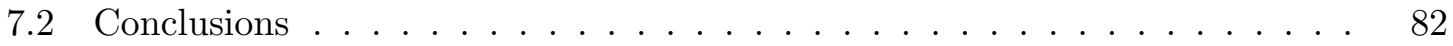

$\begin{array}{ll}\text { A Existing Models of Quorum Sensing } & 84\end{array}$ 



\section{Chapter 1}

\section{Introduction}

In December 1959, the future Nobel laureate physicist Richard Phillips Feynman gave a lecture at Caltech with the title "There's Plenty of Room at the Bottom". In his speech, he envisaged the miniaturization of devices down to atomic levels. He proposed ways to accomplish that miniaturization, and predicted that the key of future technologies will lie in the arrangement of individual atoms. This was the first time that concepts related to nanotechnology were pointed out.

At that time, Dr. Feynman could not have imagined that more than 50 years later, some of his ideas remain up-to-date, and that also a lot of research and major accomplishments have been achieved in this field in the direction that he envisaged.

\section{$1.1 \quad$ Nanotechnology}

The term "Nanotechnology" was not defined until 1974. Professor Norio Taniguchi coined it by stating: "Nanotechnology mainly consists of the processing of, separation, consolidation, and deformation of materials by one atom or by one molecule" [75].

However, it was not until years later that feasible advancements in nanotechnology arose, because of how revolutionary the ideas of doctor Feynman were. In 1981, the Scanning Tunneling Microscope (STM) was invented, which was capable to image surfaces at the atomic level. This led to the discovery of carbon structures in the late 1980s, such as carbon nanotubes (CNTs), which showed novel properties potentially useful in many areas. 
Following these discoveries, the activity surrounding the nanotechnology advanced slowly. The early 2000s marked the beginning of a period in which major steps have been taken and in which the popularity has increased immensely.

Nanotechnology encompasses the development of structures and applications involving control of matter on an atomic and molecular scale, ranging typically from 0.1 to 100 nanometers. One nanometer $(\mathrm{nm})$ is one billionth $\left(10^{-9}\right)$ of a meter; and by comparison, about a 1/100000 of the diameter of a human hair, or, on the other hand, half of the diameter of a DNA double-helix.

It is at this scale where the classical approximations and assumptions of Newton physics are not valid anymore, and quantum physics come into play. Nanotechnology studies the new conditions imposed by quantum physics, and the new chemical and physical properties of the particles at the nanoscale. This insight will help understand the behavior of devices lying in the nanoscale and the way they interact with their close environment.

Nanotechnology provides new solutions for applications in a wide range of fields. One of the reasons is that nanotechnology was conceived as a multidisciplinary discipline, comprising diverse areas of study such as chemistry, physics, molecular biology, computer science and, in our case, telecommunications.

\subsection{Nanomachines}

One of the main objectives of nanotechnology is to successfully arrange nanomachines. Nanomachines are "artificial or biological nanoscale devices that perform simple computation, sensing, or actuation" [72]. These devices are usually regarded as the most basic functional unit at this scale, and can be used as building blocks in order to construct more complex systems [3].

These new and more complex systems, may not be strictly nano in size, but keep performing their tasks in the nanoscale, and taking advantage of the unique properties of nanomaterials or nanoparticles (e.g. quantum physics) to serve its purpose. Nanosensors [4] and nanoswitches $[15,33]$ are good examples of this. Nanosensors are capable of detecting chemical compounds in almost an atomic resolution, whereas nanoswitches are envisaged to 
perform optical switching through certain molecules, dramatically reducing the switching delay.

Nanomachines count on size as the most identifying feature. Consequently, other properties or limitations can be deduced from it.

- First of all, low complexity is expected, owing to the fact that manufacturing extremely complex systems in the nanoscale is not feasible so far.

- Because of size and lack of complexity, low consumption is to be a desired feature, since most of the nanomachines' applications will require the use of autonomous power sources.

- Another expected characteristic of nanomachines is low range, since the aforementioned properties (e.g. size and low complexity) restrict the area of action to its very close environment.

- Biocompatibility is an expected feature of nanomachines since the nanoscale is the natural domain of molecules, proteins and the major components of cells [5].

- Last but not least, sensitivity. The fact that a nanomachine can be formed by only several atoms, makes it highly sensible to even the lightest perturbations. As an example, the adsorption of single molecules in a nanoantenna surface can change its radiation properties [4].

\subsection{Assembling Approaches}

In order to manufacture and assemble nanomachines, three different techniques have been stated, namely, top-down, bottom-up and bio-hybrid approaches.

\section{Top-down Approach}

The top-down approach is the most intuitive approach if we follow the miniaturization tendency in electronics (i.e. Moore's Law). It was first pointed out by Richard Feynman, who 


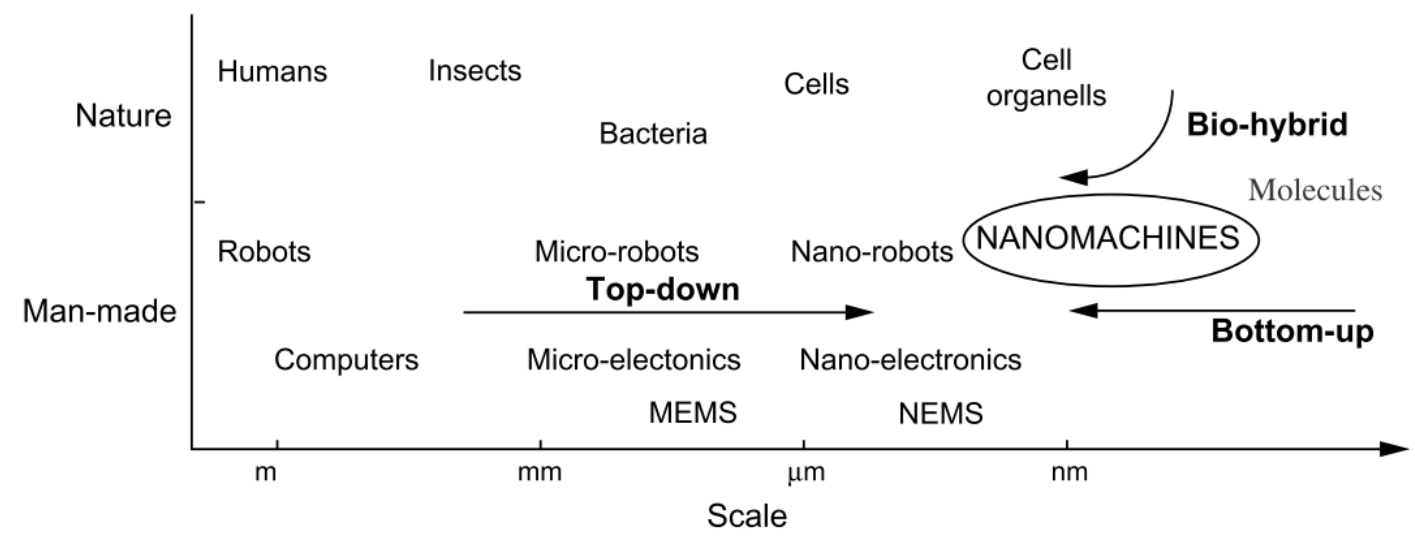

Figure 1.1: Approaches for the development of nanomachines.

stated that further miniaturization would be possible in order to write and read information in an atomic scale.

Hence, in this approach, nanomachines are developed by means of downscaling current microelectronic and micro-electro-mechanical (MEMS) devices, conserving its architecture, further to the nanoscale $[29,49]$. The evolution of classical lithography techniques enabled the manufacturing of components in dimensions below $100 \mathrm{~nm}$. Furthermore, new nanofabrication techniques as microcontact printing [44], imprint lithography [13] and direct-write dip-pen nanolithography [67] have been developed and are currently used.

It was in 2000, that Intel started the large-scale production of transistors with lengths below 100 nanometers, using these new techniques. In fact, CMOS transistors measuring $45 \mathrm{~nm}$ or even $32 \mathrm{~nm}$ long have been developed, and it has been envisaged that 22, 16 and 11 nanometer transistors will be manufactured in the near future.

\section{Bottom-up Approach}

In the bottom-up approach, nanomachines are constructed using individual molecules as building blocks, in a process called molecular manufacturing [23]. Or, generally speaking, smaller components are use to build up more complex assemblies [4].

An example of an existing type of manufacturing based in this approach is the assembly by DNA scaffolding. In this technique, structures of synthesized DNA known as "DNA 
origami" are arranged in a silicon surface, serving as a mold to assemble different nanoscale components particle by particle [63]. DNA sequences are composed by series of nucleotides, namely, adenine, guanine, thymine and cytosine, which are complementary two by two. The particles to assemble have DNA strands complementary to the strands present in the scaffold. This way, they can only be connected to the silicon surface in a single position with a defined orientation.

The synthesizing of DNA strands is programmable, making this technique suitable for nanomachine manufacturing. Furthermore, it has been shown that the use of DNA origami makes the placement of atoms or molecules $100 \%$ accurate $[7,32]$.

\section{Bio-hybrid Approach}

In this approach, also known as bio-inspired or biomimetic, biological components are proposed as models for new nanomachines, or even used as building blocks, integrating them into more complex systems [3]. Hence, it involves the understanding, conceptualization and/or mimicking nature's ways of handling various problems or situations.

Often, inside cells, we can find lots of biological structures that carry out functions such as sensing, actuation, storing, control [22], executed in a highly effective and energy efficient ways. Moreover, these natural nanomachines show some properties that would be desired for future nanomachines:

- Self-continence: usually cells have their set of instructions completely specified in the DNA of its nucleus or nucleoid.

- Self-replication: is the property through which nanomachines autonomously replicate themselves and its set of instructions.

- Self-assembly: is defined as the process in which several disordered elements form an organized structure without external intervention, as a result of local interactions between them.

An example of them is stated when the bottom-up approach was explained. DNA scaffolding creates new nanomachines helped by the self-assembly property, owing to the fact that the binding of complementary DNA strands is a non externally-driven process.

Cooperative Signal Amplification for Molecular Communication in Nanonetworks 
Some other systems found in nature would enable useful features in nanomachines. For instance, locomotion can be accomplished by means of using different organelles such as flagellum, present in several bacteria like E. Coli. Communication capabilities are also envisaged to be important, and different examples of natural components that enable this characteristic can be found in Section 1.4 (e.g. molecular motors or communication through flagellated bacteria).

In summary, the bio-hybrid approach is considered the most promising of all assembling techniques, because the complexity of its components and the good properties that intrinsically are contained in them. Bottom-up also shows great promise, due to the fact that each atom can be arranged at will. On the other hand, the top-down approach offers great possibilities nowadays, but the potential of the other two options seems that will pave the best way for nanodevice manufacturing and assembly.

\subsection{Interaction Between Nanomachines}

The transmission range of nanodevices is extremely limited due to their reduced size and energy constraints. Communication between these nanodevices greatly enhances and expands the capabilities of single nanodevices, increasing the complexity and range of operation of the system [3]. Therefore, networks of nanomachines (otherly referred as nanonetworks) can be used to coordinate tasks and realize them in a distributed manner, covering a greater area and reaching unprecedented locations.

How nanomachines will communicate is still an important research challenge. Traditional wireless electromagnetic communication, by means of graphene-based nano-antennas, has been proposed to address this issue $[6,39,66]$. These techniques are expected to produce ultra-high frequency radiation (in the $\mathrm{THz}$ range [38]), offering low propagation delays and high bandwidth. However, biomedical applications (and particularly intra-body networks) usually require the use of biocompatible and non-invasive solutions. While the biocompatibility of EM-based techniques remains under study, their energy efficiency figures render impractical their use in such an energy-constraint scenario. These issues therefore compromise the suitability of electromagnetic communication in the biomedical scenario.

Cooperative Signal Amplification for Molecular Communication in Nanonetworks 


\subsubsection{Molecular Communication}

Molecular communication is a novel and promising way to achieve communication between nanodevices by encoding messages inside molecules. Three processes appear in this new paradigm: emission, propagation, and reception. First, emitters release molecules as a response to a certain command. These particles propagate through the medium either by following pre-defined pathways, guided diffusion flows, or simply by means of spontaneous diffusion. On the receiver side, specific signal transducing mechanisms chemically react to concentrations of particles allowing the receiver to decode the message. Figure 1.2 shows the processes that are present in diffusion-based molecular communication [61], which will be depicted in Section 1.4.1.1.

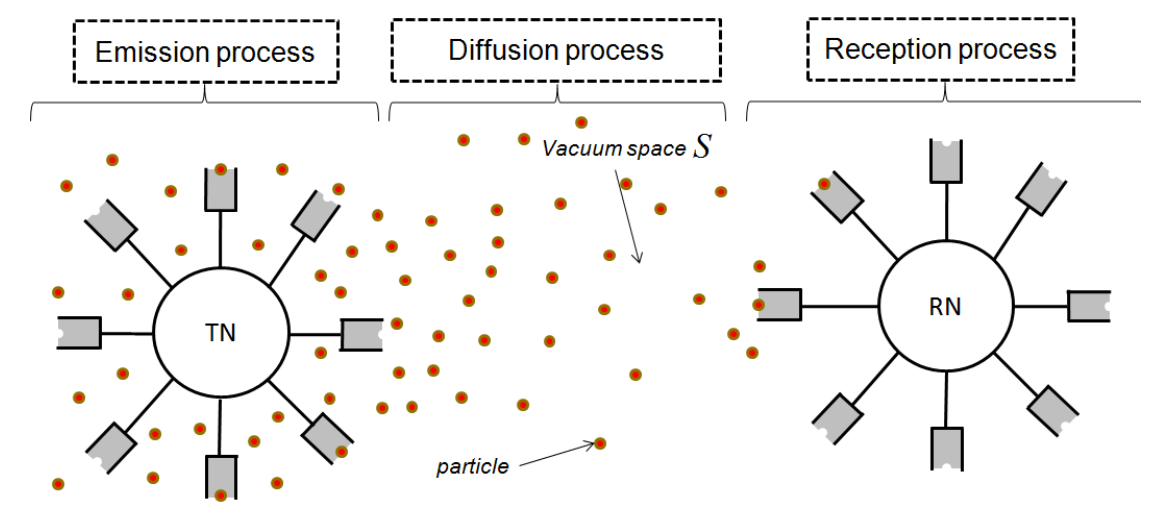

Figure 1.2: The three steps present in diffusion-based molecular communication.

Indeed, the molecular communication paradigm mimicks communication techniques developed by nature. It is broadly considered as the most promising option, as it features three significant advantages:

- Size: nanomachines have an obvious limitation in space, rendering the embedding of traditional transceivers impractical. On the contrary, molecular transducers inherently lie in the nanoscale and are expected to be easy to integrate.

- Biocompatibility: in some applications, the use of devices and systems that will not affect the environment is needed (e.g. intra-body networks). The encoding of messages in molecules and the presence of biological transducing mechanisms ensures the interaction with natural processes without any side effect. 
- Energy consumption: nanomachines should maintain its consumption low, as the harvesting of energy is an important challenge in this scenario. Chemical reactions present in the process of biological transceiving are extremely efficient in terms of energy.

\section{Range-based Classification of Molecular Communication}

Following these guidelines, several solutions for molecular communications have been proposed depending on the distance between emitter and receiver (see Figure 1.3). Different approaches have been taken in order to cover distances spanning from nanometers to tenths of meters [3].

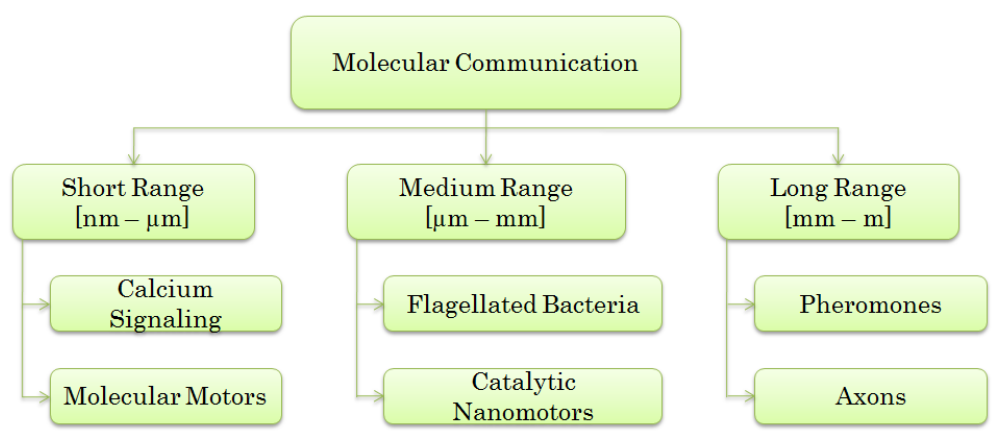

Figure 1.3: Molecular Communication Techniques.

Short range techniques allow communication in distances up to micrometers. Two of them stand out from the other possibilities, namely molecular signaling and molecular motors. The former consists of encoding the message in the concentration of molecules emitted. This concentration of molecules can be modulated both in frequency and amplitude [61], and after emitted, they will diffuse away following Brownian motion laws. A natural inspired example of this kind is the calcium signaling, in which the carrier is the concentration of calcium ions $\left(\mathrm{Ca}^{2+}\right)$ [55]. The latter option relies on the use of molecular motors, which are proteins or protein complexes that transform chemical energy into mechanical work. The message will be encoded in molecules, macromolecules, or a set of molecules encapsulated in a vesicle, which will be loaded to the motor, which will move directionally 
along cytoskeletal tracks. Well-known examples of molecular motors are dynein, kinesin and myosin [51].

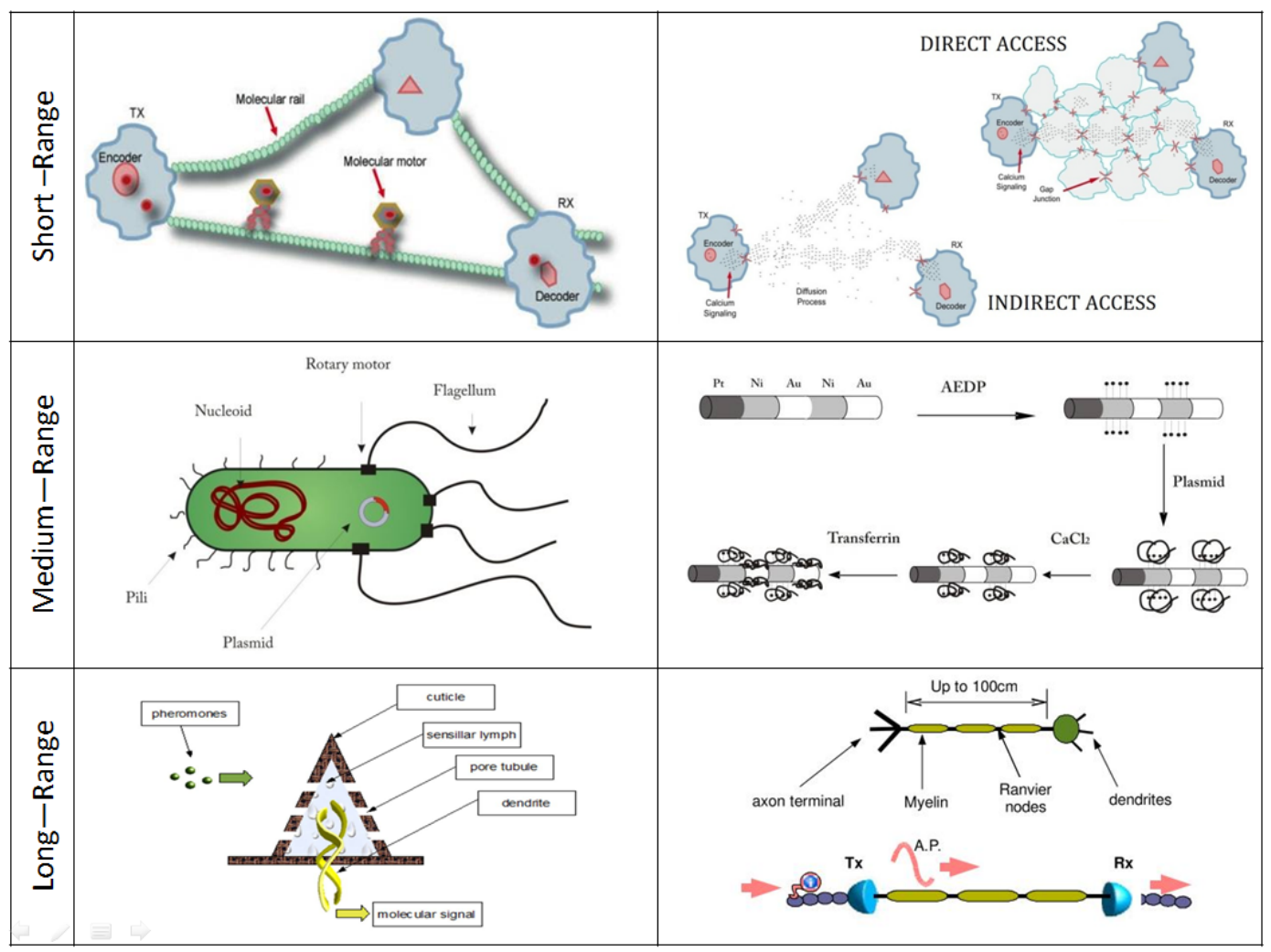

Figure 1.4: Molecular Communication schemes.

Medium range solutions arise after observing that short range techniques are greatly inefficient as the distance grow. Molecular motors move slowly along the tracks $(500 \mathrm{~nm}$ per second) and it has been observed that they detach after distances longer than $1 \mu \mathrm{m}$. In the case of molecular signaling, it is known that the diffusion time is a function of the square of the distance. Thus, at certain distances, the delay can be unacceptable. In [31], two mechanisms of medium range communication are proposed: Flagellated bacteria and catalytic nanomotors. Both of them encode the information in DNA sequences, and guide those to the receiver through a process based on a phenomenon called chemotaxis, by which the bacteria move following gradients of attractant particles. Both mechanisms show 
speeds tenths of times higher than short range techniques, as well as increased reliability at distances of several micrometers.

Finally, long range techniques stem from the necessity to communicate nanomachines separated more than $1 \mathrm{~mm}$. Both "wired" and "wireless" solutions have been proposed [57]. Wireless techniques, namely, pheromones, pollen, and spores do not need electrical conductors or other physical link but a fluid medium to propagate. Concretely, pheromones are diffusion-based while pollen and spores have to be transported by a flowing fluid. On the other hand, wired mechanisms rely on a physical infrastructure to propagate the message, like the emission and reception of hormones through a network of capillaries. The encoding of the message follows the same fashion as short-range techniques: information can be contained in the rate concentration of the molecules used, as well as in DNA packets embedded in each of them. The receptors count on a high selectivity, thus binding only to specific agents, particularly in the pheromone and hormone cases [43].

Further information and analysis of these three approaches of molecular communication, and their multiple options can be found in $[3,31,57]$ respectively.

\section{Architecture-based Classification of Molecular Communication}

As previously discussed, particles propagate through the medium following different mechanisms in what has been called "propagation process". Indeed, the characterization of how molecules propagate leads to the definition of three different nanonetwork architectures [61] (see Figure 1.5). The architecture is considered to be diffusion-based or flow-based when the propagation of molecules takes place in a fluidic medium by following spontaneous diffusion or a guided flow, respectively. Whereas in walkway-based architectures, particles propagate following pre-defined pathways that connect transmitter and receiver.

Hence, different molecular communication techniques can be classified depending on its architecture. For instance, communication through molecules carried by molecular motors [72] is a clear example of a walkway-based architecture. Nevertheless, the limited range of the solution and the need of a network infrastructure renders this option impractical. On the other hand, examples of flow-based architecture include hormonal communication through blood streams [61] or guided transport of information by means of bacterial communication 


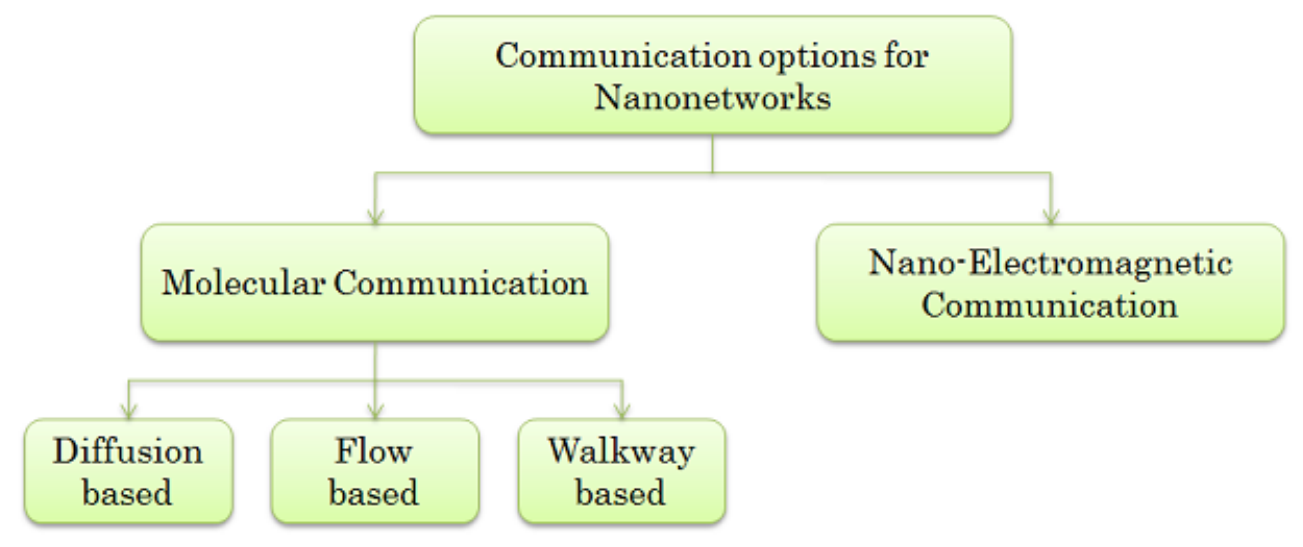

Figure 1.5: Alternative classification of communication options for nanonetworks.

$[31,14]$. Finally, diffusion-based mechanisms have been identified both for short-range and long-range nanonetworks, namely calcuim signalling [55] and pheromonal communication $[57]$.

\subsubsection{Diffusion-based Molecular Communication}

In this work we will focus on indirect molecular signaling among the presented types of molecular communication. This technique is classified as diffusion-based and, in principle, short-range solution.

We consider that the space where the communication takes place contains a fluidic medium with an homogeneous concentration of molecules [61]. Under these conditions, molecules released by the emitters propagate through the medium by means of spontaneous diffusion [72] until they reach the receiver. In this case, the molecules move following concentration gradients in a process that can be modeled using the Fick's laws of diffusion [58]. Figure 1.2 summarizes the different steps of the diffusion-based communication process.

In summary, molecular transmitters will transmit a message encoded in a variable concentration of communication molecules that will propagate towards the receiver by means of diffusion. Actually, experimental results led to the conclusion that cells can adopt modulation schemes similar to the traditional AM or FM techniques [56]. Since the complexity of nanomachines is expected to be very low, researchers are proposing simple modulation 
techniques, such as concentration-based ON-OFF modulations that encode information into pulses $[28,47]$. In this context the receiver interprets low and high molecular concentrations as "absence" or "presence" of a pulse, and thus decodes a bit accordingly. The interested reader can find more details about diffusion-based molecular nanonetworks in [62].

\subsection{Applications}

Nanonetworks expand the possible applications of nanomachines in an almost unlimited extent. Mainly, applications of networks of nanomachines can be classified in five big areas: biomedical, environmental, industrial, military, and ICT applications [3, 39]. Nevertheless, nanotechnology plays a fundamental role in the manufacturing of lots of devices nowadays, thus affecting fields other than the five main areas (e.g. consumer electronics or sportsrelated fields).

\section{Biomedical Applications}

The most direct applications of nanonetworks are considered to be in the biomedical field. In fact, it is probably the first area in which intuitively scientists think when talking about nanomachines' applications, because of nanomachines' size and their envisioned biocompatibility.

Concrete applications in this field are immune system support [12], bio-hybrid implants or tissue engineering [22], health monitoring mechanisms, drug delivery systems [25] and applications including genetic engineering.

\section{Environmental Applications}

Especially, bio-inspired nanodevices (such as nanofilters [34]) and nanonetworks can be applied in the environmental field to reach several goals unachievable with classical technologies.

Biodegradation purposes, animals and biodiversity control, and air pollution control are good examples of environmental applications.

Cooperative Signal Amplification for Molecular Communication in Nanonetworks 


\section{Industrial and Consumer Goods Applications}

Nanonetworks can help with the development of new materials, manufacturing processes and quality control procedures.

Examples of this kind of applications would be food and water quality control, or functionalized materials and fabrics (e.g. stain-repellent or antimicrobial [76] textiles, and easyto-clean surfaces or paints).

\section{Military Applications}

The range of operations of nanonetworks applied to military applications is really wide. Small scope applications could be used just to monitor soldiers' performance through nano-

functionalized equipments. On the other hand, nanonetworks covering broader areas can be deployed in order to monitor the battlefield, to detect chemicals or toxics, for instance. It receives the name of nuclear, biological and chemical (NBC) defenses.

\section{ICT Applications}

Information and Communication Technologies (ICT) will obviously take advantage of all the potential and new features that nanonetworks offer, as networking is one of the pillars of ICTs.

Examples of this kind of applications are: the integration of nano-sensor networks [70], distributed information access, and Future Internet.

Applications in which the work of this thesis can help improve performance are explained in more detail in Section 3.6. 


\section{Chapter 2}

\section{Motivation}

Nanotechnology in general, and nanonetworks in particular, are fields that have attracted a lot of attention the last years and this interest will do nothing but increase in the future. As telecommunication engineers, the focus of this study is on discovering new tools for the communication between nanodevices.

Molecular communication has been presented as an alternative to classical electromagnetic schemes, since some of the most promising applications of nanonetworks (in the biomedical field, for example) usually require the use of biocompatible and non-invasive solutions. While the biocompatibility of EM-based techniques remains under study, their energy efficiency figures render impractical their use in such an energy-constraint scenario. These issues therefore compromise the suitability of electromagnetic communication in the biomedical scenario.

In this work we focus in diffusion-based molecular communication, which is used by cells to communicate among them (see Section 1.4.1.1). Cells encode information into molecules that are released until they eventually reach the receiver, that is, the molecules are physically transported by means of diffusion to the receiver. This mechanism is based on completely different principles when compared to EM-based communications and requires developing radically new communication principles.

For instance, recent results on the characterization of the physical channel of diffusionbased molecular communication show that encoding the information to be transmitted into

pulses of molecules presents significant challenges [45]. Besides the addition of noise from 
Table 2.1: Scalability of communication metrics in wireless electromagnetic and molecular channels $[45]$

\begin{tabular}{l|c|c} 
Metric & EM channel & Molecular channel \\
\hline Pulse delay & $\Theta(r)$ & $\Theta\left(r^{2}\right)$ \\
Pulse amplitude & $\Theta\left(1 / r^{2}\right)$ & $\Theta\left(1 / r^{3}\right)$ \\
Pulse width & $\Theta(1)$ & $\Theta\left(r^{2}\right)$ \\
\multicolumn{2}{c}{$[r$ : transmission distance $]$}
\end{tabular}

several sources [60], these pulses suffer a great amount of attenuation, delay and distortion as they propagate through the medium (see Table 2.1). In particular, results show that the amplitude of a molecular pulse is inversely proportional to the third power of the transmission distance $r, \Theta\left(1 / r^{3}\right)$ [45]. Please note the difference with the scalability of classical EM techniques: $\Theta\left(1 / r^{2}\right)$. Ensuring the connectivity of the network under these conditions is an open challenge, as the range of individual nanomachines is strongly limited by these attenuation figures.

To exemplify the problem, we performed two simulations in which 2-millisecond long pulses, with a constant amplitude of 50000 particles, were transmitted. The receiver is located 10 micrometers away from the transmitter in the first case, whereas in the second simulation the receiver is situated at a distance of 25 micrometers. These simulations were realized using the N3Sim framework that will be introduced in Section 4.2.2.

Figure 2.1 shows the evolution over time of the particle concentration received in these two different cases. While the pulse received in the first case can be easily distinguished, the pulse in the second receiver is highly attenuated and masked by the molecular noise. Consequently, only the first receiver will be able to clearly identify "low" and "high" levels of concentration and thus to decode the message.

In conclusion, the attenuation introduced per unit of distance makes the communication only feasible in the short range, because a large number of molecules are required in order to successfully cover higher distances. These effects may even render unfeasible the approach of nodes competing for the channel, traditionally used in current wireless networks. Instead, cooperative schemes where nodes coordinate and jointly transmit the same 


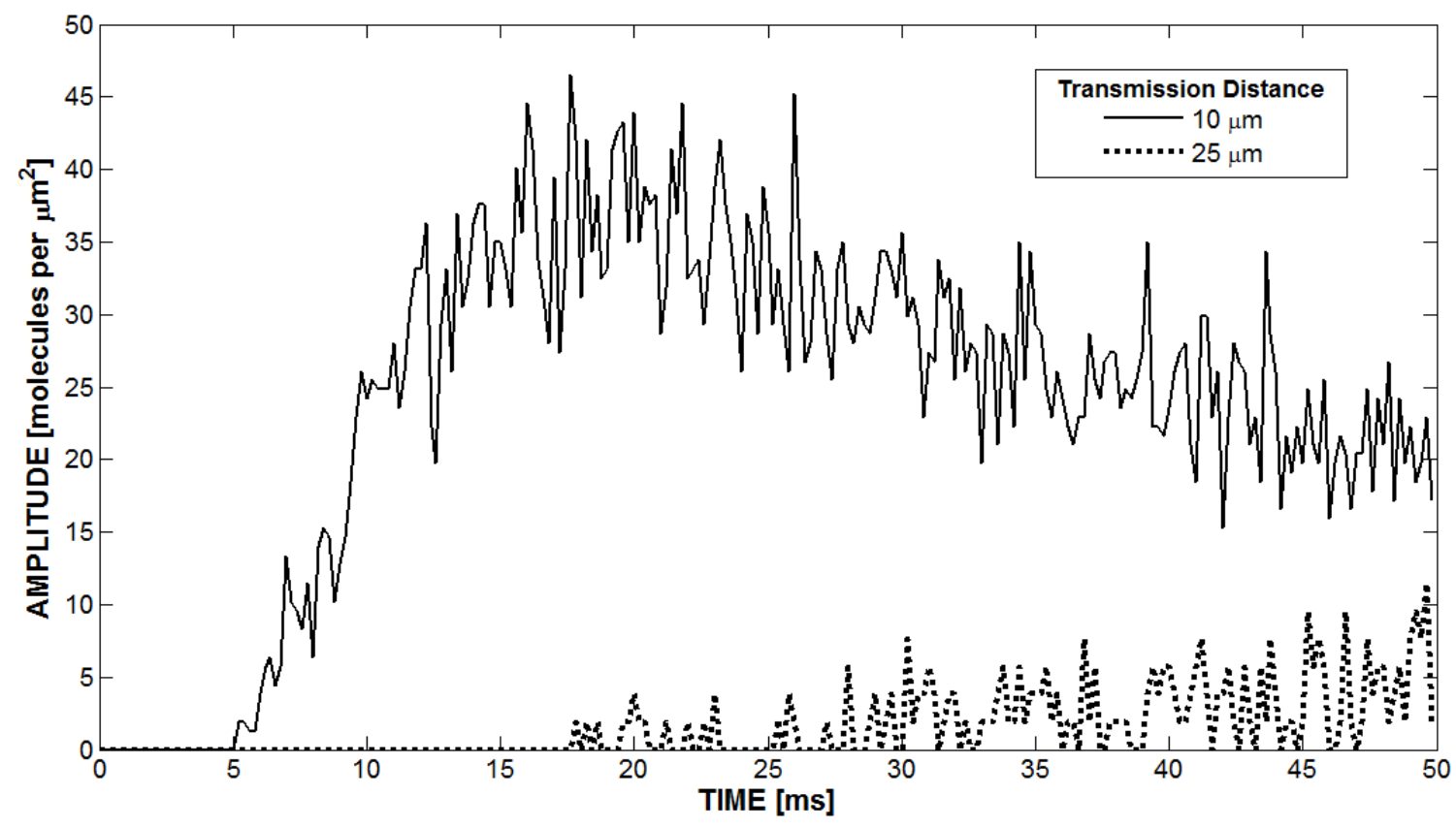

Figure 2.1: Reception of a pulse at distances of $10 \mu \mathrm{m}$ (solid line) and $25 \mu \mathrm{m}$ of the transmitter (dotted line).

signal, amplifying it, may allow the implementation of diffusion-based molecular communication. The main challenge then is how to coordinate the action of a group of nanomachines to accomplish the cooperation desired. We propose Quorum Sensing [26] as a way to coordinate the emission of a group of transmitters so that higher distances can be covered while relaxing the power consumption constraints.

The remainder of this thesis is organized as follows. Chapter 3 will be devoted to explaining in detail the biological phenomenon of Quorum Sensing and how will it help to address the attenuation problems of molecular communication. Next, the simulator and the different assumptions considered throughout the work are pointed out in Chapter 4.1.1. Using this framework, an analytical model of Quorum Sensing is developed and validated in Chapter 5. Finally, the signal amplification scheme and some associated results are introduced in Chapter 6, while the conclusions are presented in Section 7.2. 


\section{Chapter 3}

\section{Quorum Sensing}

Quorum Sensing is a biological process by which bacteria are able to communicate via signaling molecules called autoinducers. Major discoveries regarding the phenomenon of bacterial communication were made in the $60 \mathrm{~s}$ and the $70 \mathrm{~s}$, yet it was not until the $90 \mathrm{~s}$ that Quorum Sensing was concisely defined. W. Claiborne Fuqua, one of the most cited scientists that pointed out the existence of this process, stated that "groups of bacteria exhibit cooperative behavioral patterns" [26]. Precisely, by means of Quorum Sensing, bacteria are somewhat aware of the cell population density, and use that information to regulate their gene expression in a collective manner. Considering that the gene expression determines the behavior and functions of a living organism, Fuqua's initial observation was on the right track.

The evolutionary reason behind the communication capabilities of bacteria is quite clear. Quorum Sensing enables the control of bacterial functions or processes that are unproductive when undertaken by an individual bacterium but become effective when undertaken by the group [36]. For instance, many bacteria species need to launch attacks in order to survive or spread. If a bacterium alone launches an attack, host's defenses will eliminate the threat immediately. Whereas if a large group of bacteria launches an attack, the success rate rises enormously.

Fuqua was the first one to introduce the term "Quorum Sensing", which summarizes the concept, as the definition of Quorum is, "the number of members of a group or organization required to be present to transact business legally" [18]. Then, Quorum Sensing would be, 
in plain words, to make sure that there are enough members of the group to carry out a certain function.

The importance of Quorum Sensing is indisputable. Actually, it has been described as "the most consequential molecular microbiology story of the last decade" [40]. In fact, Wagner et al. [78] report that in P. aeruginosa up to 394 genes are activated by Quorum Sensing while 222 are repressed. In a recent review [42], it is estimated that between $6 \%$ to $10 \%$ of the whole genome is affected by this mechanism. These numbers can give us an idea of how relevant is Quorum Sensing in the cycle of life of bacteria, and the objective is to use this effective process to enable collectiveness between our nanomachines.

Next sections will be devoted to giving more insight about this bacterial phenomenon. In Sections from 3.1 to 3.3 we will thoroughly explain several aspects found in the literature, such as the principles, types and combinations of Quorum Sensing systems; whereas Sections 3.5 and 3.6 we analyze Quorum Sensing from a communication perspective and propose some applications.

\subsection{Principles and Mechanisms}

As Bonnie Bassler summarized in [9], Quorum Sensing is achieved through the production, release, and subsequent detection of and response to threshold concentrations of autoinducers.

Bacteria produce and emit sychronization molecules which diffuse in the medium. These molecules, called autoinducers, have the ability of triggering the release of more of the same kind, when sensed. Hence, as the population of bacteria grows, the extracellular concentration of autoinducers increases as well. If, at a certain point, the concentration of synchronization molecules reaches a critical threshold, it means that a certain population has been attained. That situation is sensed by the group, which responds to it with a population-wide regulation of the gene expression.

The fundamental part of the process can be regarded as a communication mechanism. The bacterium encodes the message through the synthesis of the autoinducers, and the proceeds to transmit it by means of secretion. The autoinducers propagate following the

Cooperative Signal Amplification for Molecular Communication in Nanonetworks 
diffusion laws until arriving to a destination, which will receive it through ligand-binding mechanisms. The global message is encoded in the density of particles, which is later decoded and understood as a gene regulation expression.

\subsubsection{Autoinducers}

Quorum Sensing is a really common process between bacteria. Many different species use it for their purposes in a wide range of possibilities. The type of autoinducer involved in the communication determines if two distinct species of bacteria are in the same conversation or not.

As stated before, an autoinducer is a tiny molecule which triggers the emission of more particles of its kind. Which species are going to be able to bind and sense them is determined in its chemical composition. Thus, there will be autoinducers that will enable intraspecies cell to cell communication, and others regarded as interspecies cell to cell communication. Interspecies communication allows the coexistence of different species in highly ordered communities, in which each of them carries out a specific subset of functions [9]. Some autoinducers, such as AI-2 and its synthase LuxS, even being extremely small (up to $4.5 \AA$ ), are to be considered as a kind of universal signal. This is due to the fact that this type of autoinducer has been identified in many species, which even differ in the type of apparatus used in its detection (see Section 3.2). The use of LuxS has been identified in more than 20 different species; a list can be found in [8].

\subsubsection{Thresholds}

The behavior of Quorum Sensing bacteria is determined by the concentration of autoinducers that they sense in the environment. Changes in that behavior are consequence of variations in the concentration, and several thresholds determine when these changes occur.

- Activation Threshold: as it has been explained in Section 3.1, when the concentration of autoinducers reaches a certain threshold, the colony performs a populationwide regulation of the gene expression. Therefore, all the bacteria of the colony change their behavior at once. From now on, we will refer to this critical value as "activation 


\section{A Acyl-Homoserine Lactone Autoinducers}
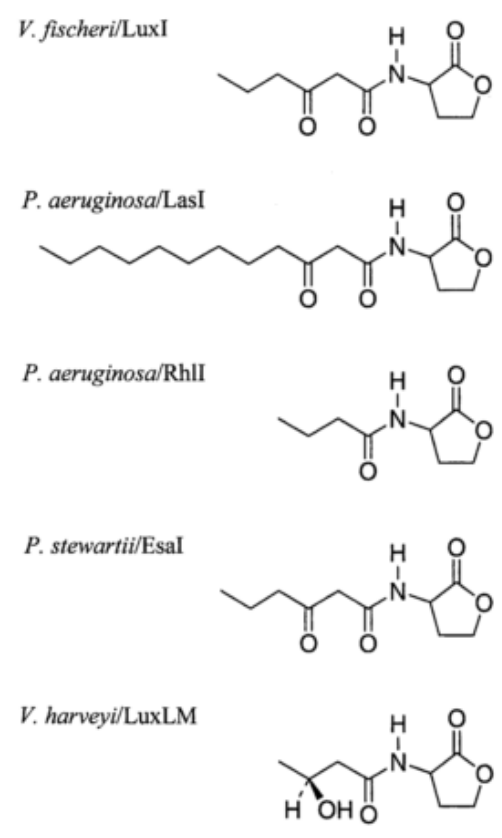

\section{AI-2 \\ V. harveyi/LuxS}

\section{B Oligopeptide Autoinducers}
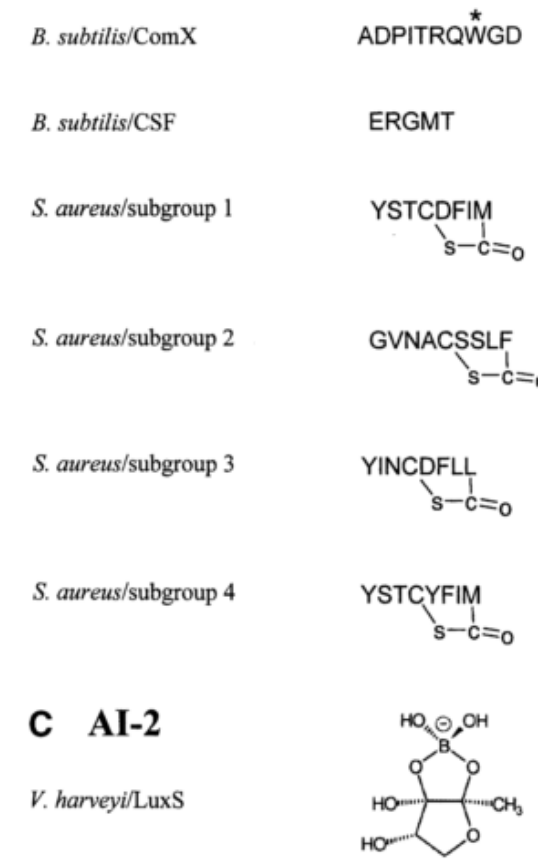

Figure 3.1: List of autoinducers, type, chemical structure, and species that use them.

threshold". However, when the behavior of Quorum Sensing is studied in depth, other threshold values appear.

- Autocatalytic Threshold: it has been also explained that bacteria emit autoinducers, particles that trigger the release of more of the same kind. By default, the autoinducers are synthesized at a basal or nominal rate. With increasing cell density, the extracellular concentration of autoinducers also increases. When this concentration reaches a certain threshold, referred as "autocatalytic threshold", the rate of emission of autoinducers rises dramatically. This is due to the fact that after surpassing this threshold, the autoinducers are synthesized by means of autocatalysis.

Autocatalysis is a chemical reaction widely known and studied. In this case, the reaction product is itself the catalyst for that reaction, thus creating a positive feedback loop. This serves as an explanation of how an autoinducer triggers the synthesis and 
emission of more particles of the same kind, and how the rate of emission in this situation is much higher than the nominal rate.

\subsection{Types of Quorum Sensing Systems}

Systems based on the principles of Quorum Sensing can be classified into three primary classes depending on the type of autoinducers involved and the internal reaction that is triggered when quorum is reached. Examples of these systems are shown in Figure 3.2.

- Gram-negative bacteria, such as Pseudomonas aeruginosa, Agrobacterium tumifaciens or Vibrio Fischeri rely on LuxI and LuxR enzymes. LuxI is the one in charge of the synthesis and emission of autoinducers of the family called AHL (standing for acylated homoserine lactone, see examples in Figure 3.1a), and LuxR binds to the received AHL to control the target genes. This is the most usual type of system, as it has been observed in more than 70 species.

- Gram-positive bacteria, such as Bacillus subtilis or Staphylococcus aureus use oligopeptides as autoinducers. These particles are produced inside the cell and modified before secretion, to distinguish them from oligopeptides secreted by other species of bacteria (see Figure 3.1b). At reception, proteins called sensor histidine kinases (SHK) detect the extracellular concentration of oligopeptides. When the activation threshold is surpassed, these proteins activate the response regulators (RR) by means of phosphorylation $(\mathrm{P})$, which consumes energy $(\mathrm{ATP} \rightarrow \mathrm{ADP})$. These response regulators are the ones that control the change in the gene expression.

- Hybrid. In this class, bacteria count on a system which is a hybrid between the two previously stated canonical options. Vibrio Harveyi or Salmonella typhimurium are considered to be of this type of bacteria, as they combine AHL-based systems with oligopeptide ones. For instance, the hybrid system in Figure 3.2 produces two different autoinducers (AI-1 and AI-2). AI-1 is from the AHL family of autoinducers, like the ones used in Gram-negative bacteria, whereas AI-2 is produced by the enzyme LuxS and has no resemblance with other autoinducers (see Figure 3.1c). Both, at

Cooperative Signal Amplification for Molecular Communication in Nanonetworks 
detection, trigger the regulation of the gene expression through phosphorylation, like Gram-positive systems do. In this case, a protein called histidine phosphotransfer (HPt) is able to react to the sensing of both autoinducers, transferring its activation to the response regulator.

Further biological details on the enzymes and reactions that are involved in each type of Quorum Sensing can be found in [8].

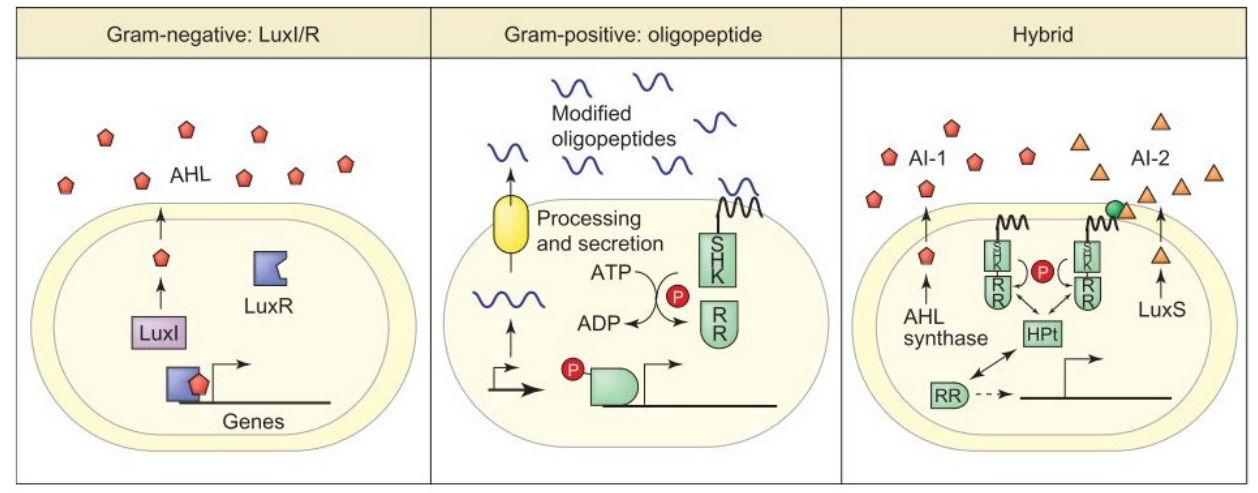

Figure 3.2: Types of Quorum Sensing systems, from [36].

\subsection{Combination of Quorum Sensing Systems}

So far, we have seen that bacteria have a way to communicate with members of its own species, and even with members of other species. The complexity of the Quorum Sensing apparatus shown seems to be enough for living organisms not larger than a few micrometers. However, there have been cases reported about bacteria containing several, oftentimes overlapping, Quorum Sensing systems. Two clear examples are Rizhobium leguminosarum, which contains at least four distinct Quorum Sensing processes [42]; and Pseudomonas aeruginosa, in which it has been observed that it is composed of two overlapping systems. Other details and explanations about P. aeruginosa are shown in [40]. Some studies go further, and assert that those systems are organized in series and/or parallel.

In the case of $P$. aeruginosa, the systems act in series to regulate two overlapping subsets of genes, which assures a sequential activation of the two groups [71]. Further- 
more, supposing that a non-activation of one of the systems does not affect any genes, the overlapping set of genes will respond to the equivalent of an OR gate between the two systems.

On the other hand, there exist at least two documented cases in which Quorum Sensing works in parallel: Vibrio harveyi, shown in Figure 3.3, and Bacillus subtilis.

- Vibrio harveyi: in this case, the systems converge to regulate a common set of target genes [50]. Enzymes LuxM and LuxS are responsible for the production of two types of autoinducers, namely, AI-1 and AI-2. Autoinducers of the first kind are sensed by the enzyme LuxN, whereas AI-2 molecules are captured by the periplasmic binding protein LuxP, and later sensed in the cytoplasm by the enzyme LuxQ. The information provided by those two autoinducers is integrated by means of joint phosphorylation, triggering a cascade of reactions in different enzymes (LuxU, LuxO, and later LuxR) only if there is enough concentration of both autoinducers. Eventually, the enzyme LuxR will regulate the gene expression with changes indicated in small regulatory RNAs (sRNAs + Hfq) present in the cell.

In conclusion, there are four different inputs for the combined system found in Vibrio harveyi: no autoinducer, AI-1 activation, AI-2 activation, or activation of both. The change of behavior (or output) will only occur when both autoinducers AI-1 and AI-2 are present. Just like an AND gate.

- Bacillus subtilis: these bacteria use parallel systems to respond to different autoinducers, which control different groups of genes. Concretely, one of them controls the behavior in terms of competence, and the other in terms of sporulation. The presence of an autoinducer called ComX will trigger competence behavior, only if another autoinducer called CSF, is not sensed. This is due to the fact that both autoinducers have inverse chemical consequences inside the cell (namely, phosphorylation and dephosphorylation, being phosphorylation responsible for the activation of the gene regulation) [41].

Cooperative Signal Amplification for Molecular Communication in Nanonetworks 


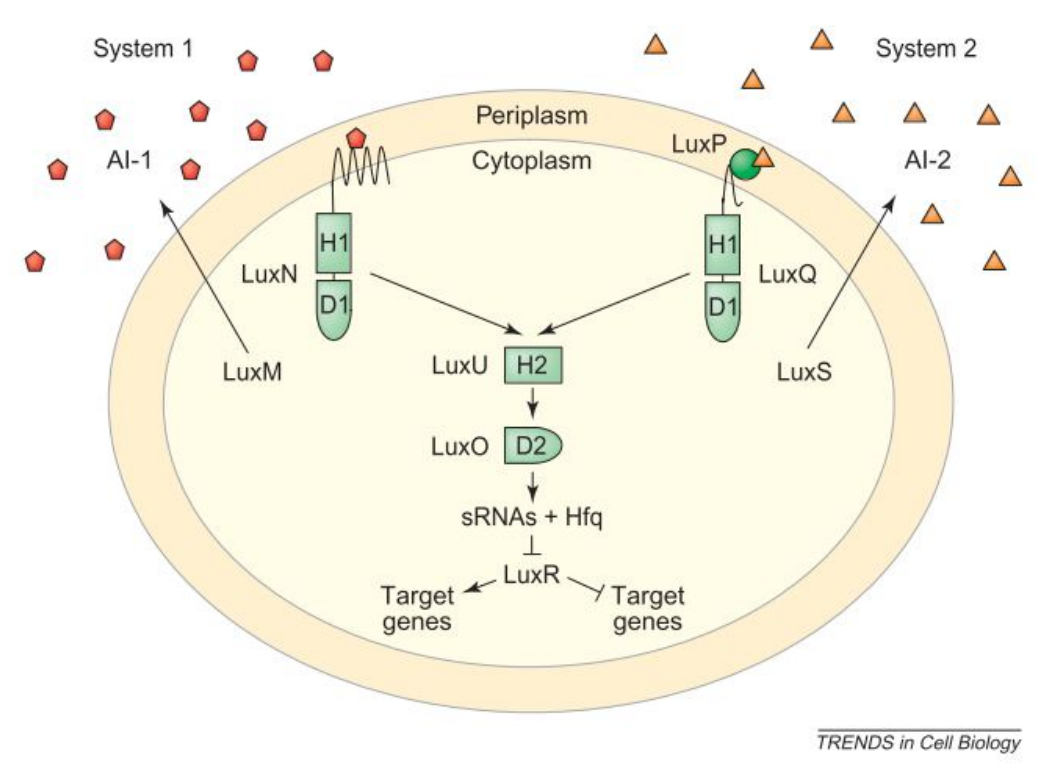

Figure 3.3: Example of parallel combination. V. harveyi case, from [36].

Then, it is clear that if the presence of autoinducers ComX and CSF are represented by boolean parameters $\mathrm{A}$ and $\mathrm{B}$, respectively, the output (activation of competence) is determined by the expression $C=A \bar{B}$.

It is worth noting that all the combinations of canonical systems have been observed in nature. For example, $P$. aeruginosa uses two gram-negative schemes, B. subtilis consists of two gram-positive systems, and $V$. harveyi is a hybrid scheme. In fact, the canonical hybrid system can be regarded as a simple combination of two Quorum Sensing systems.

As a conclusion, the observations made in this section should catch the attention of the scientific community in the sense that the embedding of complex systems based on Quorum Sensing in nanomachines can be possible.

\subsection{Bacterial Behaviors Controlled by Quorum Sensing}

As it has been stated throughout this section, Quorum Sensing is a process found in nature that allows bacterial communication. What is more, Quorum Sensing allows bacteria to have social interactions in order to synchronize their actions. Hence, behaviors controlled 
by Quorum Sensing are those that take advantage of this synchronization feature, enabling bacteria to act as multicellular organisms.

Also it has been affirmed that the change of behavior observed after Quorum Sensing depends solely on the regulation of the gene expression. Numerous examples of different behaviors can be found in the literature (see Table 3.1). Bioluminescence, virulence, biofilm formation, sporulation, and mating are processes that can be controlled by Quorum Sensing, since they become a lot more effective when undertaken by a group.

As a consequence of the study of all these mechanisms that use the principle of Quorum Sensing, a lot of ideas for novel applications in the nanoscale have arisen, and some of them will be presented in Chapter 3.6. Whereas in this section, a brief explanation of the principal processes that involve Quorum Sensing and that can be seen in nature, is developed. After that, an antagonist mechanism called "Quorum Quenching" is described, owing to its potential as an application.

\begin{tabular}{|l|l|}
\hline Species & Functions regulated by Quorum Sensing \\
\hline Actinobacillus actinomycetemcomitans & Virulence factors \\
Campylobacter jejuni & Motility \\
Escherichia coli W3110 & Cell division, DNA processing, cell shape \\
Neisseria meningitides & Bacteremic infection \\
Photorhabdus luminescens & Carbapenem (antibiotic) biosynthesis \\
Salmonella typhi & Biofilms \\
Vibrio harveyi & Bioluminescence \\
\hline
\end{tabular}

Table 3.1: Examples of functions controlled by Quorum Sensing (from [79]).

\section{Bioluminescence}

This characteristic is what lead to scientists to think that there is a mechanism through which bacteria were aware of their own population density. Seen in marine bacteria species Vibrio fischeri and its free-living relative $V$. harveyi, the autoinduction of bioluminescence 
was described in the early 1970s. In this case, it is clear that the intensity of the light of one bacterium is too low to be seen or used in a beneficial way for the species, making Quorum Sensing a necessity.

The example usually described to illustrate this case refers to the Euprymna scolopes. It is a small squid, $40 \mathrm{~mm}$ long in average, that is indigenous to the Hawaiian archipelago. It lives in shallow waters associated to coral reefs and sand flats, where it buries itself during the day to escape predators. At night, the squid comes out to hunt, therefore being more vulnerable at this time. Actually, the moonlight goes through the shallow waters and is able to cast shadows on the sand surface, situation that can be used by predators to detect the position of the squid and catch it.

Here is when the symbiosis between E. scolopes and the bacteria $V$. fischeri comes into play. The squid hosts a small colony of those bacteria in an organ that lies in the mantle cavity of the animal (given in Figure 3.4). This colony remains deactivated during the day, but growing inside the organ. At night, the colony will have reached a certain population and Quorum Sensing will work, thus activating the bioluminescence. Light emitted by the bacterial symbionts is used by the squid to camouflage by means of counterillumination. This is, the animal controls the intensity of the projected light, matching the moonlight shining down and eliminating the shadows. By the time the squid has to bury again in the sand, it frees a $95 \%$ of the colony because the host is not able to sustain such a big number of bacteria. Therefore, the cycle repeats as during the day the remaining bacteria will reproduce again so at night the population is high enough to activate.

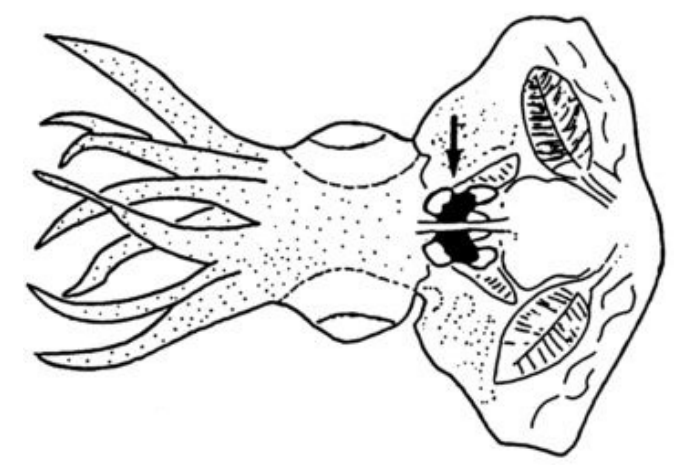

Figure 3.4: Diagram of the ventral view of an E. scolopes sample. 
A more thorough depiction of the symbiosis between those two species can be found in [65], and a detailed analysis of the gram-negative process that occurs in the activation of $\mathrm{V}$. fischeri in the same case, is offered in [26].

\section{Virulence}

To survive, many species of bacteria have to launch an attack to their host to cause a certain reaction. For instance, when a certain environment starts to lack nutrients necessary for the bacteria to persist, they might attack to force host-to-host transportation. So, basically, the attack will make the host cough, sneeze, bleed or to have whichever reaction serves to get the bacteria out and in a position to infect new hosts, always in search for a favorable environment.

However, as it has been explained in the introduction of this chapter, host defenses can get rid of the bacteria if they launch an attack too early. Therefore, Quorum Sensing is necessary for the colony to delay virulence factor production, until cell number is high enough that secretion of virulence factors will result in a productive infection.

\section{Biofilm Formation}

Both bioluminescence and virulence seem to be, in principle, processes that would only need the intraspecies cell-to-cell communication, if we suppose that virulent species do not use interspecies awareness of population density for its purposes. However, there are natural processes that take advantage of interspecies communication to coordinate the action with other kind of bacteria in a reciprocally beneficial way.

The formation of biofilms is a process in which interspecies communication has been observed. In biofilms, bacteria anchor themselves to a surface, facilitating the adhesion of more of them, forming a layer over the surface. In some cases, species of bacteria without attachment capabilities adhere directly to the colonists that previously anchored on the surface. It is during this process than interspecies Quorum Sensing takes place.

In fact, in biofilms bacteria are organized into elaborate framework. Different species are located in specialized regions, and a flow of nutrients is provided by aqueous channels through the entire structure. Moreover, biofilms are highly resistant to antibiotics and 
desiccation. Consequently, it is not strange that bacteria benefit from communal living, as it increases highly its chances of survival. Therefore, Quorum Sensing is essential as a way to communicate the different species.

For instance, AI-2, explained in Section 3.1.1 as a sort of universal autoinducer, has been shown to be required for mixed species biofilm formation between Streptococcus gordonii and Porphyromonas gingivalis, members of biofilm communities in dental plaque [36].

\subsubsection{Quorum Quenching}

It is not surprising that different species have developed strategies to interfere with Quorum Sensing, since Quorum Sensing controls fundamental processes involved with both bacterial physiology and virulence. Autoinducer antagonists, autoinducer destroying enzymes, and other mechanisms for consuming autoinducers are now known to enable "Quorum Quenching" [21]. Therefore, basically, Quorum Quenching can be regarded as all the mechanisms that prevent the correct operation of Quorum Sensing.

On one hand, autoinducer destroying enzymes or other consuming mechanisms would reduce the concentration of autoinducers to a level below the threshold, thus avoiding the collective gene regulation. On the other hand, a flood of fake autoinducers or any particle that can act as an autoinducer for a specific species, would trigger the gene regulation in an unfavorable situation. Nevertheless, Quorum Quenching studies have been started recently, and they suggest that many other different mechanisms exist and await identification.

Examples of these mechanisms have been observed in both intraspecies (Staphylococcus aureus) and interspecies (some Bacillus) Quorum Sensing processes. The motivation for its use does not have to be necessarily competence, though. Bacteria can organize and sequence their actuation by the use of this process, and also Quorum Sensing. For instance, if a behavior (A) is not compatible with another one (B) in a community, the release of autoinducers to trigger the behavior A will come together with the release of Quorum Quenching agents for the behavior B.

The medical community has put a lot of effort in this point, due to the fact that it might be interesting for a really concerning area: antibiotics. In light of the resistance to antibiotics gained bacteria, a new way of defeating bacterial illnesses would be to alter

Cooperative Signal Amplification for Molecular Communication in Nanonetworks 
the normal course of Quorum Sensing, either with autoinducer destroyers, or causing a premature regulation of the gene expression.

\subsection{Communication Aspects of Quorum Sensing}

The Quorum Sensing phenomenon can be regarded as a communication mechanism. Each bacterium encodes the message through the synthesis of autoinducers and transmits it by means of secretion. The autoinducers propagate following spontaneous diffusion until they arrive to a destination, which will receive it through ligand-binding mechanisms.

One of the differences of molecular communication with respect to the traditional communication paradigms is the concept of global message. Molecular messages can be achieved by the accumulation of the contributions of several transmitters, in a process of inherent data aggregation. In the particular case of Quorum Sensing, the global message is encoded in the concentration of particles, which is later decoded and understood as a node density. From the communication perspective, we could define Quorum Sensing as a collective or cooperative communication scheme.

Also, special cases of noise, signal attenuation, and interferences have to be identified when considering Quorum Sensing as a particular case of molecular communication. For example, autoinducer-destroying enzymes can be considered a source of noise, since they diminish the concentration of autoinducers that a node will sense, therefore affecting the global message.

\section{Addressing}

Addressing in Quorum Sensing-enabled networks is directly connected with the autoinducers. The chemical structure of these particles determines which receivers will be able to sense them. On one hand, a huge variety of autoinducers enable intraspecies cell to cell communication, meaning that only nodes of the same species of the transmitter will be capable of receiving the message. From the addressing perspective, the address encoded in this type of autoinducers is inherently multicast. On the other hand, some particles are considered as some kind of universal signals (i.e. LuxS [9]), enabling interspecies cell to 
cell communication. The address encoded in this type of autoinducers can be considered as broadcast, as the message can be received by any node in the environment.

\subsection{Applications of Quorum Sensing}

Quorum Sensing and its antagonist process Quorum Quenching has attracted the attention of the scientific community. Knowing more about Quorum Sensing is knowing more about bacterial intraspecies and interspecies communication schemes and, in turn, more about how different species organize inside the same ecosystem. Also, the insight gained about Quorum Quenching is expected to allow scientists to modify the course of this natural synchronization process.

As explained in Section 3.4.1, the capability of controlling Quorum Sensing process would have a huge impact in the medical field, since it could be the key of new generation antibiotics. The trend about creating new antibiotics stems from the concern that arose around the fact that current antibiotics try to kill bacteria, resulting in the apparition of mutant varieties resistant to those antibiotics. A way to combat this situation is to deploy a regulated killing system [80] that controls and stabilizes actively the population of bacteria, preventing their activation. Alternatively, Quorum Quenching would jam the communication between members of the colony, in order to prevent the bacteria to attack, or to attack when they are not enough in number to be effective. In summary, affecting intraspecies systems, disease-specific antibiotics can be created; whereas affecting interspecies Quorum Sensing systems, broad spectrum antibiotics will be conceived [73].

Disrupting bacterial communications could be also the key to prevent the formation of harmful biofilms. For instance, bacterial biofilms formed in plastic catheters are the cause of severe infections [53]. Other examples can be found in Table 3.2 and include preventing the formation of biofilms in dental care or industrial environments: places in which certain biofilms are not welcome.

However, the objective of this work is to extract the communicative principles of Quorum Sensing and to analyze its possible applications in the field of nanonetworking. Indeed,

Cooperative Signal Amplification for Molecular Communication in Nanonetworks 


\begin{tabular}{|c|c|c|}
\hline $\begin{array}{l}\text { Representative } \\
\text { Entities }\end{array}$ & Effect on Quorum Sensing & Evidence of Benefit \\
\hline Azithromycin & $\begin{array}{l}\text { Reduction in LasI, Rh1I, autoinducers, } \\
\text { and virulence in } P \text {. aeruginosa }\end{array}$ & $\begin{array}{l}\text { Increased lung function in patients with } \\
\text { panbronchiolitis and } \mathrm{CF}\end{array}$ \\
\hline RIP, RAP vaccine & $\begin{array}{l}\text { Inhibition of the action of RNAIII- } \\
\text { activation protein in staphylococci }\end{array}$ & $\begin{array}{l}\text { Reduced virulence in various } S \text {. aureus an- } \\
\text { imal models; decreased biofilm production } \\
\text { on catheters by } S \text {. aureus and } S \text {. epidermis }\end{array}$ \\
\hline C30, C56 & $\begin{array}{l}\text { Inhibition of LuxR-dependent gene ex- } \\
\text { pression in Gram-negatives }\end{array}$ & $\begin{array}{l}\text { Increased clearance of } P \text {. aeruginosa in a } \\
\text { mouse model of pneumonia; reduced ad- } \\
\text { herence to catheters }\end{array}$ \\
\hline $\begin{array}{l}\text { Several compounds } \\
\text { isolated from plants }\end{array}$ & Degradation of HSL signal molecules & Various agricultural/horticultural benefits \\
\hline $\begin{array}{l}\mathrm{N} \text {-(2-oxocyclohexyl)- } \\
\text { 3-oxododecanamide }\end{array}$ & Antagonism of HSL activity & $\begin{array}{l}\text { Decreased biofilm production by } P \text {. aerug- } \\
\text { inosa in vitro }\end{array}$ \\
\hline $\begin{array}{l}3 \text {-oxo- } \mathrm{C}_{12} \text {-HSL-prot. } \\
\text { conjugate vaccine }\end{array}$ & $\begin{array}{l}\text { Reduced pulmonary TNF-alpha aggre- } \\
\text { gation in pneumonia }\end{array}$ & $\begin{array}{l}\text { Protection from lethal } P \text {. aeruginosa lung } \\
\text { infection in mice }\end{array}$ \\
\hline
\end{tabular}

Table 3.2: Potential therapeutic options associated to Quorum Quenching (from [48]).

as Quorum Sensing is a process achived by bacteria by means of molecular communication, we believe that molecular nanocommunication networks best fit the characteristics of Quorum Sensing. Nevertheless, molecular communication presents important challenges. In this section, we will present different schemes based on Quorum Sensing that could help to solve these challenges: global synchronization, reliability, detection of complex events, distributed logic gates and signal amplification will be briefly introduced. The signal amplification scheme represents the major contribution of this work and will be described and modeled in more detail in Chapter 6 .

\section{Global Synchronization}

The first and most clear application is synchronization between nodes. As it was presented in [2], Quorum Sensing can be thought as a synchronization tool for dense networks, in which bacteria are the nodes that communicate through the emission and sensing of autoinducers. 
These nodes start emitting autoinducers with no apparent synchronization between them, but at the end of the process, they all activate at the same time.

The same principle can be applied in networks in general and nanonetworks in particular. A nanomachine starts emitting autoinducers upon receiving a certain quantity of molecules of the same type. If the node density is high enough, a given amount of autoinducers concentration will be present in the environment. At that point, all the nodes activate in a synchronous manner.

This possibility was originally inspected in previous work [1]. Two different designs were presented so that nanomachines could perform either "collective activity synchronization" or "collective actuation after localized sensing":

- Nanomachines programmed for collective activity synchronization simply make use of Quorum Sensing as a feature to coordinate the action of all the units present in the medium. A fixed number of these nanomachines will be deployed in a target environment, and this group will not grow since these nanodevices do not have self-reproductive capabilities. In general terms, the behavior of these nanomachines consists, by default, of the release of autoinducers depending on the concentration sensed. When this concentration surpasses a certain threshold, the processing unit alerts the control unit, which will make the actuators perform the programmed "activity". This way, Quorum Sensing could be used for the synchronization of bacterial clocks, otherly referred to as cellular oscillators, to generate a global clock [16, 27].

- The objective of nanomachines performing collective actuation after localized sensing is to achieve a global reaction as a response to a certain localized stimulus. Figure 3.5 exemplifies the process: (a) nanomachines start a Quorum Sensing process when they sense a certain stimulus, (b) alerting neighbouring cells and (c) eventually activating the whole colony of nanomachines in order to achieve collective actuation. For instance, killer automata could be implemented in order to effectively eliminate cancer cells, which release specific immunodepressor chemicals [48]. This way, elimination of tumors in a localized, minimally invasive, and extremely low scale can be achieved. 


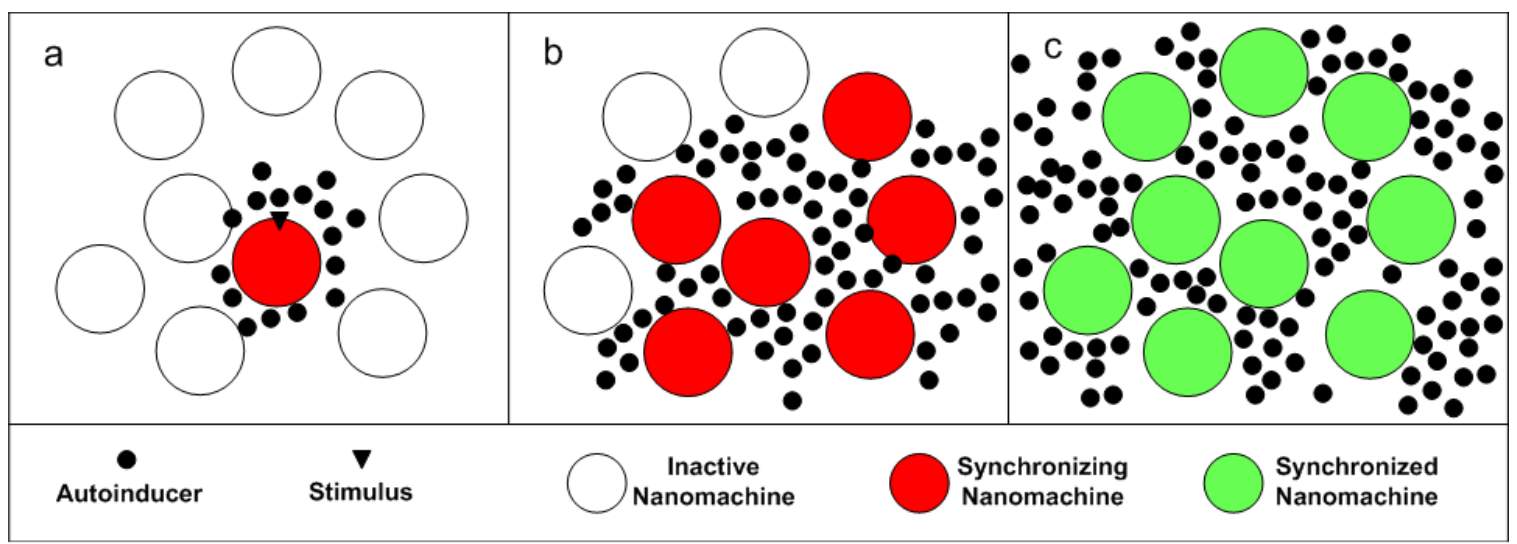

Figure 3.5: Example of a collective actuation after localized sensing process.

\section{Reliability}

Novel nanosensors, which are envisaged to be integrated in nanomachines and connected forming Wireless NanoSensor Networks (WNSN), are able to detect chemical compounds in extremely low concentrations [68]. The implications of this feature are twofold: on the one hand, the sensitivity of these sensors is unprecedented, enabling the development of novel applications. On the other hand, the reliability of the system might be affected, in the form of false positives.

We propose the use of Quorum Sensing in order to tackle this problem. In wireless sensor networks in general and WNSN in particular, when an event is detected it is sent to a special node called sink. Our objective is to make several nodes reach quorum before sending the result to the sink. This way, several nodes "vote" if they have detected the event or not. If enough votes are counted, the group will send the result to the node in charge to process this information. If this sink is far away, the sensor nodes could also benefit of this quorum process in order to perform a cooperative amplification of the pulse to be sent, as proposed in Section 6 .

The scenario is the following: we have deployed a dense nanosensor network to detect an extremely small concentration of cancer cells. A node will only start emitting autoinducers if and only if it senses a certain event (differently from other cases where nodes emitted autoinducers upon receiving autoinducers). This way, the concentration of autoinducers will 


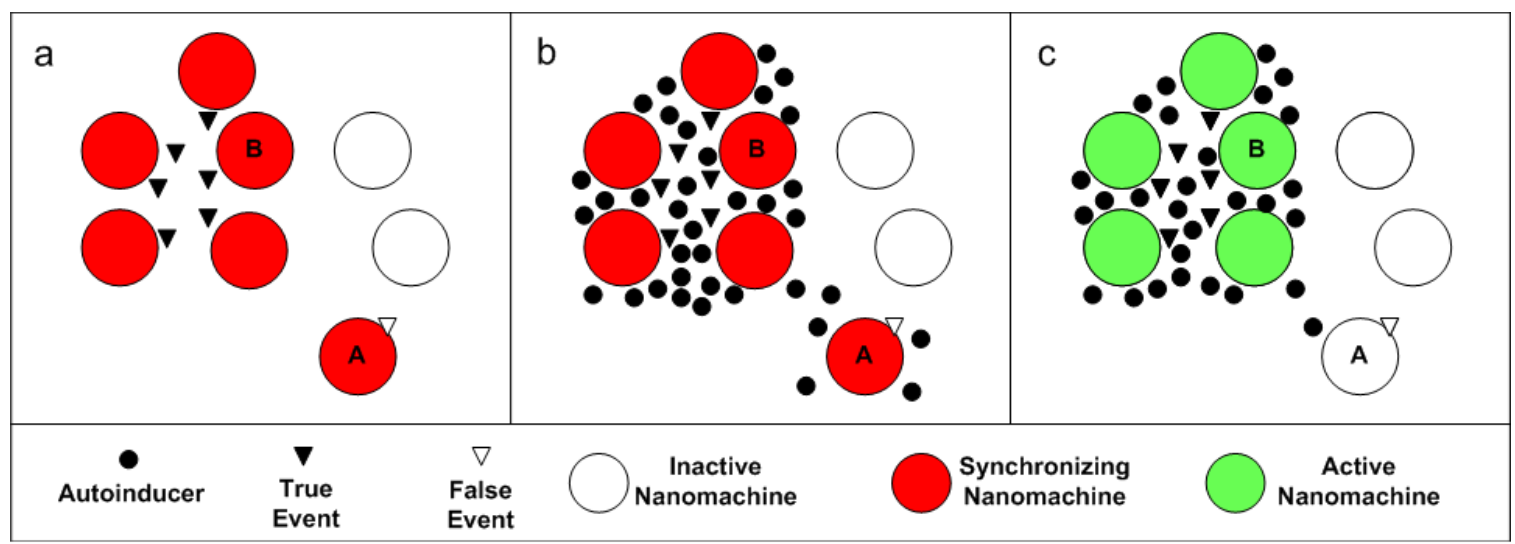

Figure 3.6: Example of reliable sensing accomplished by means of quorum.

be proportional to the number of nodes that detected the event, not to the node density. Thus, when a given number of nodes has performed the detection, the concentration of autoinducers will reach the activation threshold. Then, this group of nodes will activate and will perform the proper actuation, reducing notably the false positive probability.

An example of such scheme is shown in Figure 3.6. Node A detects a certain event that would cause a false positive. That event triggers the emission of autoinducers, but quorum is not achieved since neighbouring cells do not sense the same situation. This way, a false positive is avoided. On the other hand, node B and its neighbours detect a certain event and start emitting autoinducers. As several nodes sense the same situation, quorum is achieved and the group of nodes activates to, for instance, report the event to the sink of this WNSN.

Intuitively, the more nodes are involved in a sensing process, the lower will be the probability of assessing a false positive. Also, as the concentration of autoinducers increases proportionally to the number of nodes emitting, the activation threshold will determine the number of nodes needed to reach quorum. Therefore, setting a high threshold would increase reliability but would, at the same time, decrease locality and sensitivity in the detection. Moreover, as we will see in Chapter 5, the delay of the Quorum Sensing process grows proportionally to the number of nodes involved. Hence, a compromise between reliability and delay should be reached. Setting a high threshold would increase the index of reliablity 


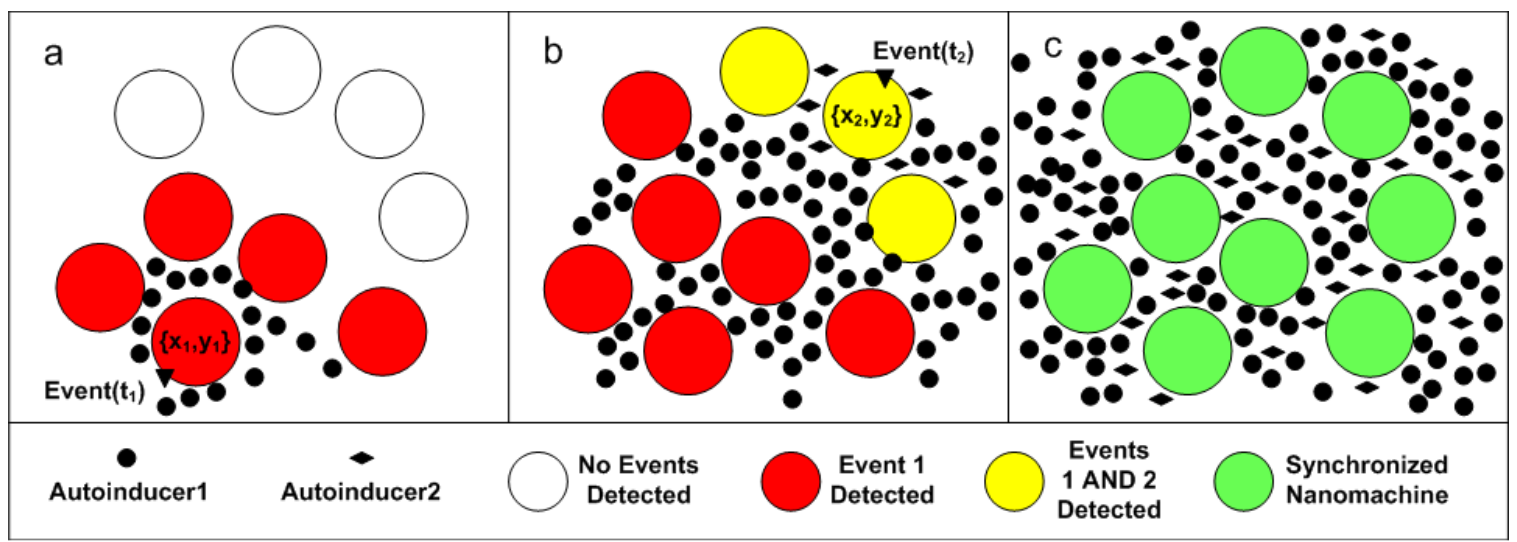

Figure 3.7: Example of the detection of a complex event.

but with a significant delay, whereas a low threshold would imply a faster actuation but with a higher false positive index.

\section{Detection of Complex Events}

In classical Wireless Sensor Networks, simple events are detected. Often, sensors are programmed with protocols that aggregate data that go through them so that complex events can be detected via software. However, we believe that by using combinated Quorum Sensing systems, the detection of complex events can be achieved without relying on programmed algorithms, thus reducing the computational charge that nodes suffer.

The scenario is the following: the considered nodes will start emitting autoinducers if they detect a given event or if they detect autoinducers of the same type, and will only activate when they detect the presence of two different autoinducers. An example is shown in Figure 3.7: the node located at the point $\left\{x_{1}, y_{1}\right\}$, which is initially at rest, starts emitting autoinducers when it detects a given event in time instant $t_{1}$. When the surrounding cells sense an autoinducer, they also start emitting autoinducers. This way, after a certain time, a group of nodes will be aware of the event intially detected. When the node located at coordinates $\left\{x_{2}, y_{2}\right\}$ detects a second event, it starts emitting a second type of autonducers. Thus, the group of nodes is eventually affected by two different types of autoinducers, achieving synchronization by all the nodes in the cluster. 
As explained in Section 3.3, some bacteria react to different combinations of autoinducers. Thus, with this scheme, we could detect complex events; this is, events that are separated in space or time. For instance, in the scenario explained before, the simultaneous detection of the two different autoinducers would mean that two determined events happened in that area. For instance, this could serve to detect the coexistence of two chemicals that should not be in the same environment, because their combination is toxic, or because it demonstrates the existence of a tumor.

\section{Distributed Logic Gates}

Another conclusion that can be extracted from Section 3.3 is that the series or parallel combinations of Quorum Sensing schemes are perfect for the engineering or assembly of distributed and synchronized logic circuitry. For instance, if the levels of autoinducers A and B are seen as digital 'high' and 'low' levels, the parallel systems described above would both act as AND gates of the two levels (AB for the Vibrio harveyi family and $A \bar{B}$ for Bacillus subtilis), achieving a synchronized response of a group of entities.

However, inspecting this possibility and its potential applications is out of the scope of this thesis and remains as future work.

\section{Signal Amplification}

In this work, we propose to apply the principles of Quorum Sensing to achieve signal amplification in diffusion-based molecular nanonetworks. By making use of this cooperative approach, a group of nodes would be able to synchronously emit a certain message. The proposed scheme has two differentiated parts:

- When a node has information to transmit to other distant nodes, it releases synchronization molecules (autoinducers) to start a Quorum Sensing process with its surrounding neighbours. The neighbouring nodes also start the release of synchronization molecules, increasing the concentration of these particles. Eventually, the group of nodes will synchronize upon sensing a certain concentration of autoinducers. 


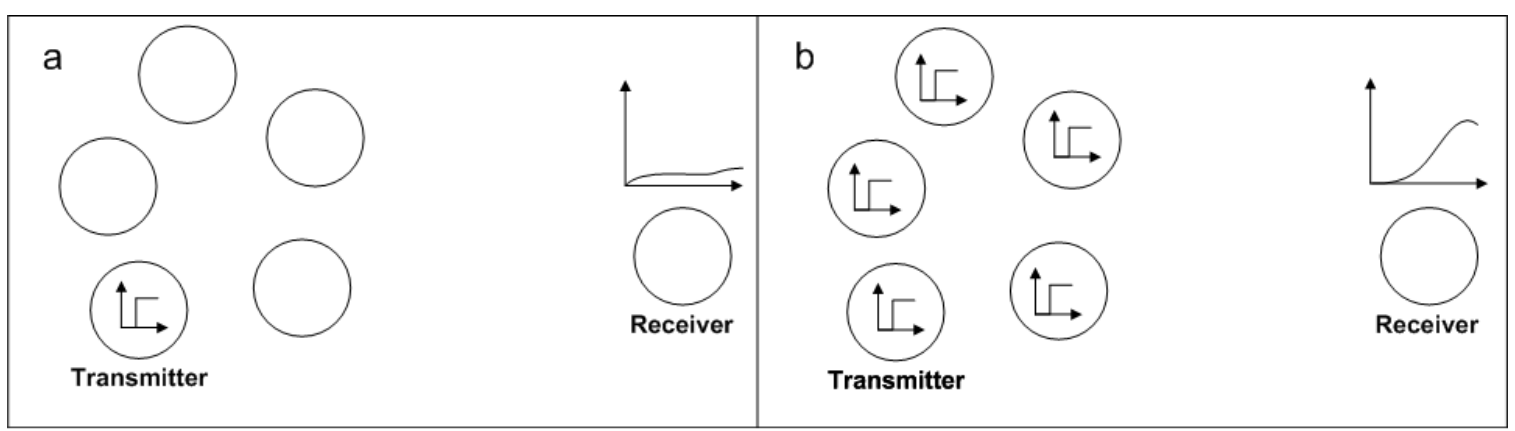

Figure 3.8: Example of a transmission (a) without and (b) with cooperative amplification.

- After this synchronization phase, the original transmitter and its neighbours will jointly and coordinately transmit a given message by using communication molecules. Since the channel is Linear and Time-Invariant (see Section 5.2 for more details) the contributions of the participating nodes are aggregated and the signal is effectively amplified. The specific information encoded into the synchronized signal is left out of the scope of this work, but it is worth to note that it can be either a pulse, or a (pre)configured sequence of pulses. Furthermore and since hundreds of different autoinducers exist (see Section 3.1.1), different autoinducers could trigger different synchronized signals.

Figure 3.8 shows the difference between the classical point-to-point communication and the proposed cooperative scheme. In the first case, the transmitter directly sends a pulse to the receiver; whereas in the second case, the transmitter synchronizes its emission with its neighbouring nodes. The received pulse will be the aggregation of the contributions from the nodes the transmitter has synchronized with.

The remainder of this work will be devoted to further explain this cooperative signal amplification scheme. In Chapter 5 we provide an analytical model of Quorum Sensing that proves the dependency between concentration of synchronization molecules and the node density. This model is developed and validated through simulation by using the framework that is introduced in Chapter 4.1. It will allow the evaluation of two important figures that will be later discussed: the activation threshold of the nodes, and the delay introduced by the synchronization phase. The activation threshold is directly related to the number of 
nodes that will be involved in the transmission, which is, in turn, proportional to the level of amplification needed. On the other hand, the delay of the synchronization process will determine the time between two consecutive symbols.

Taking under consideration the analytical model of Quorum Sensing, the signal amplification scheme is eventually extended and further analyzed in Chapter 6 . 


\section{Chapter 4}

\section{Framework}

The work presented in this thesis needs a certain level of abstraction. Some cellular processes and phenomena underlying in the nanoscale are relatively unknown and thus several assumptions are needed in order to model them. For instance, the diffusion of particles in a fluidic medium can be modeled by means of the Fick's laws of diffusion with several conditions (see Section 4.1.2).

In this section the reader will find different considerations needed to fully understand the analytical model of Quorum Sensing developed in Section 5, as well as some details regarding the simulator that has been used to validate the results contained in the aforementioned section.

\subsection{Analytical Environment}

Quorum Sensing relies on the production, emission and sensing of autoinducers. These autoinducers propagate through the environment in which the bacteria are living, and we need to know that environment in order to be able to model the phenomenon of Quorum Sensing. The variety of species of bacteria that perform Quorum Sensing is astonishingly wide, with more to discover. That means that the environments in which one can find these

species are also diverse. Nevertheless, some assumptions regarding those sceneries will be made in the next section, in order to simplify the model. 


\subsubsection{Assumptions}

We can say that Quorum Sensing will take place in a homogeneous and finite space, that can be also be considered as a Linear and Time Invariant molecular channel. The agents performing Quorum Sensing will be static, identical in nature, transparent with respect to other particles and will be arranged forming a perfect tridimensional grid. Finally, autoinducers are particles that will propagate following a process called molecular diffusion.

The set of assumptions will be depicted following a top-bottom approach. Aspects concerning the medium will be explained first, followed by assumptions related to the different nodes performing Quorum Sensing. Finally, the model of the physical process of molecular diffusion is explained in detail.

\section{Finite and Homogeneous Environment}

Reported species of bacteria range in size from barely $50 \mathrm{~nm}$ up to $750 \mu \mathrm{m}$ the biggest. However, spherical or not, the most usual sizes are of the order of a few micrometers. Also, colonies of these bacteria can reach populations of approximately $10^{5}$ individuals, or more, like in the case of Vibrio fischeri [64]. Then, cultures of bacteria participating in Quorum Sensing do not occupy spaces larger than a few millimeters. Not in vain, cultures not exceeding a diameter of $2 \mathrm{~mm}$ are considered small, being large if they surpass the $5 \mathrm{~mm}$ of diameter [35]. The purpose of this discussion is to make clear that spaces in which bacteria act in Quorum Sensing are small and will be considered as finite: events taking place at several centimeters of distance will not affect the course of action of the culture of bacteria, at least in the short term.

Also, real environments generally have a set of properties which by default vary spatially and temporally. This is because elements that configure those properties are changing in a constant fashion. However, considering a heterogeneous space, meaning that its properties vary as a function of space and time (randomly or not), would add an unacceptable degree of complexity to the model. In our case, the extension of bacterial colonies is commonly small, so that changes of the environment properties are limited over space. Hence, the space will be considered homogeneous, meaning that its properties will be the same throughout all its extension.

Cooperative Signal Amplification for Molecular Communication in Nanonetworks 


\section{Linear and Time Invariant Channel}

As seen in Section 3.5, Quorum Sensing can be seen as a molecular-driven communication process, in which nodes send molecular messages that pass through a molecular channel by means of diffusion. In [28], the authors showed that the molecular channel can be considered Linear and Time Invariant (LTI). Channels subject to this property fulfill the superposition principle, as well as mantain their properties over time. This way, (1) the addition of two received pulses will yield the same signal than the reception of the addition of two pulses; and (2) the channel effects over two independent but identical pulses separated in time will be the same.

This property seems to be crucial for the correct modeling of Quorum Sensing, as this process relies on the accumulation of different emissions of autoinducers. In other words, the superposition principle assures that each node will receive the addition of the emissions of all the other nodes.

\section{Permanent Regime}

The main qualitative reasoning behind the global activation of the bacteria colony is as follows. Generally, the tendency of the molecules is to diffuse from areas of higher concentration to areas of lower concentration [58] (see both Sections 4.1.2 and 5.1). Thus, as time passes, the inhomogeneity in terms of autoinducer concentration decreases, even in presence of punctual emissions. In the end, all the nodes will be sensing a similar concentration and will activate (or not) at a similar time.

Concretely, a considerable amount of time is needed by the autoinducers to diffuse making the concentration almost homogeneous. As it will be seen in the following Sections, the evolution of the particle concentration has two differentiated phases. In the transient phase, the concentration has a strong dependance with time. On the other hand, the concentration reaches a stable value in the permanent regime, as a long enough amount of time has passed.

Cooperative Signal Amplification for Molecular Communication in Nanonetworks 


\section{Perfect Grid Arrangement}

Although in the bacterial world the spatial distribution of bacteria is rather random, Quorum Sensing requires that the nodes are as clustered as possible. To simplify the model and the calculations, the nodes will be arranged in a perfect grid through the three dimensions, forming a cluster. Therefore, the immediate neighbourhood of each node will be the same for all of them. Following this disposition, the node density will be constant over all the space and will only depend on the given distance between adjacent nodes.

Generally, we will consider $\mathrm{N}$ nodes arranged in a cubical space, each of the axis of which will contain $\mathrm{M}$ nodes per axis (see Figure 4.1). Hence, $N=M^{3}$.

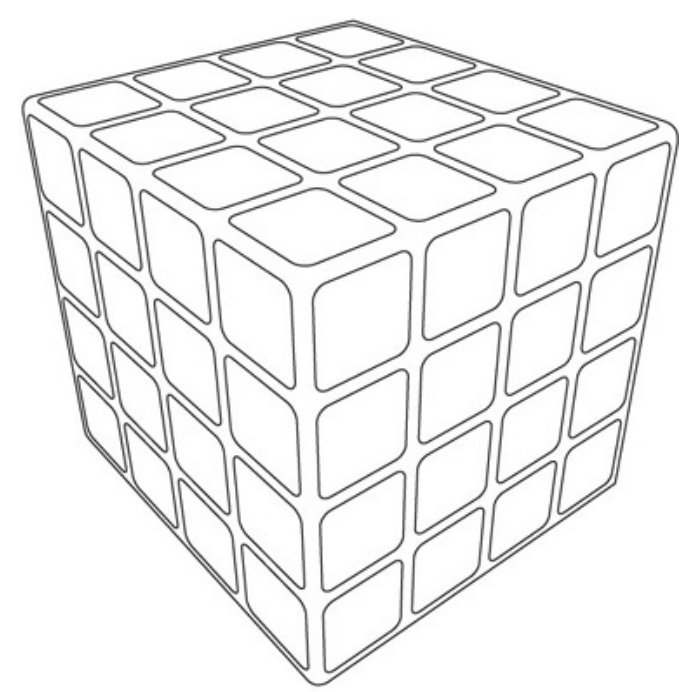

Figure 4.1: Cubic space containing 64 nodes evenly distributed (4x4x4).

\section{Static and Identical Nodes}

The assumptions of permanent regime and perfect grid arrangement imply that nodes will remain static throughout all the process. For that reason, and in spite of the possible flow created by fluids in the environment, or the interaction or collisions of bacteria with particles in their close environment, we consider that bacteria do not move. Flagellated bacteria are known to move in search for nutrients, but the species of bacteria considered in the model do not have flagella. Alternatively, hypothetical nanomachines would not make use of its motility actuators when performing Quorum Sensing. If agents (either bacteria or 
nanomachines) move while performing Quorum Sensing, the distance between them could vary and also the permanent regime of particle concentration might never be reached.

Moreover, we consider that all the agents that perform Quorum Sensing are identical with regard to their model and different parameters (e.g. emission rates). We will assume that the mutation rate, which is present when bacteria replicate their DNA for reproduction, is negligible. There are two aspects that are specially important:

- Sensing Capabilities: an uniformly distributed amount of chemosensors are placed in the surface of the agents that perform Quorum Sensing. Thus, autoinducers coming from any direction will be properly sensed.

- Failure Model: it is known that bacteria can be alive for long periods of time if they have enough nutrients to survive. We will assume that the environment presents enough nutrients to feed the colony during all the simulation time. In the nanomachines scenario, we will consider that energy harvesting systems will provide enough sustenance to complete Quorum Sensing without failing.

\section{Node Transparency}

Quorum Sensing is a phenomenon that involves massive quantities of autoinducers. We believe that considering the nodes that perform Quorum Sensing as transparent nodes is a reasonable assumption. Modeling and computing the collision or binding of the autoinducers with the nodes might be an unfeasible task, taking into account the big number of particles present.

This assumption also implies that autoinducers are destroyed when they are sensed. This way, autoinducers can pass through nodes and be sensed several times while they diffuse away in the medium.

\subsubsection{Diffusion of Autoinducers}

Molecular diffusion, or otherly called simply diffusion, is the thermal motion of all molecules at temperatures above the absolute zero. Following this principle, when in a certain environment exists a non-uniform distribution of particles, these tend to diffuse away in order to

Cooperative Signal Amplification for Molecular Communication in Nanonetworks 
reach an uniform concentration through all the space [58]. Molecular diffusion can be also considered a specific case of random walk or Brownian motion, which models the random movement of particles suspended in a fluid, and also some other phenomena in diverse fields.

The emission and propagation of the autoinducers are subject to these physical rules. When a bacterium emits a certain amount of autoinducers, a peak of concentration appears in a point in space. Then, the autoinducers will diffuse away as explained before, following the gradient of the concentration, therefore going away from the source.

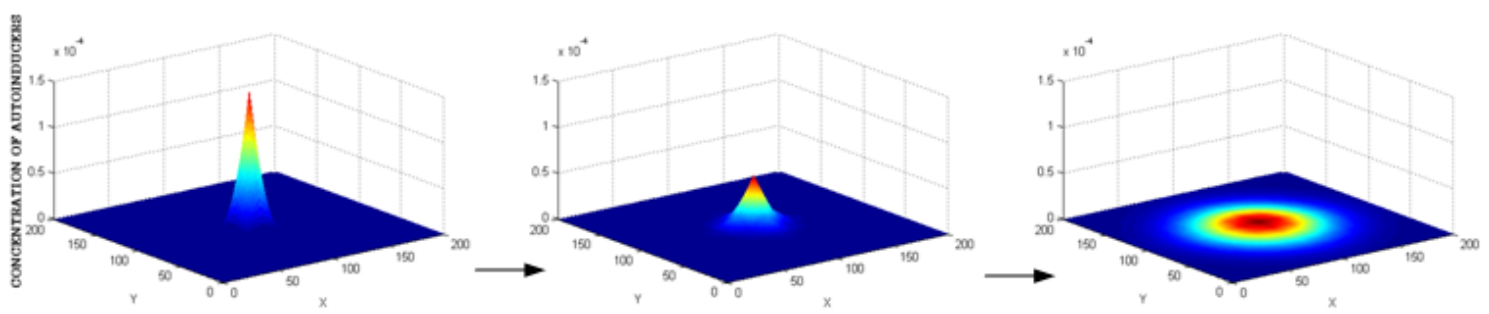

Figure 4.2: Propagation by means of diffusion of a punctual emission.

Quorum Sensing is analytically modeled in this work. The starting point of this model is the equation by Bossert and Wilson [10], which in turn is based on the work of Adolf Fick on molecular diffusion. Hence, it is necessary to explain the Fick's laws of diffusion in order to fully understand the mathematical expressions that will appear in the model.

\subsubsection{Fick's Laws of Diffusion}

Fick's laws of diffusion are, as its own name suggests, mathematical expressions derived by the German physiologist Adolf Fick that describe the diffusion phenomenon. The Fick's first law is as follows:

$$
J(\bar{x}, t)=-D \nabla \phi(\bar{x}, t)
$$

$J$ represents the net flux of particles in a certain n-dimensional position $x$ and time $t$, and depends on the gradient of the concentration of particles $\phi$, and the diffusion coefficient $D$. The gradient, represented by the operator $\nabla$, generalizes the first derivative for ndimensions, being $\nabla \phi(\bar{x}, t)=\left(\frac{\partial \phi(\bar{x}, t)}{\partial x_{1}}, \frac{\partial \phi(\bar{x}, t)}{\partial x_{2}}, \cdots, \frac{\partial \phi(\bar{x}, t)}{\partial x_{n}}\right)$ a vector of the same dimensions that $J$ and $\bar{x}$. 
The diffusion coefficient or diffusivity, gives the "speed" at which the particles move to the positions with less concentration, and is characteristic for each medium. In the Quorum Sensing scenario autoinducers are subject to viscous forces rather than inertial forces, due to the viscosity of the medium, added to the fact that autoinducer particles are nano in size. This can be summarized with the Reynolds Number, which expresses a ratio between inertial and viscous forces. In this case, the fluids in which the autoinducers are spread is considered to be a low Reynolds Number environment, the diffusion coefficient of which, is:

$$
D=\frac{K_{B} T}{6 \pi \eta R}
$$

where $K_{B}$ is the Boltzmann constant, $R$ the radius of the particle, and $T$ and $\eta$ are the temperature and the viscosity of the environment. Considering the medium homogeneous, the coefficient will be a constant for all the points in space. Also, being a key parameter in the modeling of the diffusion, data taken from experiments has been used to determine usual values for different media (see table 4.1 for usual reference values). If not, a first approximation can be done if the viscosity and the expected temperature of the environment are known.

\begin{tabular}{|c||c|c|}
\hline Medium & $\mathbf{T ~}\left({ }^{\mathbf{o}} \mathbf{K}\right)$ & $\mathbf{D}\left(\frac{\mathrm{cm}^{2}}{\mathrm{~s}}\right)$ \\
\hline Air & 298 & {$[0.08-0.8]$} \\
\hline Water & 295 & {$\left[10^{-7}-10^{-4}\right]$} \\
& 310 & {$\left[10^{-10}-10^{-5}\right]$} \\
\hline Blood Plasma & 310 & {$\left[10^{-9}-10^{-7}\right]$} \\
\hline
\end{tabular}

Table 4.1: Orientating values for Diffusion Coefficient.

Fick's second law (equation 4.1.4) predicts how diffusion causes the concentration field to change with time. It is the result of the combination of the first law and the continuity principle. The continuity principle states that particles cannot be created or destroyed, and thus the number of particles entering and leaving the global system must be the same. To express this, the variation of the particle concentration in time (expressed by its derivative $\left.\frac{\partial \phi(\bar{x}, t)}{\partial t}\right)$ must be the opposite of the gradient of the particle flux in the same location $\bar{x}$. 
Hence:

$$
\frac{\partial \phi(\bar{x}, t)}{\partial t}=-\nabla J(\bar{x}, t)
$$

So, substituting the expression of the first Fick's law (4.1.1) into the continuity principle (4.1.3) we obtain the second Fick's law:

$$
\frac{\partial \phi(\bar{x}, t)}{\partial t}=D \nabla^{2} \phi(\bar{x}, t)
$$

where $\nabla^{2}$ expresses divergence of the gradient or sum of the second derivatives. Then, $\nabla^{2} \phi(\bar{x}, t)=\frac{\partial^{2} \phi(\bar{x}, t)}{\partial^{2} x_{1}}+\frac{\partial^{2} \phi(\bar{x}, t)}{\partial^{2} x_{2}}+\cdots+\frac{\partial^{2} \phi(\bar{x}, t)}{\partial^{2} x_{n}}$.

The equation 4.1.4 is the one that will be implemented. It allows us to know the future concentration of particles in one point provided the concentration in one point and its vicinities in the present. Obviously, the autoinducers will be the particles that will diffuse using this principle.

\subsubsection{Finite Differences Method Applied to Fick's Laws}

The second Fick's law can be expressed using the finite differences method in a discrete environment. This methodology is used to model diffusion processes in a discrete environment both in space and time, such as in a simulator. Therefore, we will explain how to obtain the finite differences expression in order to gain insight about how the simulator used in this work operates.

In the aforementioned method, the derivatives are substituted by its approximations by finite differences, thus obtaining:

$$
\frac{\phi(\bar{x}, t+\Delta t)-\phi(\bar{x}, t)}{\Delta t}=D \sum_{i=0}^{n} \frac{\phi\left(\bar{x}-\Delta \overline{x_{i}}, t\right)-2 \phi(\bar{x}, t)+\phi\left(\bar{x}+\Delta \overline{x_{i}}, t\right)}{\left(\Delta \overline{x_{i}}\right)^{2}}
$$

To make the approximations accurate, both differentials of space and time $(\Delta \bar{x}$ and $\Delta t)$ should be small enough to bring them closer to 0 , as in the original definition of derivate expresses $\left(f^{\prime}(a)=\lim _{h \rightarrow 0} \frac{f(a+h)-f(a)}{h}\right)$. Also, the stability of the global equation must be verified, and to accomplish that, the stability equation is used:

$$
\Delta t \leq \frac{(\Delta \bar{x})^{2}}{2 D}
$$

The practical explanation is that, given a spatial resolution and the velocity by which the autoinducers diffuse away, the temporal resolution will be enough to "see" without 
mistake the evolution of all the particles. This condition has to be satisfied wherever the Equation 4.1.5 is applied, like in the N3Sim simulator, which will be presented in the next section.

\subsection{Simulated Environment}

The different insight and assumptions presented so far in this chapter will serve as the basis for the analytical model that will be introduced in Section 5. Those assumptions are generally made for the sake of simplicity, in order to keep the model tractable and the expressions easy to understand. Nevertheless, each assumption represents a layer of abstraction, and distances the model or solution proposed from what in fact happens in real systems.

Simulation is a powerful tool that allows us to implement existing mathematical models. These implementations can be used to validate other analytical models. Simulators are useful because they make use of computational power to overcome complexity-related problems, thus allowing us to relax the original assumptions. In our case, some strong assumptions have been proposed earlier in this chapter and will serve to keep our model tractable; eventually, simulation results will help us to evaluate the performance of the model.

\subsubsection{Existing Simulators}

Several simulators have been created in the recent years, as nanotechnology seems to be the key for upcoming groundbreaking achievements regarding Quorum Sensing.

- In [37], Hense et al show some results based on simulations using a mathematical model of Quorum Sensing. The details are not disclosed, so we cannot assume that the model of bacteria used in this case is similar or different to the one presented in this thesis. Regarding the parameters involved in Quorum Sensing, this simulator uses some approximations for biological data, instead of using values that might be found in the literature. The outcome of the simulations is a nice analysis of the spatial 
clustering and activation of bacteria, concluding that the more together they are, the more effective Quorum Sensing is.

- On the other hand, in [69], some results based in a new simulator are presented. Although it seems that the objective is to give fancy graphic results, Quorum Sensing theory is applied. The model of bacteria used in this simulator has the characteristics of the "mathematical models" described in Appendix A, in the sense that all the enzyme reactions are taken into account. Thus, the computational complexity is high and the simulator does not seem to be capable of dealing with a realistic number of bacteria. In fact, results are shown for populations of only tens of bacteria.

For the reasons here expounded, we will make use of an alternative simulation framework in order to validate the outcome of our model. This simulator is called N3Sim.

\subsubsection{N3Sim}

The N3Sim framework was designed in order to simulate a set of nanomachines which communicate through molecular diffusion in a fluidic medium [46]. This way, N3Sim allows the user to evaluate the communication performance of diffusion-based molecular networks, following the principles explained in Section 1.4.1.1. So far, N3Sim has proved to be a valid simulator, as several consistent results have been presented $[45,28,46]$. In our case, the simulator will be used to validate the results of the analytical model presented in Chapter 5, and to see that the assumptions explained in Section 4.1.1 perform remarkably well.

The diffusion-based molecular communication scenario modeled and simulated by N3Sim can be summarized as follows. The information to be sent by the transmitter nanomachines modulates the rate at which they release molecules, modeled as particles, to the medium. This emission creates variations in the local concentration of particles, which propagate throughout the medium due to the Brownian motion and to interactions among themselves. The receivers are able to estimate the concentration of particles in their neighborhood by counting the number of particles in a volume around their location, called influence area. From this measurement, they can decode the transmitted information [46]. 
The Quorum Sensing scenario can be also simulated by this simulation framework, as it can be regarded as a particular case of diffusion-based molecular communication: several randomly arranged transmitters release particles in the medium, which diffuse away until reaching any of the receivers.

\section{Simulator Architecture}

Figure 4.3 shows a block diagram of the steps needed to run a simulation [46]. The process is as follows:

1. The user specifies first the values of the simulation parameters in a configuration file. These parameters include the number and location of transmitters and receivers, the signal to be transmitted, the size of the emitted particles and the diffusion coefficient of the medium, amongst others. A script file allows the user to run multiple simulations automatically using only one configuration file, which is useful to easily evaluate the influence of a specific parameter (e.g., the number of transmitted particles) in the system output.

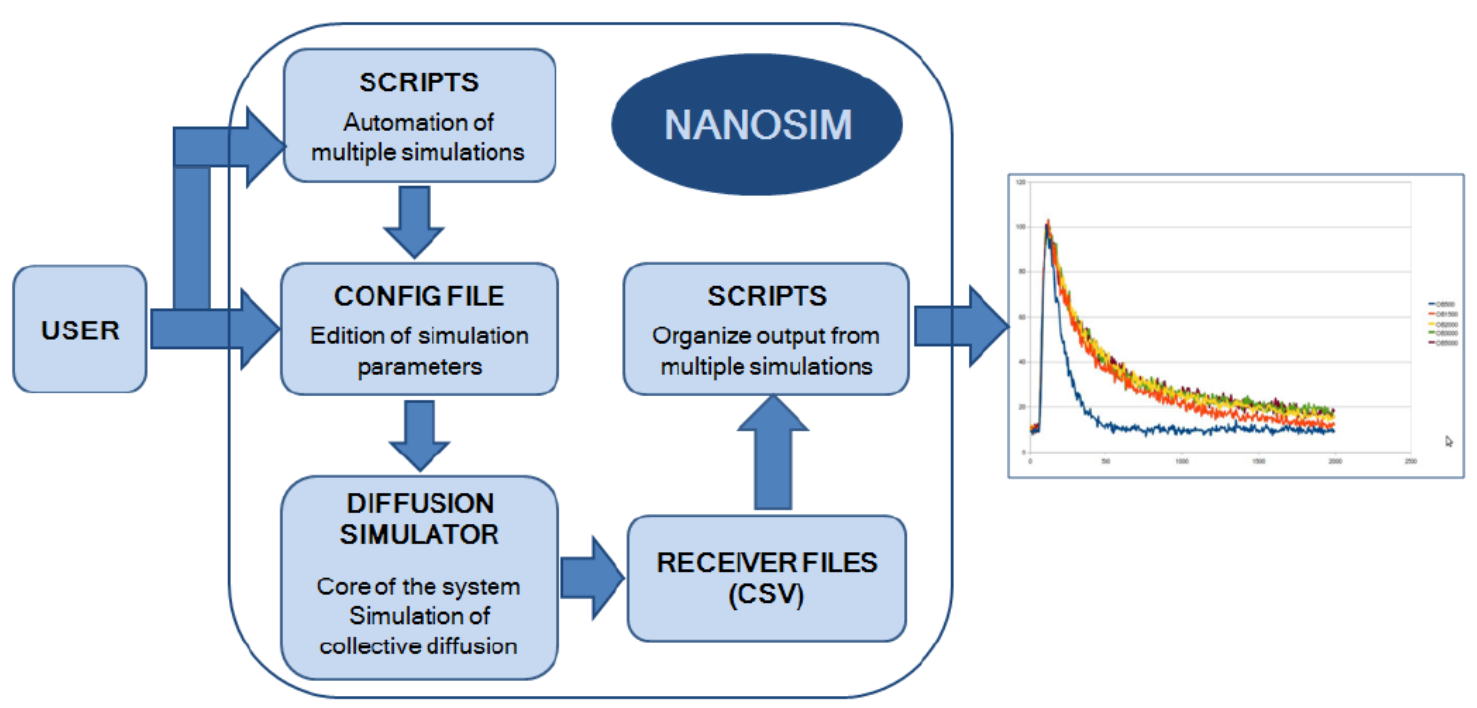

Figure 4.3: Block diagram of N3Sim (from [46]) 
2. Next, the diffusion simulator takes the configuration file and the automation scripts as input, and performs the actual simulation of the diffusion-based molecular communication scenario.

3. When the simulation has ended, its outputs are stored in receiver files (one per receiver), which contain the concentration measured by each receiver as a function of time.

4. Last, another set of scripts may be used to organize the results from several receivers and graphically represent them into a single plot. All the simulation results presented in this work are the outcome of MATLAB scripts that convert text-based receiver files in MATLAB figures.

The configuration file contains all the parameters N3Sim needs to perform the simulations. While a complete list of parameters can be found in [54], we present a summary of the most important ones below:

Basic parameters, summarized in Table 4.2, account for the general variables that apply to all the aspects of the simulation. For instance, the duration and time resolution of the simulation are specified here.

\begin{tabular}{|l||l|l|}
\hline Parameter & Values & Explanation \\
\hline activeCollision & true/false & $\begin{array}{l}\text { Models if physical collisions between particles are consid- } \\
\text { ered. }\end{array}$ \\
\hline inertiaFactor & 0 to 1 & Controls the amount of inertia of the emitted particles. \\
\hline time & integer & Total time of the simulation (in ns). \\
\hline timeStep & integer & Duration of each time step (in ns). \\
\hline
\end{tabular}

Table 4.2: Basic parameters used in the simulator.

Space parameters (Table 4.3) define the characteristics of the simulated environment. For instance, boundedSpace determines if the space is finite or infinite. It is also possible to define the size of the simulated space in case it is finite, or its diffusion coefficient. 


\begin{tabular}{|l||l|l|}
\hline Parameter & Values & Explanation \\
\hline boundedSpace & true/false & $\begin{array}{l}\text { Models if the simulation space is finite or infinite. In the } \\
\text { first case, the size parameters account for the actual size of } \\
\text { the simulation space; on the contrary, if Quorum Sensing } \\
\text { is simulated, they account for the size of cluster in which } \\
\text { the emitters are located. }\end{array}$ \\
\hline xSize & integer & Horizontal size of the simulation space (in nm). \\
\hline ySize & integer & Vertical size of the simulation space (in nm). \\
\hline zSize & integer & If applicable, depth of the simulation space (in nm). \\
\hline D & float & Diffusion coefficient (in $\left.\frac{n m}{n s^{2}}\right)$ \\
\hline sphereRadius & integer & $\begin{array}{l}\text { Radius of the emitted particles, if collisions are taken into } \\
\text { account (in nm). }\end{array}$ \\
\hline
\end{tabular}

Table 4.3: Space parameters used in the simulator.

Emitter parameters (Table 4.4) define the position and characteristics of each one of the emitters included in the simulation. The parameter emitterType is specially relevant as it specifies key features of each emitter. There are up to six different types of emitters: for instance, type-1 emitters execute a constant emission of particles defined by the auxiliar parameter amplitude. Other types of emitters include sources of noise or execute a variable emission of particles. Finally and more importantly for this work, type-6 emitters were specially created to simulate Quorum Sensing, as it allows the deployment of a big number of emitters without having to specify their position.

Similarly to the emitter case, receiver parameters (Table 4.5) define the position and characteristics of each one of the receivers. Again, the parameter receiverType is specially important as it specifies key features of each receiver. In this case, there are three different types of receivers, depending if they are modeled as a square, a circle, or a tridimensional sphere. 


\begin{tabular}{|l||l|l|}
\hline Parameter & Values & Explanation \\
\hline emitters & integer & Number of emitters in the simulation. \\
\hline \multicolumn{2}{|l|}{ Each emitter has the following parameters: } \\
\hline emitterRadius & integer & $\begin{array}{l}\text { Radius of the influence area of the emitter, in which the } \\
\text { emitter is able to sense particles (in nm). }\end{array}$ \\
\hline $\mathrm{x}$ & integer & Horizontal position of the emitter (in nm). \\
\hline y & integer & Vertical position of the emitter (in nm). \\
\hline punctual & true/false & $\begin{array}{l}\text { Determines if particles are emitted exactly at the emitter } \\
\text { location or in randomly in the influence area. }\end{array}$ \\
\hline emitterType & 1 to 6 & $\begin{array}{l}\text { Models different aspects of the emission, such as the pres- } \\
\text { ence of noise, constant or variable emission and if the emit- } \\
\text { ter is tridimensional or not. Depending on the emitter } \\
\text { type, additional parameters have to be defined. }\end{array}$ \\
\hline
\end{tabular}

Table 4.4: Emitter parameters used in the simulator.

\section{Parameter Values}

In this work one of our objectives is to validate the results by simulating molecular communication based on the laws of diffusion. It is known that diffusion takes place as particles collide with each other in a fluidic medium. It would be computationally unfeasible to model each of these collisions individually, since the number of collisions between each particle and the fluid molecules is in the order of $10^{20}$ per second [59]. Therefore, the application of Fick's laws of diffusion is necessary in order to simulate the propagation process. Hence, basic parameters activeCollision and intertiaFactor are set to false and to 0, in order to simulate spontaneous diffusion. It is important to note that as Fick's laws of diffusion are applied, the condition stated in Equation 4.1.6 (Section 4.1.2.2) has to be met in order to ensure the validity of the results.

With regard to space parameters, sphereRadius is set to 0 in concordance with the fact that collisions are not calculated. Moreover, boundedSpace is set to false so that the simulated environment resembles real (not in-the-lab) scenarios. 


\begin{tabular}{|l||l|l|}
\hline Parameter & Values & Explanation \\
\hline receivers & integer & Number of receivers in the simulation. \\
\hline Each receiver has the following parameters: \\
\hline name & string & $\begin{array}{l}\text { Receiver name. The measurements are saved in a CSV file } \\
\text { with this name. }\end{array}$ \\
\hline x & integer & Horizontal position of the emitter (in nm). \\
\hline y & integer & Vertical position of the emitter (in nm). \\
\hline absorb & true/false & Determines if particles are deleted after being sensed. \\
\hline accumulate & true/false & $\begin{array}{l}\text { Determines if the output of the receiver corresponds ei- } \\
\text { ther to the accumulation of particles or to the number of } \\
\text { particles sensed in each timestep. }\end{array}$ \\
& 1 to 3 & $\begin{array}{l}\text { Models the receiver as a square (1), circle (2) or a tridi- } \\
\text { mensional sphere (3). Depending on the receiver type, } \\
\text { additional parameters have to be defined. }\end{array}$ \\
\hline receiverType & &
\end{tabular}

Table 4.5: Receiver parameters used in the simulator.

General considerations about emitters and receivers include the fact that emissions are assumed punctual. On the receiver side, absorb will be set to false in order to take into account the assumption of node transparency in the simulations. The rest of parameters depend on the specific objective of the simulation; we can distinguish two main different types of simulation:

- Point to point: in point to point communication schemes, only one emitter and one receiver are considered. For instance, this situation is simulated in Figure 2.1 to motivate the need of an amplification scheme. In this case, the emitterType parameter is set to 5. Since the scenario for this emitters is tridimensional, we have to define also a $z$ position. The emission pattern of this kind of emitters is determined by a waveform included in the parameter file and multiplied by a scaleFactor. On the receiver side, the parameter receiverType is set to 3 , accordingly to the tridimensional 
scenario. Both the $z$ position and the receiver radius, rradius, have to be defined as well.

- Quorum Sensing: the Quorum Sensing mode of the simulator is activated when the emitterType parameter is set to 6 . In this case, a group of multipleEmitters emitters are randomly arranged in a space located in the origin of coordinates and of size determined by the basic parameters $x$ Size, ySize, zSize. Like in the previous case, the emission pattern of these emitters is defined by a waveform included in a text file. Also, the receivers still are type-3 receivers.

In both cases, the position of emitters and receivers will be defined depending on the objective of the simulation. For instance, in Figure 2.1 we can see the results of two different simulations with different transmission distances in a point to point scheme.

As for biologically-related parameters, we tried to choose values close to the ones shown in experiments or observations, whenever it was possible. For instance, information about the dumping or emission rate of autoinducers is rather scarce throughout the literature. Nevertheless, in [24] the emission rate of amino acids by two marine bacteria is analyzed. These particles are not much different than autoinducers, so we can consider these numbers as a good approximation: from $19 \cdot 10^{-6}$ to $25 \cdot 10^{-6} \mu \mathrm{mol}$ per cell per hour. Values in that range, or below but not far from those numbers, can be therefore assumed. The number of autoinducers released (indicated in the waveform file) and the time between emissions (timeStep) will be selected accordingly.

Values regarding the influence area of the receivers have been chosen taking into account the physical size of bacteria. Bacteria can be as small as tenths of nanometers and as big as almost one millimeter. However, bacteria that apply Quorum Sensing are usually a few microns long, and thus the value assigned to the parameter rradius will be at most in that range. In the same vein, insight found in the literature about the size of bacterial colonies allowed us to calculate the size of our clusters of nanomachines. This way, xSize, ySize and zSize will be selected according to this data in the Quorum Sensing scenario.

Last but not least, the diffusivity or diffusion coefficient determines the speed at which molecules diffuse in the medium. Its value has a direct influence in all the results that will be presented in this work. Fortunately, its value is also very well known for a wide range of 
environments, so that the values used in the simulations will be selected according to the literature. Some examples have been previously shown in Table 4.1.

\section{Scripts}

As previously stated, N3Sim offers the possibility of automating the simulations using scripts, in order to perform several consecutive runs with different parameters. These scripts are programmed in shell scripting and they work as follows. The configuration file allows the passing of parameters from the command line by using the value param. This way, the simulator will scan the configuration file looking for params when starting up. Each of these parameters will have to be added in the command line after the execution command.

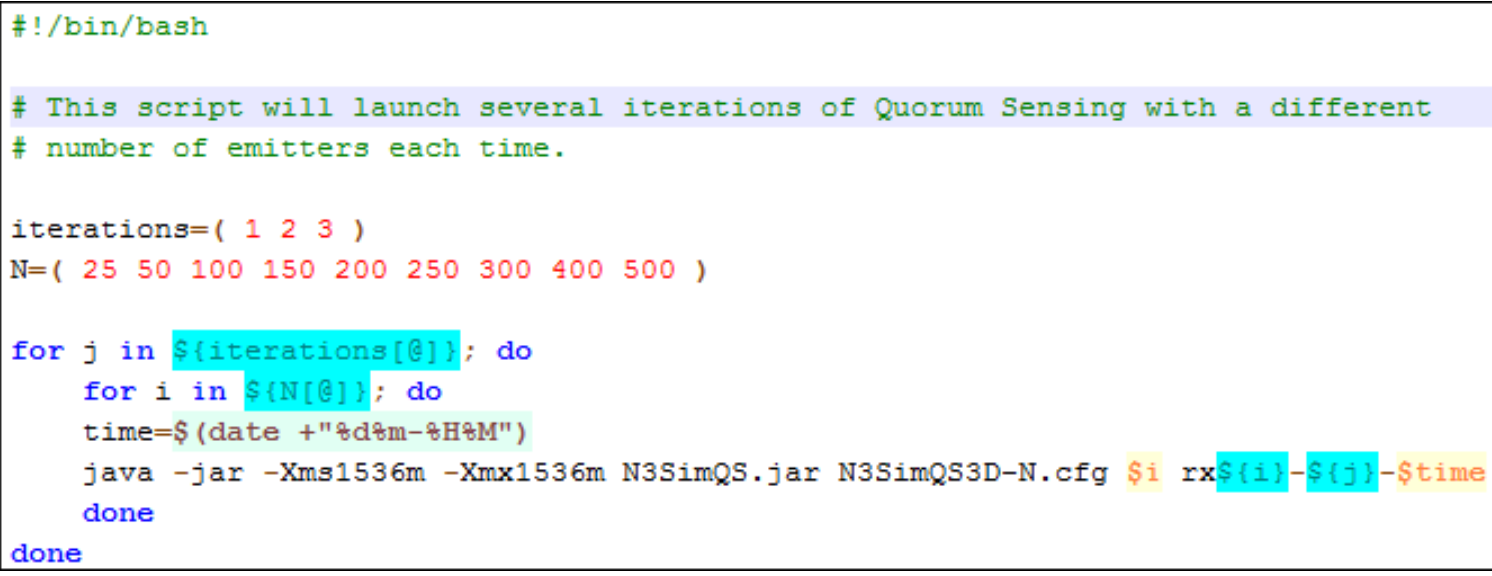

Figure 4.4: Example of a script for several iterations with different number of emitters.

Moreover, the results shown in this work are the result of computing the mean of several repetitions of the same simulation. This is commonly known as the Monte Carlo method. This experiment consists on the application of a deterministic computation on a set of random inputs, and of the aggregation of the results. For instance, let us consider the case of Quorum Sensing simulations. In that case, emitters are deployed randomly in a bounded space. A deterministic computation (i.e. the diffusion process by using the Fick's laws) is performed over the emissions of this group of emitters. This way, we can consider that several repetitions of a deterministic process are run with different random inputs. In 
the end, we calculate the mean of the output of each simulation as a way to aggregate the results.

The objective of following the Monte Carlo method is to improve the performance in terms of accuracy. When using this methodology, the absolute error will decrease proportionally to the square root of the number of repetitions: $\frac{1}{\sqrt{N}}$.

Figure 4.4 shows an example of such scripts, used to extract results for Figures 5.3 and 5.6. The code performs simulations in the Quorum Sensing scenario, varying the number of emitters in each iteration and executing three repetitions for each case. Date and time are also passed as parameters to organize the outputs of the simulation. The configuration file used together with this script had multipleEmitters and name of the receiver as parameters:

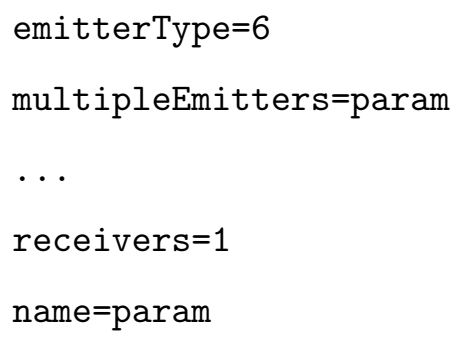




\section{Chapter 5}

\section{Analytical Model and its}

\section{Validation}

In light of the principles explained in Section 3, we can define Quorum Sensing as a process by which a group of agents coordinates its behavior as a function of its population density. Generally, we can consider a given group of agents $\mathbb{S}$ deployed in a certain environment. Each of these agents will release specific synchronization molecules in a constant rate, causing an increase of the molecular concentration in the environment. Finally, these agents are capable of reacting when the concentration of these molecules reaches a certain threshold. While in the biological realm the agents are bacteria and they sense the autoinducer concentration to be aware of their cell density, in our case the agents will be molecular communicationenabled nodes that will use Quorum Sensing to perform cooperative signal amplification.

Indeed, the Quorum Sensing process will serve to synchronize the course of action of a group of $\mathrm{N}$ nodes that will jointly transmit pulses of communication molecules. This cooperative scheme is expected to provide a larger transmission range, as it allows the emission of amplified signals that will be able to reach distant receivers. As we will see in Section 6 , the resultant transmission range will depend on the number of nodes considered.

The objective of this section is to analytically derive the expression of the molecular concentration in a cluster of $\mathrm{N}$ nodes that release molecules at a constant rate, as part of the Quorum Sensing routine. With this expression, we will be able to formally determine 
the mathematical dependence between the concentration of molecules in the environment and the node density. This way, we will extract, as a function of the node count:

1. An estimation of the molecular concentration above which a group of nodes should react (activation threshold).

2. The delay introduced by Quorum Sensing as a synchronization process.

Other models have tried to capture the essence of Quorum Sensing and to extract these results by using mathematical or computational tools. Some examples can be found in Appendix A, as well as the reason why the model here presented is necessary.

In our case we will follow an inductive reasoning. First, in Section 5.1, we will analyze the expression of the molecular concentration given by Bossert and Wilson [10] for a continuous and constant emission of molecules. In Section 5.2, we will derive the molecular concentration resulting from the aggregation of a group $\mathbb{S}$ of emitters, applying the assumptions presented earlier in Section 4.1.1. After that, both the estimation of the activation threshold (Section 5.3) and the delay introduced by the synchronization phase (Section 5.4) will be calculated. All the expressions will be validated through simulation, using the N3Sim framework introduced in the previous chapter.

The notation used in the following sections is summarized in Table 5.1.

\subsection{Individual Emission}

Suppose $Q$ molecules are released by a node at a time $t=0$ in an homogeneous environment of diffusivity $D$. The density $U$ in a point at distance $r$ at a certain instant $t$ is given by Roberts as [10]:

$$
U(r, t)=\frac{2 Q}{(4 \pi D t)^{\frac{3}{2}}} e^{-\frac{r^{2}}{4 D t}}
$$

Taking this equation as a starting point, William H. Bossert and Eduard O. Wilson [10] expressed the density of molecules/autoinducers in the scenario of a constant emission of autoinducers over time. The expression is the following:

$$
U(r, t)=\frac{Q}{4 D \pi r} \operatorname{erfc}\left(\frac{r}{\sqrt{4 D t}}\right)
$$




\begin{tabular}{|l|l|}
\hline Notation & Meaning \\
\hline$r$ & Distance from a given reference point \\
$R$ & Distance from the central node \\
$t$ & Distance between nodes \\
$t_{K}$ & Time instant \\
$U$ & Delay associated to $K$ \\
$K$ & Particle/autoinducer/molecule concentration \\
$k$ & Activation threshold (concentration) \\
$Q$ & Normalized activation threshold \\
$D$ & Emission rate \\
$M$ & Diffusivity or diffusion coefficient \\
$N$ & Mean number of nodes per axis \\
$\rho$ & Total number of nodes $\left(N=M^{3}\right)$ \\
\hline
\end{tabular}

Table 5.1: Summary of the notation used in the model.

where $Q$ now refers to a constant emission rate in molecules per unit of time.

When a node performs a punctual emission, the released molecules diffuse away and the particle concentration in any given point progressively decreases over time. On the contrary, the particle concentration will monotonically increase in the continuous case (the one observed in Quorum Sensing), as the emitter is constantly releasing particles to the medium. However, there is an upper limit for the achievable particle concentration. If the source continues emitting for a long time, the density function will approach the value:

$$
U(r)=\frac{Q}{4 D \pi r}
$$

which will be further used as a normalizing factor.

Figure 5.1 plots the normalized particle concentration over time at a distance of $1 \mu \mathrm{m}$ from the emitter. The theoretic value of the particle concentration becomes independent of time in what can be considered as a permanent regime. Ideally, the flux of molecules 
entering any given volume in this situation is the same as the flux of outgoing molecules, thus stabilizing the value of the particle concentration. Mathematically, this effect is modeled by the asymptotic behavior of the complementary error function, whose Taylor series is:

$$
\operatorname{erf} c(z)=1-\frac{2}{\sqrt{\pi}} \sum_{n=0}^{\infty} \frac{(-1)^{n} z^{2 n+1}}{n !(2 n+1)}=1-\frac{2}{\sqrt{\pi}}\left(z-\frac{z^{3}}{3}+\frac{z^{5}}{10}-\frac{z^{7}}{42}+\cdots\right)
$$

and $z=\frac{r}{\sqrt{4 D t}}$ in our case.

In order to keep the model tractable, we assume that the nodes performing Quorum Sensing release synchronization molecules continuously and that the synchronization is accomplished under permanent regime conditions (see Section 4.1.1). The time value $t$ can be considered to be high, and thus the $z$ term will be close to zero. Hence, under permanent regime, the complementary error function can be approximated as a linear function:

$$
\operatorname{erfc}(z) \approx 1-\frac{2 z}{\sqrt{\pi}}
$$

that can be introduced into Equation (5.1.2). As we can see in Figure 5.1, this approximation performs remarkably well when the time value $t$ is higher than a few milliseconds. The simulation data, obtained by analyzing the concentration sensed by a receiver located 1 $\mu \mathrm{m}$ away of a node emitting at a constant rate, matches both the theoretic value and the approximation.

\subsection{Autoinducers Accumulation}

In the Quorum Sensing scenario, a given number of nodes is deployed randomly forming a cluster. Each of these nodes starts emitting synchronization molecules at some point, at a constant rate. It is possible to calculate the aggregated concentration of molecules at any point in space since:

- The expression of the concentration of autoinducers over time at a distance $r$ of a single transmitter is known, for a constant emission (Eq. (5.1.2)).

- It has been shown in [28] that the molecular channel can be considered Linear and Time Invariant (LTI), and therefore the contributions of the nodes can be aggregated. See Section 4.1.1 for more details about this feature. 


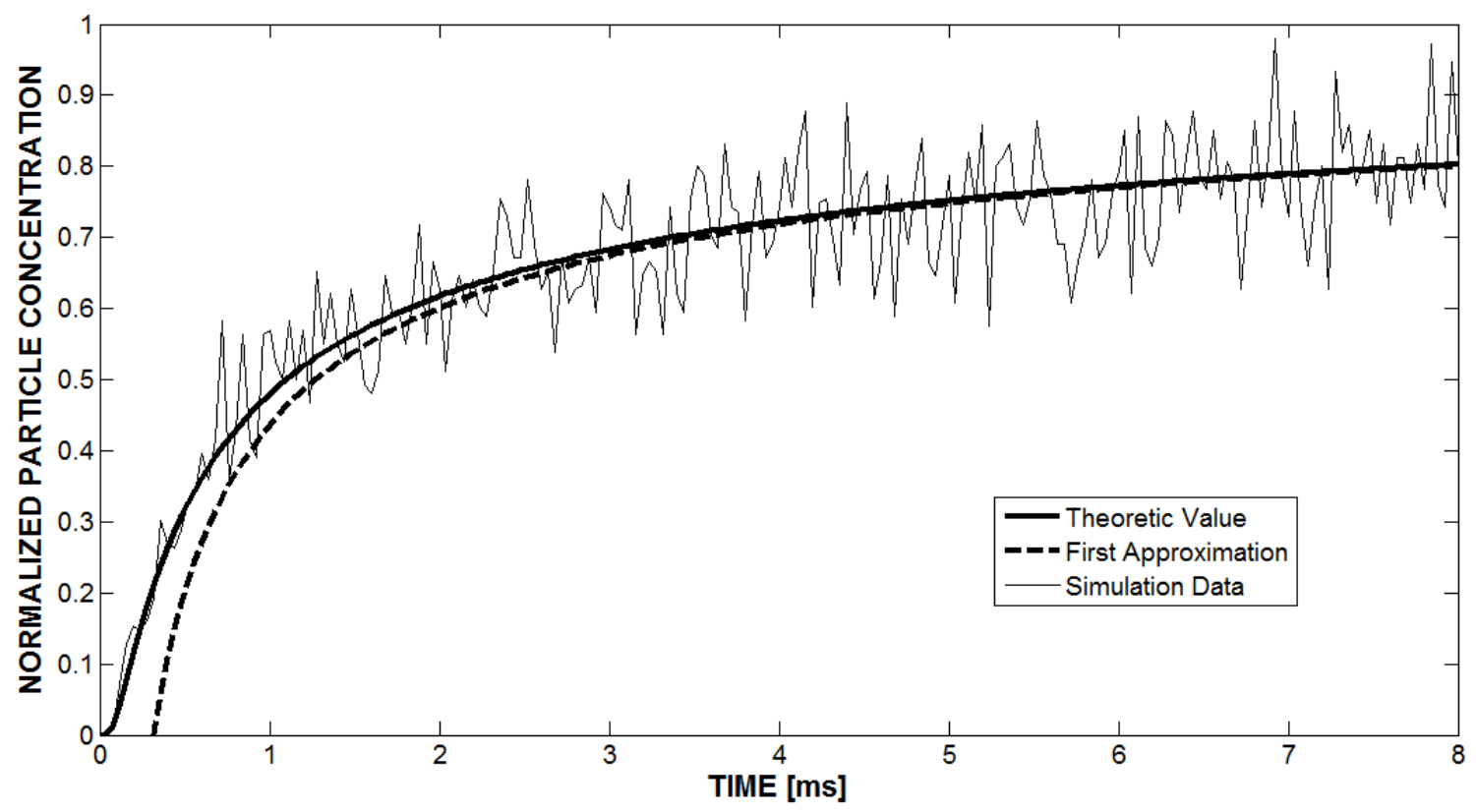

Figure 5.1: Simulation data, theoretic value and first approximation of the normalized particle concentration at a distance of $r=1 \mu m$ of a constant emitter

Then, the concentration of molecules at a specific point is the sum of the contributions of a group $\mathbb{S}$ of emitters. As discussed earlier, we consider that all nodes are identical (same emission rate). In this case, each emitter is at a distance $r_{i}(i \in \mathbb{S})$ of the evaluated point, so that if we use the value of the maximum achievable concentration shown in Equation (5.1.3), we obtain:

$$
U=\sum_{i \in \mathbb{S}} \frac{Q}{4 D \pi r_{i}}=\frac{Q}{4 D \pi} \sum_{i \in \mathbb{S}} \frac{1}{r_{i}}
$$

Even though the nodes are expected to be randomly deployed, we assume that the nodes are arranged in a perfect tridimensional grid so that the immediate neighbourhood of each node will be the same for all of them (see Section 4.1.1). This assumption helps to keep the model tractable without compromising its accuracy. The analytical results will be compared with simulation data in which the nodes are randomly arranged, in order to show that the approximation performs well enough.

Following the perfect grid disposition, the node density $\rho$ will be constant over all the space and will only depend on the given distance between adjacent nodes $R$. For instance, a 


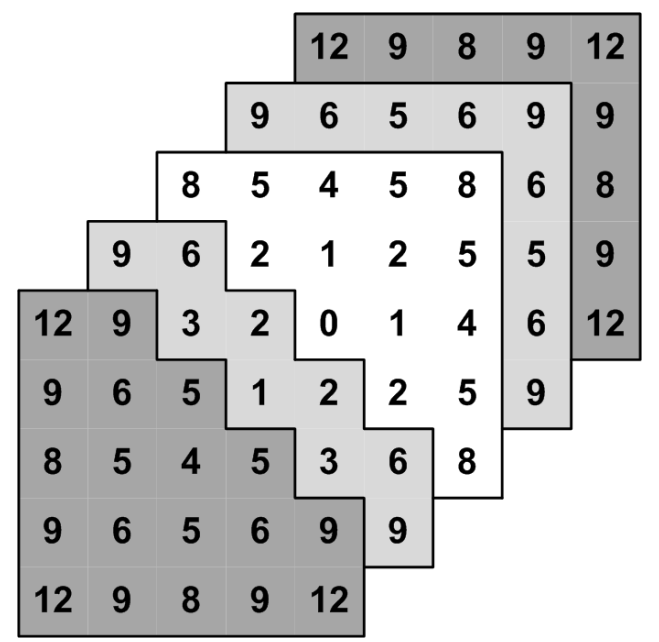

Figure 5.2: Squared normalized distances with respect to the central node, in a perfect tridimensional grid of five units of arista.

cube of $(M R)^{3}$ volume units will contain $N=M^{3}$ cells and thus, the resulting node density will be:

$$
\rho=\frac{M^{3}}{(M R)^{3}}=\frac{1}{R^{3}}
$$

Moreover, the euclidean distance between any given two nodes of the grid will be a proportional to $R$ (check Figure 5.2 for an example). In a perfect grid of $\mathrm{N}$ nodes (MxMxM), we can then calculate the particle concentration sensed by a node situated at any given point. For a node located at $\left(X_{d}, Y_{d}, Z_{d}\right)$, at an euclidean distance $d$ of the central node $\left(X_{0}, Y_{0}, Z_{0}\right)$, the particle concentration can be expressed as:

$$
U(N, d)=\frac{Q}{4 D \pi} \sum_{i \in \mathbb{S}} \frac{1}{r_{i}}=\frac{Q}{4 D \pi R} \Phi(N, d)
$$

where $\Phi(N, d)$ stands for the sum of the inverse of the normalized distances between the considered node and all the other nodes of the cluster:

$$
\Phi(N, d)=\sum_{i} \sum_{j} \sum_{k} \frac{1}{\sqrt{\left|X_{i}-X_{d}\right|^{2}+\left|Y_{j}-Y_{d}\right|^{2}+\left|Z_{k}-Z_{d}\right|^{2}}}
$$

Indexes $i, j$ and $k$ range from $-M / 2$ to $M / 2\left(N=M^{3}\right)$ but without including the index corresponding to position $\left(X_{d}, Y_{d}, Z_{d}\right)$, and represent relative positions with respect to 
the central node, for each cartesian axis. We can further treat this equation by introducing $\Phi(N, 0)$ or "central aggregation factor", which accounts for the sum of the contributions of the $\mathrm{N}$ nodes to the central molecular concentration:

$$
U(N, d)=\frac{Q}{4 D \pi R} \Phi(N, d) \frac{\Phi(N, 0)}{\Phi(N, 0)}
$$

The resulting $\frac{\Phi(N, d)}{\Phi(N, 0)}$ term will be referred as attenuation factor or $\alpha(d)$, whereas $\Phi(N, 0)$ can be simply expressed as $\Phi(N)$. Therefore:

$$
U(N, d)=\frac{Q}{4 D \pi R} \alpha(d) \Phi(N)
$$

Note that a summary of the expression and the physical meaning of these non-elemental factors (i.e. $\Phi(N)$ and $\alpha(d)$ ) can be found in Table 5.2.

Finally, introducing the density equation (5.2.2) into Eq. (5.2.6) we obtain:

$$
U(N, d)=\frac{Q}{4 D \pi} \rho^{1 / 3} \alpha(d) \Phi(N)
$$

This last expression is quite intuitive and proves how the molecular concentration $U$ is proportional to the node disposition, which is modeled by the total number of nodes deployed $N$ and the node density $\rho$. While the molecular concentration is directly proportional to the node density, its dependence with the total number of nodes is modeled through the central aggregation factor $\Phi(N)$ (see Figure 5.3).

Therefore, a certain number of nodes $N$ will need to be deployed forming a cluster of density $\rho$ in order to reach a threshold concentration of autoinducers and thus to activate the whole colony, following the Quorum Sensing principles. The achievable concentration also depends on the nodes characteristics (emission rate $Q$ ) and the environment in which they are deployed (diffusion coefficient $D$ ).

Nevertheless, the concentration is not homogeneous as it depends on the distance $d$ to the central node. Specifically, the concentration slightly decreases as we approach the edges of the cluster. This centrality dependence of the molecular concentration is modeled by the position-related attenuation factor $(\alpha(d))$, the behavior of which is shown in Figure 5.4. The attenuation is low and almost constant at the core nodes, reaching only significant values in outer areas and a maximum attenuation of no more than $3 \mathrm{~dB}$ at the edge. 


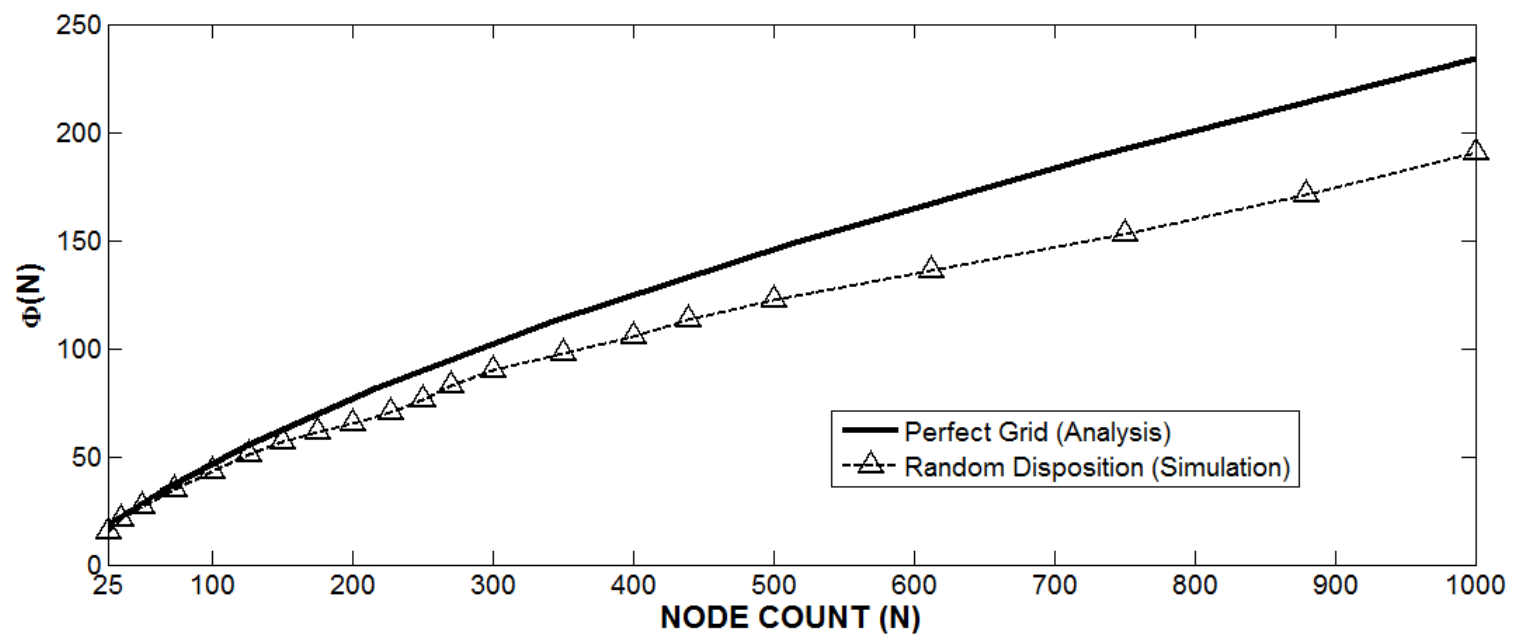

Figure 5.3: Central aggregation factor $\Phi(N)$ as a function of the number of nodes $N$.

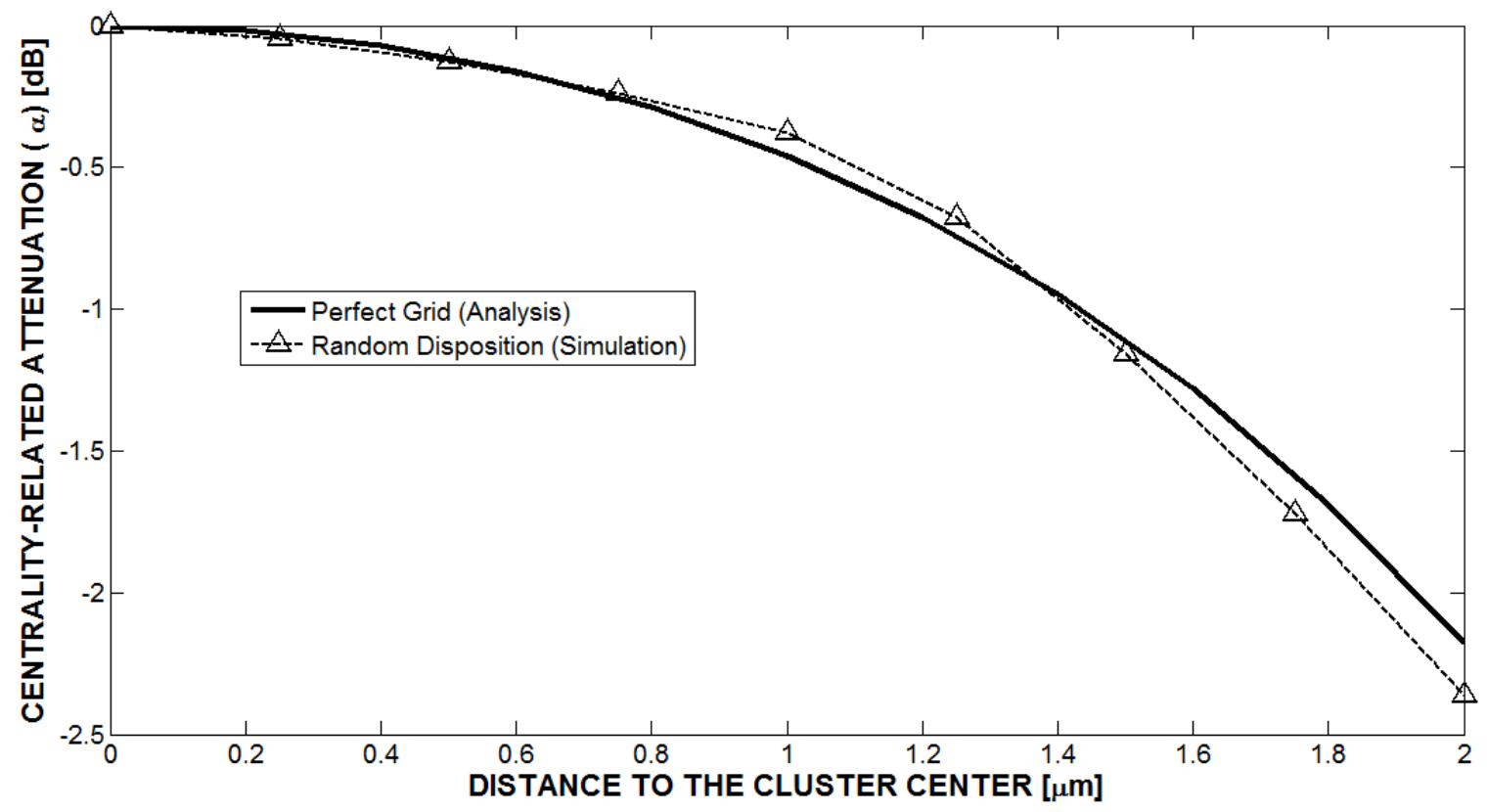

Figure 5.4: Position-dependent attenuation factor $\alpha$ as a function of the distance to the central node $d$ in a cluster of radius $2 \mu \mathrm{m}$.

Finally, it is worth pointing out that the perfect node arrangement approximation performs remarkably well in light of the results shown in Figures 5.3 and 5.4, in which the theoretic values do not differ much from the simulation data. 


\subsection{Threshold Calculation}

As previously discussed, nodes performing Quorum Sensing react upon sensing a concentration of autoinducers higher than a given level called activation threshold. These nanomachines will be randomly deployed in the targeted environment and are expected to be identical, thus having the same activation threshold. The choice of a reasonable value for this parameter is the key for the proper activation of nanodevices in the Quorum Sensing phase. For instance, the synchronization process will obviously fail if the activation threshold is set above the maximum achievable molecular concentration. Therefore, the threshold $K$ is should be lower than the value of $U_{\max }$ :

$$
K<U_{\max }=\frac{Q}{4 D \pi} \rho^{1 / 3} \Phi(N)
$$

which is, under the assumption of a perfect tridimensional grid, the concentration sensed at the central node.

Equation (5.3.1) sets the maximum value for the activation threshold in a cluster of node density $\rho$ consisting of $N$ nodes, for a specific set of emitters in a certain environment. In order to abstract the threshold discussion from these parameters, we will introduce the normalized threshold:

$$
k=\frac{K}{U_{\max }}
$$

which will be a number in the range $k \in(0,1)$.

A node will activate if the molecular concentration surpasses the activation threshold, or $U>K$. If we apply this inequality to the Equation 5.3.2, we obtain:

$$
k<\frac{U}{U_{\max }}=\alpha(d)
$$

This last expression means that nodes which the activation threshold is lower than the position-related attenuation they suffer. In other words, nodes located within a certain range from the central node $(d)$ will activate. The molecular concentration depends on the position of each node inside the cluster, as shown in the previous section. More specifically, the concentration decreases as we approach the edges of the cluster, phenomenon that is modeled by the $\alpha(d)$ factor (see Figure 5.4). Therefore, the activation threshold determines the percentage of nanodevices of a cluster that will effectively synchronize: in corcondance 


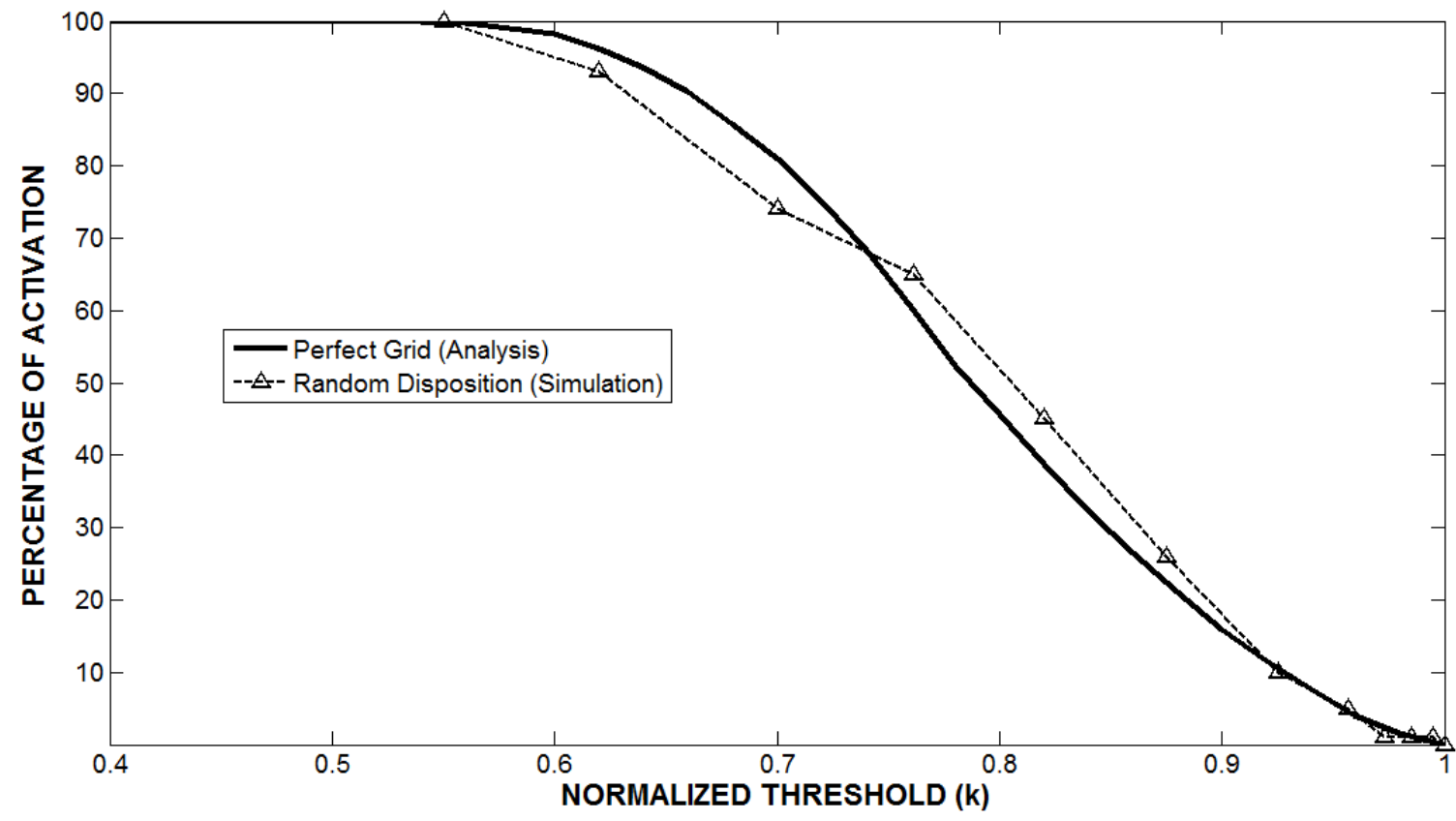

Figure 5.5: Percentage of activation of a cluster of radius $2 \mu \mathrm{m}$ as a function of the chosen threshold.

with the last result, a low threshold should ensure an activation of $100 \%$ of the nodes of a given transmission cluster.

Figure 5.5 shows the evolution of the percentage of activation as a function of the relative activation threshold, using the data of the attenuation factor shown in Figure 5.4. Indeed, we can observe that low thresholds ensure the activation of a big number of nodes, whereas the percentage of activation rapidly decreases when the threshold is raised. It is interesting to note that a threshold of approximately $55 \%$ of the maximum achievable concentration $(k=0.55)$ ensures the total activation of the cluster. Thresholds below that value will also enable a total activation of the group of nanomachines and, as we will see in the next section, will result in a lower delay.

Nevertheless, chosing an excessively low threshold in order to achieve $100 \%$ compromises the quality of the transmission. As we will see in Section 6, the number of synchronizing nodes determines the maximum distance at which a message will be successfully decoded. Transmission clusters consisting of less than a certain number of nodes will not be able to reach receivers located at a given distance and should not activate due to molecular 
interference and energy consumption reasons. However, these clusters will still activate if the preset threshold is inappropriate.

\subsection{Delay Calculation}

Once the activation threshold $K$ for Quorum Sensing-enabled nodes is chosen, an approximation of the time needed to reach quorum can be calculated. If we use the first approximation shown in Equation (5.1.5), the concentration of autoinducers in a certain point in space can be expressed as:

$$
U(r, t) \approx \frac{Q}{4 D \pi} \sum_{i \in \mathbb{S}}\left[\frac{1}{r}-\frac{1}{\sqrt{\pi D t}}\right]
$$

Using Eq. (5.2.7), for a set consisting of a perfect grid of $\mathrm{N}$ nodes, we can obtain:

$$
U(N, d, t) \approx \frac{Q}{4 D \pi}\left[\rho^{1 / 3} \alpha(d) \Phi(N)-\frac{N}{\sqrt{\pi D t}}\right]
$$

Ideally, the nodes will activate when the concentration $U$ reaches or surpasses the threshold $K$. The time needed for this to happen $t_{K}$ can be approximately obtained from Equation (5.4.2), when $U=K$.

$$
t_{K}(d) \approx \frac{Q^{2}}{16 D^{3} \pi^{3}} \frac{N^{2}}{\left(U_{\max } \alpha(d)-K\right)^{2}}
$$

As previously stated, the threshold $K$ has to be necessarily below the maximum molecular concentration $U_{\max }$. If we suppose that the threshold will be $K=k \cdot U_{\max }$, with $k \in(0,1)$, the delay can be expressed as follows:

$$
t_{K}(d) \approx \begin{cases}\frac{1}{D \pi}\left(\frac{1}{\rho^{1 / 3} \Phi^{\prime}(N)}\right)^{2} \frac{1}{\Delta U(d)^{2}} & k<\alpha(d) \\ \infty & k \geq \alpha(d)\end{cases}
$$

where $\Delta U(d)=\alpha(d)-k$ will be further referred as "concentration margin". Also, the dependence with the number of nodes $N$ is solely modeled with the term $\Phi^{\prime}(N)=\frac{\Phi(N)}{N}$, which accounts for the mean contribution of each node to the total molecular concentration. Remember that these derivative expressions can be found in Table 5.2 together with their physical meaning. 
The approximate delay introduced by the synchronization process is given by Equation (5.4.4), being known the diffusivity $D$, the total number of nodes $N$ deployed with a density $\rho$, and the concentration margin $\Delta U$. The delay is infinite in those areas in which the threshold is higher than the maximum achievable concentration, or $k>\alpha(d)$.

\begin{tabular}{|c|c|c|}
\hline Variable & Name & Expression \\
\hline$\Phi(N, d)$ & Aggregation factor & See Eq. (5.2.4) \\
\hline \multicolumn{3}{|c|}{$\begin{array}{l}\text { Meaning: Sum of the contributions of } N \text { nodes to the molecular } \\
\text { concentration at a point at distance } d \text { from the central node }\end{array}$} \\
\hline$\Phi(N)$ & Central aggregation factor & $\Phi(N, 0)$ \\
\hline \multicolumn{3}{|c|}{$\begin{array}{c}\text { Meaning: Sum of the contributions of } N \text { nodes to the molecular } \\
\text { concentration at the central node }\end{array}$} \\
\hline$\Phi^{\prime}(N)$ & Mean individual contribution factor & $\frac{\Phi(N)}{N}$ \\
\hline \multicolumn{3}{|c|}{$\begin{array}{l}\text { Meaning: Mean contribution of each of the } N \text { nodes to the central } \\
\text { molecular concentration }\end{array}$} \\
\hline$\alpha(d)$ & Attenuation factor & $\frac{U(N, d)}{U_{\max }(N)}$ \\
\hline \multicolumn{3}{|c|}{ Meaning: Attenuation at a point at distance $d$ from the central node } \\
\hline$\Delta U(d)$ & Concentration margin & $\alpha(d)-k$ \\
\hline Meaning: D & $\begin{array}{l}\text { fference between the maximum concent } \\
\text { distance } d \text {, and the chosen thresho }\end{array}$ & ation achievable at \\
\hline
\end{tabular}

Table 5.2: Summary of the non-elemental variables used in the model.

As the previous equation suggests, the delay is highly determined by the node distribution. The more densely clustered the nodes are deployed, the higher the $\rho$ factor will be, thus improving the delay performance. This seems consistent with the intuitive explanation: the distance between nodes will be shorter if they are densely deployed, thus reducing the time needed for the molecular concentration to reach a certain level. The node count $N$ also has influence upon the delay through the inverse of the mean individual contribution $\Phi^{\prime}(N)$. Figure 5.6 shows that the contribution of each node to the overall molecular concentration diminishes as the node count grows, making the delay to increase accordingly. 


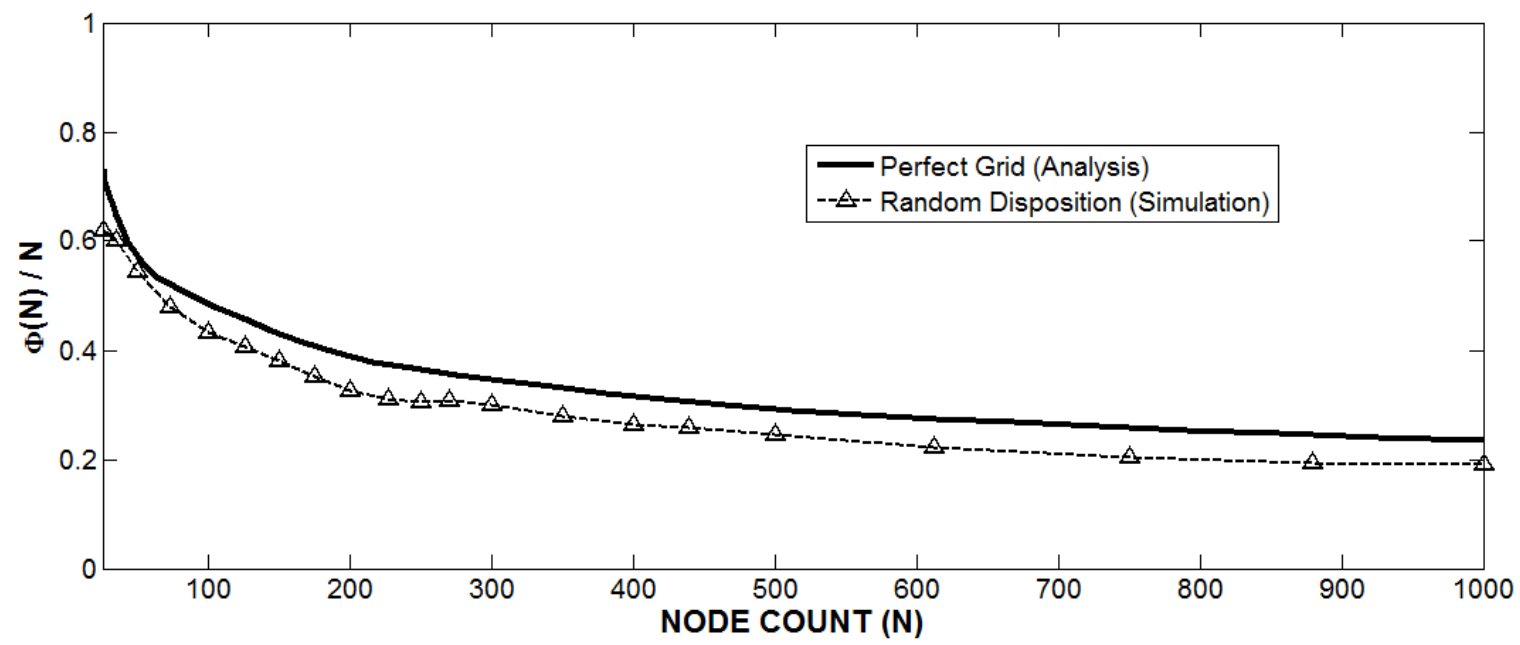

Figure 5.6: Mean individual contribution factor $\Phi^{\prime}(N)=\frac{\Phi(N)}{N}$ as a function of the number of nodes $N$.

Secondly, the medium in which the nodes are deployed also affects the delay figures. In particular, the delay is inversely proportional to the diffusion coefficient $D$, as this parameter models how fast molecules diffuse away.

Finally, the delay depends on the activation threshold $K$ through the concentration margin term. In particular, the delay is inversely proportional to the square of the concentration margin $\Delta U(d)$, which represents the difference between the maximum attainable molecular concentration at a distance $d$ of the cluster center, and the chosen threshold level. Therefore, the delay is a stochastic process that is dependent on the position of the considered node.

The delay will be short as long as the concentration margin is high. In other words, the delay will be short as long as:

- The maximum attainable concentration is high. The delay will always be lower in central positions than closer to the edge of the cluster, as the molecular concentration decreases with distance (Figure 5.4). This effect explains the spatial dependence of the delay. 


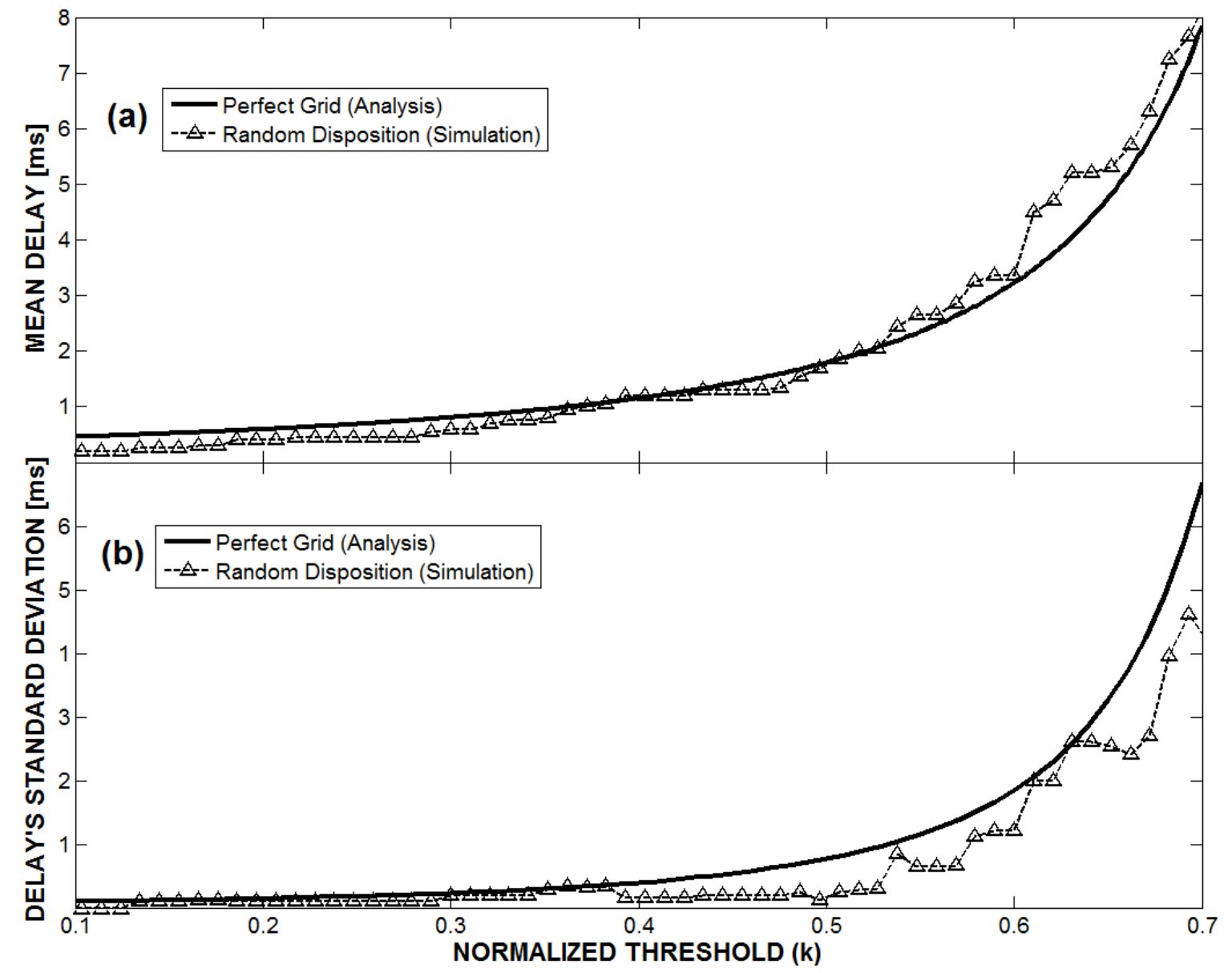

Figure 5.7: Synchronization mean delay $E\left[t_{k}(d)\right]$ and standard deviation $\sigma\left[t_{k}(d)\right]$ as a function of the chosen threshold.

- The threshold is set to a low value. Figure 5.7 shows how lower thresholds imply shorter delays, due to the fact that the sensed molecular concentration needs less time to reach the activation level.

Indeed, the results shown in Figure 5.7 could lead to the conclusion that the activation threshold has to be set to a low value so that the delay is minimized both in terms of mean value and variance. Nevertheless, an excessively low threshold compromises the quality of the transmission. As introduced in Section 5.3, the threshold has to be chosen as a function of the transmission range desired instead of the delay. Eventually, a higher transmission range implies the need of a higher threshold which, in turn, entails a larger delay. 


\section{Chapter 6}

\section{Amplification}

Recalling from Section 2, the amplitude of molecular pulses decreases proportionally to the third power of the transmission distance [45]. Thus, reaching certain distances using molecular communication schemes might result unfeasible, due to the energy constraints inherent to nanomachines. However, by using Quorum Sensing, a group of nodes could coordinate their actions to transmit the same pulse synchronously. This way, the resultant pulse will be effectively amplified and the transmission range of the system will be significantly improved.

Summarizing, the process of cooperative emission would have two phases:

1. Synchronization Phase: the principles of Quorum Sensing are applied in this phase. The original transmitter starts emitting autoinducers at a constant rate. Adjacent nodes detect these synchronization molecules and start emitting molecules of the same type until its concentration surpasses the activation threshold. Eventually, a transmission cluster of approximately $N_{E}$ nodes activates at a similar time instant.

2. Amplification Phase: the cooperative actuation occurs in this phase. The group of nodes has successfully performed Quorum Sensing and proceeds to the emission of a pulse of communication molecules. As the molecular channel is additive, the resultant pulse would be received as the sum of the different pulses. The pulse information could be encoded in the autoinducers used in the synchronization phase, in order to guarantee that all the nodes will transmit the same signal. 


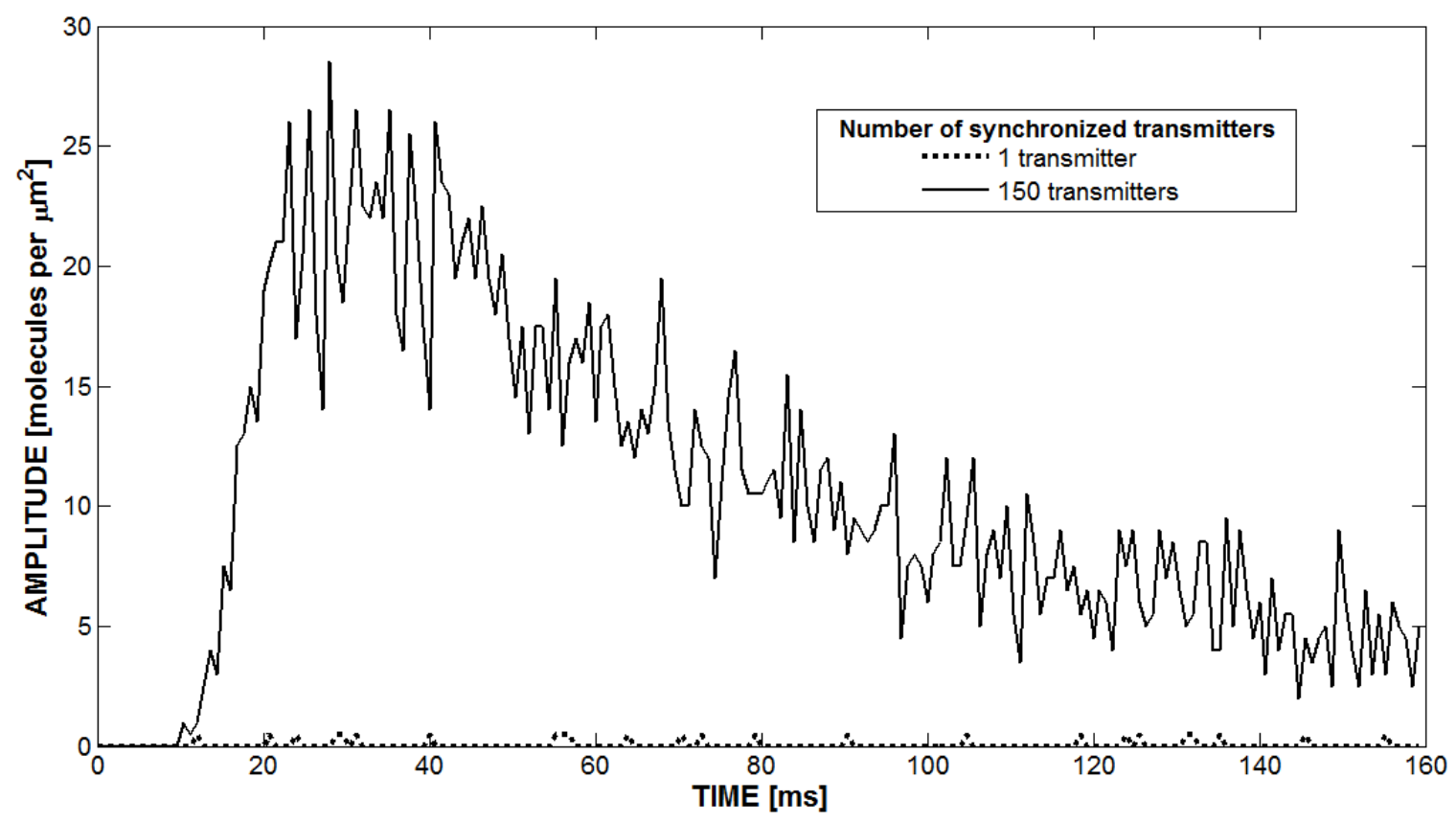

Figure 6.1: Reception, at a distance of $50 \mu \mathrm{m}$, of a pulse emitted by one transmitter (dotted line) and of a pulse amplified by a group of 150 transmitters (solid line).

The model presented in Chapter 5 provides two fundamental expressions for the synchronization phase: activation threshold for a transmission cluster of $N_{E}$ nodes -Eq. (5.3.1)and the delay introduced by this phase -Eq. (5.4.4)-. Alternatively, this section will focus on the amplification phase, more concretely on determining the improvement achieved in terms of transmission range.

We performed two different simulations in which a single 2-millisecond long pulse is transmitted to a receiver located at a distance of 50 micrometers from the transmitter area. The pulse is transmitted by a single emitter in the first simulation, whereas a group of 150 emitters is the transmission source in the second simulation. Each emitter has an identical transmission power of 250 molecules per microsecond in either case. Figure 6.1 shows the evolution over time of the particle concentration received in these two different simulations. The receiver was only able to sense residual concentration of particles in the case of individual transmission, while the amplified pulse can be clearly identified in the second case. Eventually, the distant receiver will be able to successfully decode the molecular pulse if there is a signal amplification at the source. 


\subsection{Transmission Range}

In [45], the amplitude of a molecular pulse in reception is calculated as the molecular concentration at the time instant at which the pulse reaches its maximum value, yielding:

$$
c_{\max }=\left(\frac{3}{2 \pi e}\right)^{\frac{3}{2}} \frac{Q}{r^{3}}
$$

If the receiver is far enough of a cluster of $N_{E}$ transmitters that transmit $Q$ particles each, we can consider the group as a punctual emitter that is releasing $N Q$ communication molecules. If the receiver is at a distance $r$ and has a sensitivity of $c_{S}$, the number of emitters needed $N_{E}$ can be calculated by solving the equation $c_{\max , N}>c_{S}$ :

$$
N_{E}>\frac{c_{S} r^{3}}{Q}\left(\frac{2 \pi e}{3}\right)^{\frac{3}{2}}
$$

Hence, a transmission cluster of $N_{E}$ nodes emitting $Q$ molecules each, will be correctly received if a receiver of sensitivity $c_{S}$ molecules per volume unit is within a distance of:

$$
r<r_{\max }=\sqrt{\frac{3}{2 \pi e}}\left(\frac{N_{E} Q}{c_{S}}\right)^{\frac{1}{3}}
$$

and thus defining the transmission range $r_{\max }$.

Figure 6.2 shows how the normalized transmission range scales as the number of synchronized emitters increases, as well as the behavior of its first order derivative. Specifically, the transmission range is proportional to the cube root of the number of emitters used, and both figures evidence a certain saturation of the amplification when the number of nodes is high. Either way, the final transmission range will depend on the range of an isolated emitter, which can be approximately calculated by using Eq. (6.1.3) with $N_{E}=1$.

While it is relatively easy to find experimental data about pheromone release rates and odor receptors sensitivity in the macroscale (in [11], for instance), data about molecular secretion rate of unicellular agents in aqueous environments is rather scarce. Nevertheless, we found out that Dictyostelium discoideum, an amoebae species, is capable of secreting a certain type of molecule in a rate of approximately 120 units per second [17].

As for molecular sensitivities, a concentration between 5 and $10 \mathrm{nM}$ (nanomols per liter, $6.022 \cdot 10^{11}$ molecules per $\mathrm{cm}^{3}$ ) autoinducers is enough to activate certain genes in 

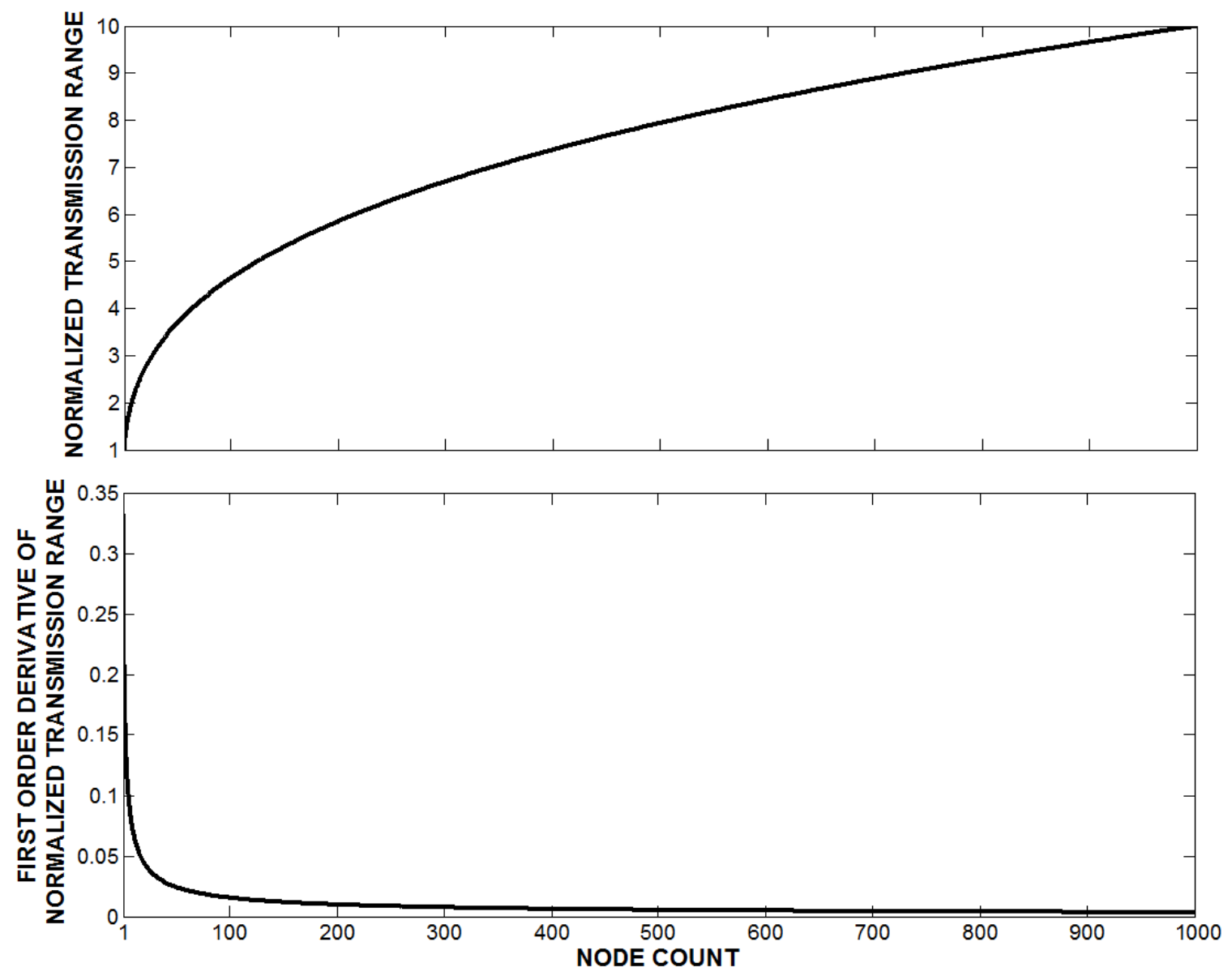

Figure 6.2: Normalized transmission range and its first order derivative as a function of the number of nodes used to amplify the signal.

Vibrio fischeri or Escherichia coli [20,26]. Also, receivers of certain fungi are able to react to concentrations in the picomolar range [77].

Then, let us consider a transmitter capable of emitting 120 molecules per second [17] and a receiver with a sensitivity of 10 picomols [77] (equivalent to a concentration of 6.02 . $10^{-3}$ molecules per $\left.\mu \mathrm{m}^{3}\right)$. Under these conditions, the transmission range when emitting a 1-second pulse, will be:

$$
r_{\max }=\sqrt{\frac{3}{2 \pi e}}\left(\frac{120}{6.02 \cdot 10^{-3}}\right)^{\frac{1}{3}}=11.36 \mu \mathrm{m}
$$

Eventually, the transmission range can be enhanced by the cooperative amplification method depicted in this paper. For instance, a group of 125 emitters could reach distances of over 
$50 \mu \mathrm{m}$, whereas a group of 1000 emitters could be successfully received more than $100 \mu \mathrm{m}$ away from the source.

\subsection{Threshold Election}

The connection between the model introduced in Chapter 5 for the synchronization process and the results here presented for the amplification process stems on the fact that the election of a certain threshold implicitly sets the level of amplification and, in turn, the final transmission range of the system.

Indeed, the level of amplification can be variable and could depend on the transmission distance needed at a certain moment. Actually, for given a set of emitters, we could achieve a variable transmission range by varying the activation threshold of the cluster.

The explanation is as follows. Equation 6.1.2 shows an expression of the number of emitters $N_{E}$ needed to reach a transmission distance $r$, depending on several other parameters. We can consider, for energy efficiency reasons, that we are interested in only activating the minimum number of emitters to reach a distance. Therefore, $N_{E}$ should be the lower bound of the aforementioned equation:

$$
N_{\text {min }}=\left\lfloor N_{E}\right\rfloor=\left\lfloor\frac{c_{S} r^{3}}{Q}\left(\frac{2 \pi e}{3}\right)^{\frac{3}{2}}\right\rfloor
$$

If we want to achieve a certain transmission distance $r$ we will need $N_{\min }$ synchronized transmitters. In order to activate at least $N_{\min }$ transmitters from a cluster of a total of $N$ deployed nanomachines we can make use of the empyric results stemming from the expression $k<\alpha(d)$. That expression led to the Figure 5.5 which shows the percentage of activation depending of the chosen activation threshold. Thus, we can conclude that the chosen threshold determines the number of nanomachines that will activate and therefore the amplification and final transmission range of the system.

Figure 6.3 shows the aforementioned connection between the activation threshold and the final accomplished amplification level. Indeed, low activation thresholds imply the activation of a high percentages of the nodes and thus, higher amplification levels. On the other hand, we might prefer to achieve a lower amplification level by activating a lower 


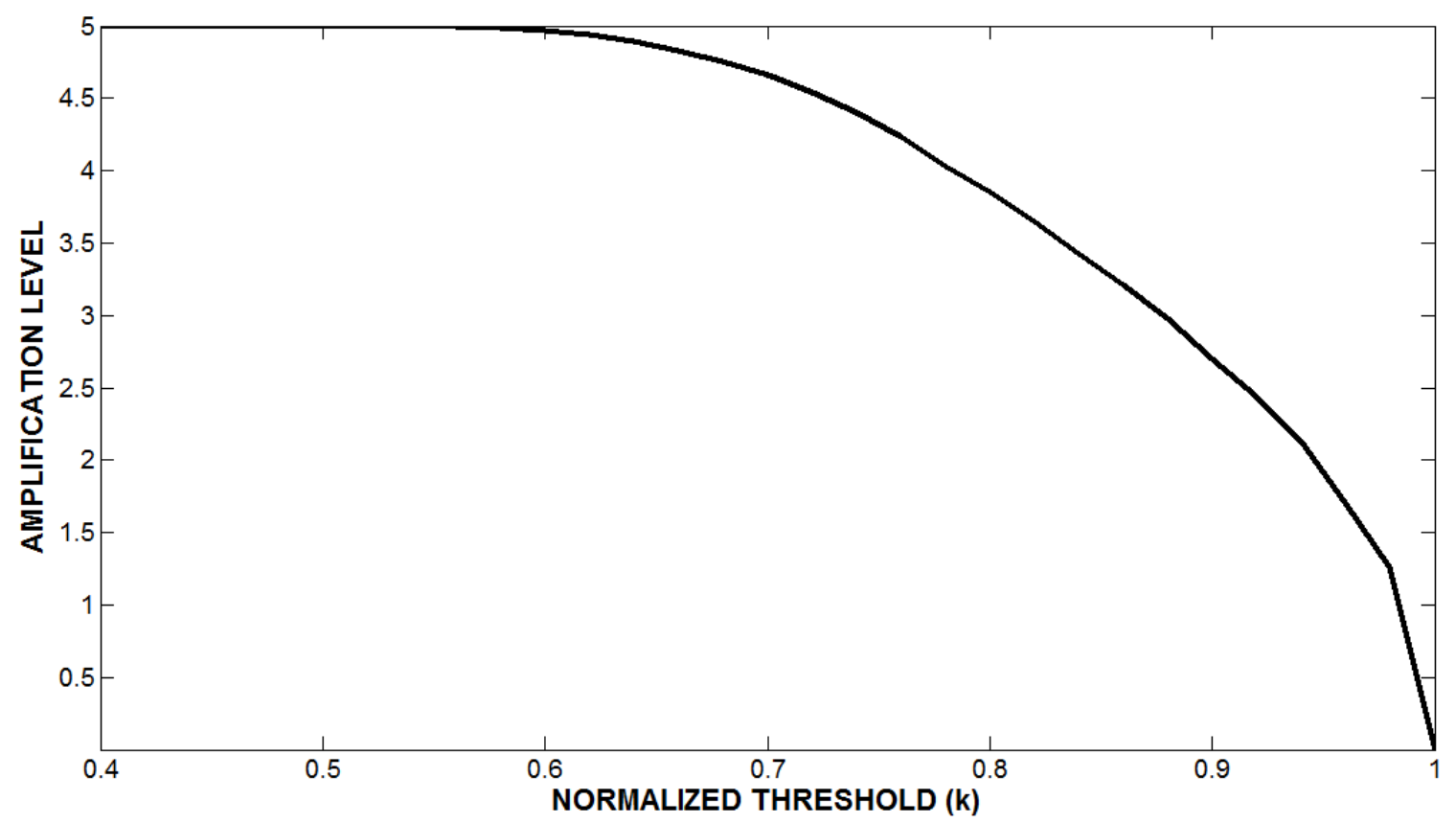

Figure 6.3: Amplification level depending on the activation threshold for a 125-node cluster of radius $2 \mu \mathrm{m}$.

number of nanomachines; in that case, due to energy-saving reasons, we should set a higher activation threshold so that the number of active nanomachines is kept to the minimum needed. The data used for this figure is extracted from the theoretical numbers of Figure 5.5 . 


\section{Chapter 7}

\section{Open Issues and Conclusions}

\subsection{Open Issues}

Nanocommunications is a really novel field, but it is quickly attracting the attention of scientists from diverse areas. Nevertheless, there is a lot of work to do and therefore many challenges appear on the way.

In this work, an analytical model for Quorum Sensing is presented and validated through simulation. The creation of novel testbeds in which the results of the simulations can be tested and validated in vivo is the next step. Experiments involving biological aspects which we are using to communicate entities at the nanoscale have to be realized, confirming theoretical and simulation results. For instance, some biological data regarding Quorum Sensing can be studied in these testbeds, such as values for the different thresholds, rates of emission, etc.

Also, several applications of Quorum Sensing for nanocommunication networks have been proposed (see Section 3.6). For instance, global synchronization, reliability or detection of complex events are examples of applications of Quorum Sensing that could be used in Wireless NanoSensor Networks. However and unlike the signal amplification case, these examples have been only outlined and need a profound analysis and the proposal of realistic schemes. The next step is to develop protocols based on these ideas to achieve synchronization, reliability or the detection of complex events in sensor networks and other types of networks. 
Regarding Quorum Sensing, many biological aspects are still relatively unknown and have to be studied in detail to fully understand this phenomenon. Indeed, after decades of research on this topic, only the surface of a really complex mechanism has been scratched. Different autoinducers, receivers, and behaviors governed by Quorum Sensing have been discovered. However, lots of more types of autoinducers, bacteria, mechanisms of Quorum Quenching, and connections between species await to be discovered and explained. A detailed study of these aspects will enable the improvement of the existing models and the extraction of realistic results.

\subsection{Conclusions}

Molecular signals suffer a significant amount of attenuation, delay and distortion as they diffuse towards the receiver. In this paper, we proposed a cooperative and bio-inspired signal amplification scheme for diffusion-based molecular nanonetworks, in order to overcome the attenuation-related limitations that these novel networks present. The methodology is based upon the biological phenomenon of Quorum Sensing, in which several randomly deployed nodes emit molecules at a constant rate and react to them by (1) emitting more molecules of the same kind and (2) executing a given command when the molecular concentration reaches a certain threshold.

By following these simple principles, synchronization between a group of nodes is accomplished so that they can jointly and coordinately transmit a certain signal, such as a pulse or a (pre)configured sequence of pulses. As the molecular channel is Linear and TimeInvariant, the received signal will be the aggregation of the contribution of each node, and therefore the signal will be effectively amplified. The level of amplification depends on the number of nodes that participate in the transmission, as shown in Figure 6.2.

The amplification level and, in turn, the final transmission range of the system will depend on the Quorum Sensing process. An analytical model of Quorum Sensing was provided in order to prove the connection between the node disposition (represented by the number of nodes and its density) and the molecular concentration in a certain volume, when 
these nodes secrete molecules in a constant rate. The expressions obtained were validated through simulation and allowed the authors to extract two essential results:

\section{Activation threshold}

Which has a direct influence to the number of nodes that will adequately activate and transmit the signal, as discussed in Section 5.3. Therefore, the transmission range that results from the amplification achieved will depend on the threshold chosen in the network dimensioning phase. Also, there exists the possibility of having a variable threshold as a function of the amplification level needed for each transmission, in order to minimize the energy consumption of the system.

\section{Synchronization Delay}

The downturn of the cooperative approach proposed in this paper is the delay that is added to the transmission. As shown in Section 5.4, the delay introduced by the synchronization process increases almost exponentially with the threshold chosen. Therefore, it is advisable to decrease the threshold in order to minimize the delay, provided that this change does not affect negatively to the quality of the transmission.

In conclusion, the results extracted from the analytical model evidence that there must be a compromise between level of amplification and delay. Low activation thresholds imply

short synchronization delays, at the expense of not being able to guarantee high levels of amplification. On the other hand, a highly set activation threshold will result in larger transmission ranges, but also in larger delays.

Cooperative Signal Amplification for Molecular Communication in Nanonetworks 


\section{Appendix A}

\section{Existing Models of Quorum Sensing}

The attention that Quorum Sensing has attracted in engineering areas is patently obvious from the quantity and variety of models and simulations that have been done. This section will serve to discuss the shortcomings of some examples, which in turn will be the reason why an automaton model is developed.

- Mathematical Models $[\mathbf{1 9}, \mathbf{3 0}, \mathbf{5 2}]$, also referred as stochastic models, these are based on the use of differential equations that describe the chemical kinetics of the reactions found inside the bacteria. The authors introduce variables for each protein and enzyme concentration, and express the change of those in terms of protein production and degradation. This chain of reactions leads to the expression of autoinducer production in terms of the intracellular and extracellular concentration, which are the catalysts of the reaction, in the end. Results are extracted from the different solutions and the stability analysis.

However, enzyme reaction chains that trigger gene regulation in Quorum Sensing vary from each species to another. Although all these different systems have been classified into three big groups (Gram-positive, Gram-negative, and hybrid; see Section 3.2), different species will end up having unique parts in their scheme apart from the common structure. For instance, in [52], a general mathematical model of Quorum 
Sensing in Gram-negative bacteria is shown, even though different species have distinct enzyme structures. Hence, specific models of Quorum Sensing for concrete species have been also developed, like in the cases of Pseudomonas aeruginosa [19] or the Agrobacterium family [30].

Another shortcoming of this approach is the lack of connection between bacteria, their enzyme reactions, and the environment. Parameters like the colony population, and factors like the interaction between bacteria or the bacterial reproduction, are the key to achieve Quorum Sensing. None of these necessary concepts is mentioned in the mathematical model of the enzyme reactions.

- Computational Models [74] simplify the chemical reactions that occur inside the bacteria, and are more centered on creating a general and macroscopic framework in which bacteria are basic elements present in an environment. This approach overcomes one of the shortcomings present in the mathematical models, in which the environment was not taken into account.

However, in [74], the bacteria are organized into clusters. Actions such as the emission of autoinducers are considered to be performed by the cluster, not by each one of the basic elements. This becomes the major shortcoming of this option, as bacteria sense the environment, emit autoinducers, and reproduce themselves as individuals. We cannot assume that all these actions are performed at the same time by all the bacteria in the colony, and in the same way.

Cooperative Signal Amplification for Molecular Communication in Nanonetworks 



\section{Bibliography}

[1] S. Abadal, "Automata Modeling of Quorum Sensing for Nanocommunication Networks," Ph.D. dissertation, Universitat Politècnica de Catalunya, 2010.

[2] S. Abadal and I. F. Akyildiz, "Bio-Inspired Synchronization for Nanocommunication Networks," To Appear in IEEE Globecom, Dec. 2011.

[3] I. F. Akyildiz, F. Brunetti, and C. Blazquez, "Nanonetworks: A new communication paradigm," Computer Networks, vol. 52, no. 12, pp. 2260-2279, 2008.

[4] I. F. Akyildiz and J. M. Jornet, "Electromagnetic Wireless Nanosensor Networks," Nano Communication Networks, vol. 1, no. 1, 2010.

[5] —_, "Electromagnetic wireless nanosensor networks," Nano Communication Networks, vol. 1, no. 1, pp. 3-19, Mar. 2010.

[6] ——, "The Internet of nano-things," Wireless Communications, IEEE, vol. 17, no. 6, pp. 58-63, 2010.

[7] M. Anissimov, "Robot Arm Places Atoms and Molecules With 100\% Accuracy," $h+$ Magazine, p. 12, 2009.

[8] B. Bassler, "How bacteria talk to each other: regulation of gene expression by quorum sensing," Current opinion in microbiology, vol. 2, no. 6, pp. 582-587, 1999.

[9] —_ "Small talk: Cell-to-cell communication in bacteria," Cell, vol. 109, pp. 421-424, 2002. 
[10] W. H. Bossert and E. O. Wilson, "The analysis of olfactory communication among animals." Journal of theoretical biology, vol. 5, no. 3, pp. 443-69, Nov. 1963.

[11] W. Bossert, "Temporal patterning in olfactory communication," Journal of Theoretical Biology, vol. 18, no. 2, pp. 157-170, 1968.

[12] C. Chen, "Development of Nanotechnology for Biomedical Applications," in Emerging Information Technology Conference. IEEE, 2005.

[13] S. Chou, P. Krauss, and P. Renstrom, "Imprint Lithography with 25-Nanometer Resolution," Science, vol. 272, no. 5258, pp. 85-87, 1996.

[14] L. C. Cobo, "Bacteria-based Communication in Nanonetworks," submitted for journal publication, 2010.

[15] S. Crosson and K. Moffat, "Photoexcited Structure of a Plant Photoreceptor Domain Reveals a Light-Driven Molecular Switch,” The plant cell, vol. 14, pp. 1067-1075, 2002.

[16] T. Danino, O. Mondragón-Palomino, L. Tsimring, and J. Hasty, "A synchronized quorum of genetic clocks," Nature, vol. 463, no. 7279, pp. 326-30, Jan. 2010.

[17] P. Devreotes, P. Derstine, and T. Steck, "Cyclic 3', 5'AMP relay in Dictyostelium discoideum," The Journal of cell biology, vol. 80, no. 2, pp. 291-299, 1979.

[18] Dictionary.com, "Dictionary.com Unabridged," 2010. [Online]. Available: http://dictionary.reference.com/browse/quorum

[19] J. D. Dockery and J. P. Keener, "A mathematical model for quorum sensing in Pseudomonas aeruginosa," Bulletin of Mathematical Biology, vol. 63, no. 1, pp. 95-116, 2001.

[20] K. M. Dolan and E. P. Greenberg, "Evidence that GroEL, not sigma 32, is involved in transcriptional regulation of the Vibrio fischeri luminescence genes in Escherichia coli." Journal of bacteriology, vol. 174, no. 15, p. 5132, 1992.

Cooperative Signal Amplification for Molecular Communication in Nanonetworks 
[21] Y. H. Dong, L. H. Wang, J. L. Xu, H. B. Zhang, X. F. Zhang, and L. H. Zhang, "Quenching quorum-sensing-dependent bacterial infection by an N-acyl homoserine lactonase," Nature, vol. 411, no. 6839, pp. 813-7, Jun. 2001.

[22] E. Drexler, C. Peterson, and G. Pergamit, "Unbounding the Future: The Nanotechnology Revolution," 1991.

[23] K. E. Drexler, Nanosystems: molecular machinery, manufacturing, and computation. New York: John Wiley \& Sons, 1992.

[24] C. Ferrier-Pages, M. Karner, and F. Rassoulzadegan, "Release of dissolved amino acids by flagellates and ciliates grazing on bacteria," Oceanologica Acta, vol. 21, no. 3, pp. 485-494, 1998.

[25] R. Freitas, "Pharmacytes: An Ideal Vehicle for Targeted Drug Delivery," Journal of Nanoscience and Nanotechnology, vol. 6, no. 9, pp. 2769-2775, 2006.

[26] C. Fuqua, S. Winans, and P. Greenberg, "Quorum Sensing in Bacteria: the LuxR-LuxI Family of Cell Density-Responsive Transcriptional Regulatorst," Journal of Bacteriology, vol. 176, no. 2, pp. 269-275, 1994.

[27] J. Garcia-ojalvo, M. B. Elowitz, and S. H. Strogatz, "Modeling a synthetic multicellular clock: Repressilators coupled by quorum sensing," Sciences-New York, vol. 101, no. 30, 2004 .

[28] N. Garralda, I. Llatser, A. Cabellos-Aparicio, and M. Pierobon, "Simulation-based Evaluation of the Diffusion-based Physical Channel in Molecular Nanonetworks," Proc. of the 1st IEEE International Workshop on Molecular and Nano Scale Communication (MoNaCom), held in conjunction with IEEE INFOCOM, 2011.

[29] H. Goldstein, "Nanotechnology: The Race to the Bottom," IEEE Spectrum, no. March $2005,2005$.

[30] A. B. Goryachev, D. J. Toh, K. B. Wee, T. Lee, H. B. Zhang, and L. H. Zhang, "Transition to quorum sensing in an agrobacterium population: A stochastic model," PLoS Computational Biology, vol. 1, no. 4, pp. 265-275, 2005.

Cooperative Signal Amplification for Molecular Communication in Nanonetworks 
[31] M. Gregori and I. F. Akyildiz, "A new NaNoNetwork architecture using flagellated bacteria and catalytic nanomotors," Journal of Selected Topics in Communications (JSAC), vol. 28, no. 4, pp. 612-619, 2010.

[32] H. Gu, J. Chao, S. Xiao, and N. Seeman, "Dynamic patterning programmed by DNA tiles captured on a DNA origami substrate," Nature Nanotechnology, vol. 4, no. April, pp. 245-248, 2009.

[33] J. Hales, S. Zheng, S. Barlow, S. Marder, and J. Perry, "Bisdioxaborine polymethines with large third-order nonlinearities for all-optical signal processing," Journal of the American Chemical Society, vol. 128, no. 35, pp. $11362-3$, Sep. 2006.

[34] J. Han, J. Fu, and R. B. Schoch, "Molecular sieving using nanofilters: Past, present and future," Lab on a Chip, vol. 8, pp. 23-33, 2008.

[35] L. Harris, L. Owens, and S. Smith, "A selective and differential medium for Vibrio harveyi," Applied and environmental microbiology, vol. 62, no. 9, pp. 3548-50, Sep. 1996.

[36] J. Henke and B. Bassler, "Bacterial social engagements," Trends in Cell Biology, vol. 14, no. 11, pp. 648-656, 2004.

[37] B. A. Hense, C. Kuttler, M. Rothballer, A. Hartmann, and J.-u. Kreft, "Does efficiency sensing unify diffusion and quorum sensing?" Nature, vol. 5, no. March, pp. 230-239, 2007.

[38] J. M. Jornet and I. F. Akyildiz, "Channel Modeling and Capacity Analysis of Electromagnetic Wireless Nanonetworks in the Terahertz Band," submitted for journal publication, April 2010, revised, April 2011.

[39] — _ "Graphene-based nano-antennas for electromagnetic nanocommunications in the terahertz band," in Proc. of 4th European Conference on Antennas and Propagation, EUCAP, Ed., Barcelona, 2010. 
[40] N. Krasnogor, M. Gheorghe, G. Terrazas, S. Diggle, P. Williams, and M. Camara, "An appealing computational mechanism drawn from bacterial quorum sensing," Bulletin of the EATCS, vol. 85, pp. 135-148, 2005.

[41] B. Lazazzera, "The intracellular function of extracellular signaling peptides." Peptides, vol. 22 , no. 10, pp. 1519-27, Oct. 2001.

[42] A. Lazdunski, I. Ventre, and J. Sturgis, "Regulatory circuits and communication in Gram-negative bacteria," Nature reviews (Microbiology), vol. 2, no. 7, pp. 581-92, 2004 .

[43] W. Leal, "Pheromone Reception," Topics In Current Chemistry, vol. 240, pp. 1-36, 2005 .

[44] H. Lee, E. Menard, N. Tassi, J. Rogers, and G. Blanchet, "Large area microcontact printing presses for plastic electronics," Materials Research Society Symposium Procedures, vol. 846, pp. 731-736, 2005.

[45] I. Llatser, E. Alarcón, and M. Pierobon, "Diffusion-based Channel Characterization in Molecular Nanonetworks," in Proc. of the 1st IEEE International Workshop on Molecular and Nano Scale Communication (MoNaCom), held in conjunction with IEEE INFOCOM, 2011.

[46] I. Llatser, I. Pascual, N. Garralda, A. Cabellos-aparicio, M. Pierobon, E. Alarcón, and J. Solé-Pareta, "Exploring the Physical Channel of Diffusion-based Molecular Communication by Simulation," Submitted for publication, 2011.

[47] M. U. Mahfuz, D. Makrakis, and H. T. Mouftah, "On the characterization of binary concentration-encoded molecular communication in nanonetworks," Nano Communication Networks, vol. 1, no. 4, pp. 289-300, Dec. 2010.

[48] C. A. Martin, A. D. Hoven, and A. M. Cook, "Therapeutic frontiers: preventing and treating infectious diseases by inhibiting bacterial quorum sensing." European journal of clinical microbiology $\&$ infectious diseases : official publication of the European Society of Clinical Microbiology, vol. 27, no. 8, pp. 635-42, Aug. 2008.

Cooperative Signal Amplification for Molecular Communication in Nanonetworks 
[49] M. Meyyappan, J. Li, and A. Cassell, "Nanotechnology: an overview and integration with MEMS," in MEMS, no. January. Istanbul, Turkey: IEEE, 2006, pp. 30-32.

[50] K. C. Mok, N. S. Wingreen, and B. Bassler, "Vibrio harveyi quorum sensing: a coincidence detector for two autoinducers controls gene expression." The EMBO journal, vol. 22, no. 4, pp. 870-81, Feb. 2003.

[51] M. Moore, A. Enomoto, and ..., "A Design of a Molecular Communication System for Nanomachines Using Molecular Motors," in Pervasive Computing and Communications Workshops. IEEE, 2006.

[52] J. Müller, C. Kuttler, B. A. Hense, M. Rothballer, and A. Hartmann, "Cell-cell communication by quorum sensing and dimension-reduction," Journal of Mathematical Biology, vol. 53, pp. 672-702, 2006.

[53] J. Murday, R. Siegel, and J. Stein, "Translational nanomedicine: status assessment and opportunities," Nanomedicine: Nanotechnology, Biology and Medicine, vol. 5, no. 3, pp. 251-273, 2009.

[54] N3Cat, "http://www.n3cat.upc.edu/n3sim."

[55] T. Nakano, T. Suda, M. Moore, R. Egashira, A. Enomoto, and K. Arima, "Molecular communication for nanomachines using intercellular calcium signaling," in Proceedings of the Fifth IEEE Conference on Nanotechnology. Nagoya, Japan: Citeseer, 2005, pp. $478-481$.

[56] T. Nakano and T. Suda, "Molecular communication through gap junction channels: System design, experiments and modeling," 2nd Bio-Inspired Models of Network, Information and Computing Systems, pp. 139-146, Dec. 2007.

[57] L. Parcerisa and I. F. Akyildiz, "Molecular Communication Options for Long Range Nanonetworks," Computer Networks, vol. 53, no. 16, pp. 2753-2766, 2009.

[58] J. Philibert, "One and a Half Century of Diffusion: Fick, Einstein, Before and Beyond," Diffusion Fundamentals, vol. 4, no. 6, pp. 1-19, 2006.

Cooperative Signal Amplification for Molecular Communication in Nanonetworks 
[59] J. Piasecki, "Centenary of Marian Smoluchowski's Theory of Brownian Motion," ACTA PHYSICA POLONICA SERIES B, vol. 38, no. 5, p. 1623, 2007.

[60] M. Pierobon and I. Akyildiz, "Diffusion-based Noise Analysis for Molecular Communication in Nanonetworks," IEEE Transactions on Signal Processing, vol. 59, no. 6, pp. 2532-47, 2011.

[61] M. Pierobon and I. F. Akyildiz, "A Physical End-to-End Model for Molecular Communication in Nanonetworks," IEEE Journal on Selected Areas in Communications (JSAC), vol. 28, no. 4, pp. 602-611, 2010.

[62] — - "Information capacity of diffusion-based molecular communication in nanonetworks," Proc. of IEEE INFOCOM Miniconference, pp. 2-6, 2011.

[63] Y. Y. Pinto, J. D. Le, N. C. Seeman, K. Musier-Forsyth, T. A. Taton, and R. A. Kiehl, "Sequence-encoded self-assembly of multiple-nanocomponent arrays by 2D DNA scaffolding," Nano letters, vol. 5, no. 12, pp. 2399-402, Dec. 2005.

[64] E. G. Ruby and L. M. Asato, "Growth and flagellation of Vibrio fischeri during initiation of the sepiolid squid light organ symbiosis," Archives of Microbiology, vol. 159, pp. 160-167, 1993.

[65] E. G. Ruby and M. J. McFall-Ngai, "A Squid That Glows in the Night: Development of an Animal-Bacterial Mutualism," Journal of Bacteriology, vol. 174, no. 15, pp. 48654870, 1992.

[66] C. Rutherglen and P. Burke, "Nanoelectromagnetics: circuit and electromagnetic properties of carbon nanotubes." Small (Weinheim an der Bergstrasse, Germany), vol. 5, no. 8, pp. 884-906, Apr. 2009.

[67] K. Salaita, Y. Wang, and C. A. Mirkin, "Applications of dip-pen nanolithography," Nature Nanotechnology, vol. 2, no. 3, pp. 145-155, 2007.

[68] F. Schedin, A. K. Geim, S. V. Morozov, E. W. Hill, P. Blake, M. I. Katsnelson, and K. S. Novoselov, "Detection of individual gas molecules adsorbed on graphene." Nature materials, vol. 6, no. 9, pp. 652-5, Sep. 2007.

Cooperative Signal Amplification for Molecular Communication in Nanonetworks 
[69] M. Schwarz, D. Romano, and M. Gheorghe, "Visualizing Bacteria Quorum Sensing," in AISB 2008 Convention Communication, Interaction and Social Intelligence, vol. 1, 2008, p. 1.

[70] J. She and J. Yeow, "Nanotechnology-enabled wireless sensor networks: From a device perspective," IEEE Sensors Journal, vol. 6, no. 5, pp. 1331-1339, 2006.

[71] R. Smith and B. Iglewski, "P. aeruginosa quorum-sensing systems and virulence," Current Opinion in Microbiology, vol. 6, no. 1, pp. 56-60, 2003.

[72] T. Suda, M. Moore, T. Nakano, R. Egashira, and A. Enomoto, "Exploratory research on molecular communication between nanomachines," in in Genetic and Evolutionary Computation Conference (GECCO). Late Breaking Papers, June, 2005.

[73] L. R. Swem, D. L. Swem, C. T. O’Loughlin, R. Gatmaitan, B. Zhao, S. M. Ulrich, and B. L. Bassler, "A quorum-sensing antagonist targets both membrane-bound and cytoplasmic receptors and controls bacterial pathogenicity." Molecular cell, vol. 35, no. 2, pp. 143-53, Jul. 2009.

[74] W. J. Tang, Q. H. Wu, and J. R. Saunders, "Individual-Based Modeling of Bacterial Foraging with Quorum Sensing in a Time-Varying Environment," Lecture Notes in Computer Science, vol. 4447, pp. 280-290, 2007.

[75] N. Taniguchi, "On the Basic Concept of 'Nano-Technology'," in Proc. Intl. Conf. Prod. Eng. Tokyo: Japan Society of Precision Engineering, 1974.

[76] D. Tessier, I. Radu, and M. Filteau, "Antimicrobial fabrics coated with nano-sized silver salt crystals," NTSI Nanotech, vol. 1, pp. 762-764, 2005.

[77] U. Ugalde, "Autoregulatory signals in Mycelial Fungi," The Mycota, vol. 1, no. 2, pp. 203-213, 2006.

[78] V. E. Wagner, D. Bushnell, L. Passador, A. I. Brooks, and B. H. Iglewski, "Microarray Analysis of Pseudomonas aeruginosa Quorum-Sensing Regulons: Effects of Growth Phase and Environment," Journal of Bacteriology, vol. 185, no. 7, pp. 2080-2095, 2003.

Cooperative Signal Amplification for Molecular Communication in Nanonetworks 
[79] K. Xavier and B. Bassler, "LuxS quorum sensing: more than just a numbers game," Current opinion in microbiology, vol. 6, no. 2, pp. 191-197, 2003.

[80] L. You, R. Cox, R. Weiss, and F. Arnold, "Programmed population control by cell-cell communication and regulated killing," Nature, vol. 428, no. 6985, pp. 868-871, 2004. 



\section{List of Figures}

1.1 Approaches for the development of nanomachines. . . . . . . . . . 8

1.2 The three steps present in diffusion-based molecular communication. . . . . 11

1.3 Molecular Communication Techniques. . . . . . . . . . . . . . . . . . 12

1.4 Molecular Communication schemes. . . . . . . . . . . . . . . . . . . 13

1.5 Alternative classification of communication options for nanonetworks. . . . 15

2.1 Reception of a pulse at distances of $10 \mu \mathrm{m}$ (solid line) and $25 \mu \mathrm{m}$ of the transmitter (dotted line). . . . . . . . . . . . . . . 20

3.1 List of autoinducers, type, chemical structure, and species that use them. . 24

3.2 Types of Quorum Sensing systems, from [36] . . . . . . . . . . . . 26

3.3 Example of parallel combination. V. harveyi case, from [36] . . . . . . . . 28

3.4 Diagram of the ventral view of an E. scolopes sample. . . . . . . . . . . . 30

3.5 Example of a collective actuation after localized sensing process. . . . . . . 37

3.6 Example of reliable sensing accomplished by means of quorum. . . . . . . 38

3.7 Example of the detection of a complex event. . . . . . . . . . . . . . 39

3.8 Example of a transmission (a) without and (b) with cooperative amplification. 41

4.1 Cubic space containing 64 nodes evenly distributed $(4 \times 4 \times 4) \ldots \ldots \ldots$

4.2 Propagation by means of diffusion of a punctual emission. . . . . . . . . 48

4.3 Block diagram of N3Sim $($ from $[46]) \ldots \ldots \ldots \ldots \ldots$

4.4 Example of a script for several iterations with different number of emitters. $\quad 59$ 
5.1 Simulation data, theoretic value and first approximation of the normalized particle concentration at a distance of $r=1 \mu \mathrm{m}$ of a constant emitter . . . .

5.2 Squared normalized distances with respect to the central node, in a perfect tridimensional grid of five units of arista. . . . . . . . . . . . 66

5.3 Central aggregation factor $\Phi(N)$ as a function of the number of nodes $N$. $\quad 68$

5.4 Position-dependent attenuation factor $\alpha$ as a function of the distance to the central node $d$ in a cluster of radius $2 \mu \mathrm{m} \ldots \ldots \ldots \ldots$

5.5 Percentage of activation of a cluster of radius $2 \mu \mathrm{m}$ as a function of the chosen threshold. . . . . . . . . . . . . . . . . . 70

5.6 Mean individual contribution factor $\Phi^{\prime}(N)=\frac{\Phi(N)}{N}$ as a function of the number of nodes $N \ldots \ldots \ldots \ldots \ldots \ldots \ldots \ldots$

5.7 Synchronization mean delay $E\left[t_{k}(d)\right]$ and standard deviation $\sigma\left[t_{k}(d)\right]$ as a function of the chosen threshold. . . . . . . . . . . . . . . 74

6.1 Reception, at a distance of $50 \mu \mathrm{m}$, of a pulse emitted by one transmitter (dotted line) and of a pulse amplified by a group of 150 transmitters (solid

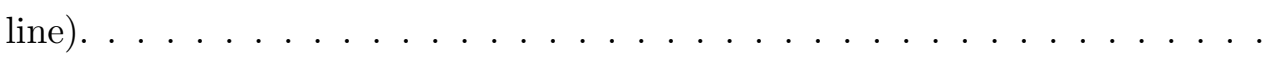

6.2 Normalized transmission range and its first order derivative as a function of the number of nodes used to amplify the signal. . . . . . . . . . . . . 78

6.3 Amplification level depending on the activation threshold for a 125-node cluster of radius $2 \mu \mathrm{m} \ldots \ldots \ldots \ldots$. . . . . . . . . . . 80 


\section{List of Tables}

2.1 Scalability of communication metrics in wireless electromagnetic and molecular channels $[45] \ldots \ldots \ldots \ldots \ldots$

3.1 Examples of functions controlled by Quorum Sensing (from [79]) . . . . . . 29

3.2 Potential therapeutic options associated to Quorum Quenching (from [48]). 35

4.1 Orientating values for Diffusion Coefficient. . . . . . . . . . . . 49

4.2 Basic parameters used in the simulator. . . . . . . . . . . . 54

4.3 Space parameters used in the simulator. . . . . . . . . . . . 55

4.4 Emitter parameters used in the simulator. . . . . . . . . . 56

4.5 Receiver parameters used in the simulator. . . . . . . . . . . 57

5.1 Summary of the notation used in the model. . . . . . . . . . . . 63

5.2 Summary of the non-elemental variables used in the model. . . . . . . . . . 72 
